\title{
Random Block Copolymer Melts in the Bulk and at Selective Substrates
}

\author{
Dissertation zur Erlangung des \\ mathematisch-naturwissenschaftlichen Doktorgrades \\ „Doctor rerum naturalium“ \\ der Georg-August-Universität Göttingen
}

\author{
vorgelegt von \\ Birger Steinmüller \\ aus Gießen
}

Göttingen 2011 
D 7

Referent: Prof. Dr. Marcus Müller Korreferent: Prof. Dr. Reiner Kree

Tag der mündlichen Prüfung: 




\section{Danksagung}

Meine Diplomarbeit in Physik handelte von der Simulation von Atomkernen. Der Wechsel aus der Kern- und Hadronenphysik ein paar Größenordnungen in Raum und Zeit hinauf in das Gebiet der weichen Materie war ein großer Schritt, aber leichter zu bewältigen, als ich erst dachte.

Der Dank dafür gebührt zuallererst Marcus Müller, der mich in seine Arbeitsgruppe aufnahm. Bei der Einarbeitung in das Gebiet der Polymerphysik und der Bearbeitung der Fragestellung dieser Doktorarbeit stand er mir mit Rat und Tat zur Seite.

Das Wissen von Kostas Daoulas über Monte Carlo Simulationen von Polymerschmelzen war entscheidend dafür, dass ich mit der Arbeit zügig beginnen konnte.

Als nächstes möchte ich mich bei meinen Zimmergenossen für die angenehme Arbeitsatmosphäre bedanken. Je remercie Fabien de m'aider à parler français et à effectuer les simulations de dynamique moléculaire. Nikita danke ich dafür, einfach Nikita zu sein. Allen anderen Gruppenmitgliedern danke ich für die schöne Zeit hier, die auch über das reine Arbeiten hinausging.

Für die Zusammenarbeit im Rahmen der Simulation von Zufallsblockkopolymeren danke ich Grant Smith, Dmitry Bedrov und Keith Hambrecht von der University of Utah.

Mein ganz besonderer Dank gebührt Martin Hömberg, für die Hilfe bei Fragen zu Algorithmen, zur Parallelprogrammierung, zur Nutzung der Rechenzentren und zu guter Letzt noch für das Korrekturlesen der Doktorarbeit.

Die Simulationen wurden in den Rechenzentren der GWDG, des HLRN und des Jülich Supercomputing Centre durchgeführt. Das Projekt wurde von der DFG im Rahmen des SPP 1369 „Polymer-Solid Contacts: Interfaces and Interphases“ finanziell gefördert.

Meinen Eltern und meiner Schwester danke ich für ihr unerschütterliches Vertrauen. 



\section{Zusammenfassung}

Zufallsblockkopolymere sind Polymere, die aus zwei verschiedenen, zufällig aneinandergefügten Typen von Blöcken bestehen. Diese Blöcke enthalten jeweils nur eine Art Monomer und eine gegenseitige Abstoßung führt hierbei zur Domänenbildung. Wenn diese Domänen unterschiedliche mechanische Eigenschaften besitzen - eine ist härter, die andere weicher - lassen sich damit beispielsweise gummiartige Werkstoffe herstellen, die ohne ein chemisches Vernetzungsmittel auskommen. Weiterhin ist die von Zufallsblockkopolymeren ausgebildete Struktur interessant für Direktmethanolbrennstoffzellen, wo diese als Protonenaustauschmembranen verwendet werden. Am Kontakt zwischen Elektroden und Membran kann sich ein ausgedehntes Gebiet bilden, das sich in seinen Eigenschaften von denen der unmittelbaren Oberfläche (Kontaktfläche) und des Volumen unterscheidet: die Interphase.

Wir verwenden für die Simulation der Polymerschmelzen zwei verschiedene vergröberte Modelle, eines mit weichen Wechselwirkungen und ein Lennard-Jones KugelFeder Modell. Im weichen Modell equilibriert die Schmelze schneller, während im Lennard-Jones Modell die Dynamik und die mechanischen Eigenschaften realistischer sind. Unser Ziel ist es, Parameter für diese Modelle zu finden, um die equilibrierten Strukturen des weichen Modells als Ausgangspunkt für das Lennard-Jones Modell verwenden zu können. Dies ermöglicht die systematische Untersuchung der mechanischen und dynamischen Eigenschaften mittels Computersimulationen. Die gefundenen Parameter führen in beiden Modellen zu Strukturen, die auf Längenskalen oberhalb weniger Segmentgrößen übereinstimmen. Die Schmelze bildet eine mikroemulsionsartige Struktur, wir sehen aber auch eine lamellare Phase. Die Verwendung von Konfigurationen des weichen Modells als Ausgangspunkt für das Lennard-Jones Modell führt zu einer Reduzierung der Equilibrierungszeit auf ein Zehntel oder weniger. Wir analysieren die mechanischen Eigenschaften der gebildeten Domänen sowie der Grenzflächen. Hier beobachten wir ein stark unterschiedliches Verhalten von Scherund Kompressionsmodul. 
In Kontakt mit einem selektiv-attraktiven Substrat bildet sich die Interphase auf bis zu vier End-zu-End Abständen heraus. Sie zeigt sich in dem Konzentrationsprofil der Monomerarten, der Fraktionierung von Kettensequenzen zwischen Interphase und Volumen, den Konformationen der Polymere und ihrer Dynamik. Der lokale Schermodul in der Interphase entsteht aus einem komplexen Zusammenspiel von Konzentration und Dichte. 


\section{Abstract}

Random block copolymers are polymers that consist of two different, randomly assembled types of blocks. Each of these blocks comprises one type of monomer and a repulsion between these monomers results in domain formation. In case that these domains have different mechanical properties - one softer, the other one stiffer - such materials may form rubbers without requiring a chemical cross-linker. The structure formed by random block copolymers is of interest for designing direct methanol fuel cells, where they are used as proton exchange membranes. There, an extended region forms at the contact area between membrane and electrodes, called the interphase, where the properties differ from those of the immediate interface and the bulk.

We use two different coarse-grained models for the simulation of the polymer melts, a Lennard-Jones bead-spring model and a model with soft interactions. The melt equilibrates faster in the soft model, while the dynamics and the mechanical properties are more accurately described by the Lennard-Jones model. We aim to find parameters for both models, so that we can use equilibrated configurations of the soft model as starting configurations of the Lennard-Jones model. This allows us to systematically investigate mechanical and dynamic properties via computer simulations. In both models, these matching parameters lead to structures which agree on length scales above a few segment lengths. We observe the formation of microemulsion-like structures as well as lamellar ones. When using equilibrated configurations of the soft model as starting configurations of the Lennard-Jones model, the equilibration time is reduced by at least a factor of ten. We analyze the mechanical properties of the domains and their interfaces and analyze the differences of the shear and the bulk modulus between the domains and the interface regions.

The contact with a selectively attractive substrate leads to the formation of an interphase which extends up to four end-to-end distances into the bulk. The interphase becomes evident in the concentration profile of monomer types, the fractionation of chain sequences between the interphase and the bulk, and the polymers' conformations and dynamics. The local shear modulus in the interphase arises from an intricate interplay between concentration and density profile. 



\section{Contents}

\begin{tabular}{lll}
\hline & Introduction & 1
\end{tabular}

$\begin{array}{lll}2 & \text { Models and Methods } & 7\end{array}$

2.1 Random Block Copolymers . . . . . . . . . . . . . . . . . . 7

2.2 Coarse-Grained Models . . . . . . . . . . . . . . . . . . . . . . . . . 11

2.3 SCMF-Simulations . . . . . . . . . . . . . . . . . 11

2.3 .1 Theoretical Background . . . . . . . . . . . . 12

2.3 .2 Simulation . . . . . . . . . . . . . . . 16

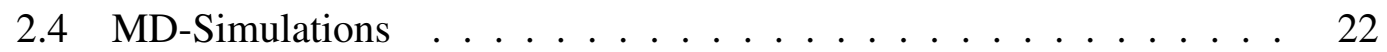

2.4 .1 Potential $\ldots \ldots \ldots \ldots \ldots$

2.4 .2 Simulation . . . . . . . . . . . . . . . . 24

\begin{tabular}{lll}
\hline & Bulk Properties & 29
\end{tabular}

3.1 Mapping in the Bulk . . . . . . . . . . . . . . . . . . 29

3.1 .1 Static Properties . . . . . . . . . . . . . 30

3.1 .2 Conformations . . . . . . . . . . . . . 36

$3.1 .3 \quad$ Dynamical Properties . . . . . . . . . . . . . . . . 38

3.1 .4 Equilibration Times After Quench . . . . . . . . . . . . . 41

3.1 .5 Equilibration After Mapping SCMF on MD . . . . . . . . . . 44

3.2 Local Mechanical Properties . . . . . . . . . . . . . . . . . . 48

$3.2 .1 \quad$ Voigt Notation and Moduli . . . . . . . . . . . . . 52

3.2 .2 Bulk Modulus . . . . . . . . . . . . . . . . . . . . 53

3.2 .3 Shear Modulus . . . . . . . . . . . . . . . . . . 54

3.3 Global Moduli . . . . . . . . . . . . . . . . . . . . . . . . . 54

3.3 .1 Glass Transition . . . . . . . . . . . . . . 54

3.3 .2 Shear and Bulk Modulus . . . . . . . . . . . . . . 55 
3.4 Local Moduli . . . . . . . . . . . . . . . . . . . . . . . . . . . . . . . 59

3.4 .1 Shear and Bulk Modulus . . . . . . . . . . . . . . . . . . . 59

3.4.2 Correlation of Local Shear Modulus and Shear Stress . . . . . 64

4 Selectively Attractive Substrate $\quad 69$

4.1 Theoretical Background . . . . . . . . . . . . . . . . . . 70

4.1 .1 Silberberg's Argument . . . . . . . . . . . . . . . . 71

4.1 .2 Linear Response Theory . . . . . . . . . . . . . . . . 73

4.2 Properties of the Interface and Interphase $\ldots \ldots \ldots \ldots$

$4.2 .1 \quad$ Wall Potential . . . . . . . . . . . . . . . . . 76

4.2 .2 Composition Profile $\ldots \ldots \ldots \ldots \ldots$

4.2 .3 Segregation at the Substrate $\ldots \ldots \ldots \ldots \ldots$

4.2 .4 Conformations at the Substrate. . . . . . . . . . . . . . . . 85

4.2 .5 Dynamics at the Substrate . . . . . . . . . . . . . . . . . 89

4.3 Mapping with a Selective Substrate . . . . . . . . . . . . . . . . 93

4.4 Mechanical Properties $\ldots \ldots \ldots$. . . . . . . . . . . . . . . . . 96

5 Conclusion and Outlook $\quad 99$ 


\section{List of Figures}

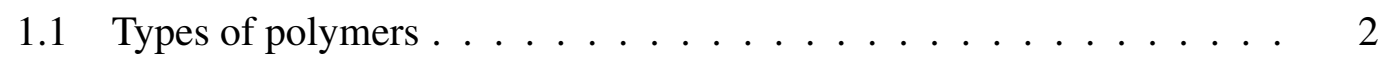

1.2 Direct Methanol Fuel Cell . . . . . . . . . . . . . . . . 3

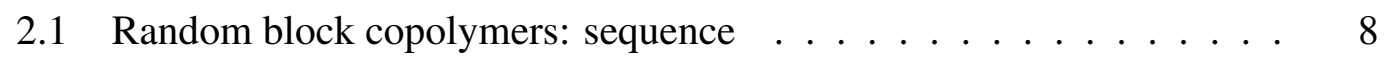

2.2 Illustration of SCMF simulations . . . . . . . . . . . . . . . . . 22

3.1 Snapshots of the bulk system $\ldots \ldots \ldots \ldots \ldots \ldots$

3.2 Comparison of $g_{A}(r)$ with $g_{B}(r) \ldots \ldots \ldots \ldots \ldots$

3.3 Comparison of $g_{A B}(r)$ and $S(q) \ldots \ldots \ldots \ldots \ldots \ldots$

3.4 Parameters of mapped structures $\ldots \ldots \ldots \ldots \ldots \ldots$

3.5 Long run SCMF $\chi_{0} N=240 \ldots \ldots \ldots \ldots \ldots \ldots \ldots$

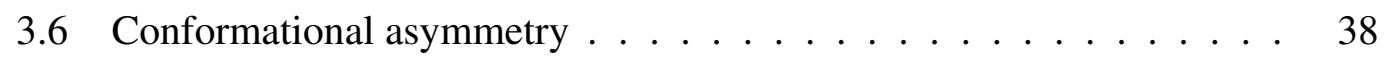

3.7 Dynamical properties, scaled $\ldots \ldots \ldots \ldots \ldots$

3.8 Dynamical properties, unscaled $\ldots \ldots \ldots \ldots \ldots \ldots$

3.9 Time evolution of $g_{A B}(r)$ and $S(q)$ in SCMF $\ldots \ldots \ldots \ldots$

3.10 Time evolution of $g_{A B}(r)$ and $S(q)$ in MD $\ldots \ldots \ldots \ldots \ldots$

3.11 Time evolution of $g_{A B}(r)$ and $S(q)$ in MD with SCMF starting config-

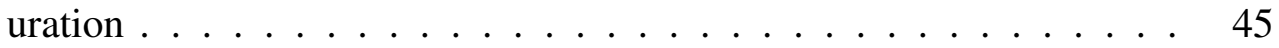

3.12 Comparison of equilibration . . . . . . . . . . . . . . 47

3.13 MSD: $k_{B} T=0.1$ and $k_{B} T=0.3 \ldots \ldots \ldots \ldots \ldots \ldots$

3.14 Shear stress: $k_{B} T=0.1$ and $k_{B} T=0.3 \ldots \ldots \ldots \ldots \ldots$

3.15 Bulk modulus: $k_{B} T=0.1$ and $k_{B} T=0.3 \ldots \ldots \ldots \ldots \ldots$. . . . 62

3.16 Shear modulus: $k_{B} T=0.1$ and $k_{B} T=0.3 \ldots \ldots \ldots \ldots . \ldots 2$

3.17 Shear stress, regions: $k_{B} T=0.3$ and $k_{B} T=0.1 \ldots \ldots \ldots$

3.18 Local shear modulus distribution . . . . . . . . . . . . . . . . 65

3.19 Stress strain locally . . . . . . . . . . . . . . . . . . . . 67

4.1 Snapshots of the substrate system $\ldots \ldots \ldots \ldots \ldots$ 
4.2 Conformations at wall . . . . . . . . . . . . . . . . . 72

4.3 Potentials of the substrates $\ldots \ldots \ldots$. . . . . . . . . . . . 77

4.4 Linear response theory: potential shape . . . . . . . . . . . . 79

4.5 Structure of melt at substrate: SCMF simulations . . . . . . . . . 81

4.6 Structure of melt at substrate: MD simulations . . . . . . . . . . . . . 82

4.7 Segregation at substrate $\ldots \ldots \ldots \ldots \ldots$

4.8 Profile of $R_{e}$ : Silberberg's argument $\ldots \ldots \ldots \ldots \ldots$

4.9 Conformations at substrate $\ldots \ldots \ldots \ldots \ldots$

4.10 MSD at substrate . . . . . . . . . . . . . . . . . . . . . . . 91

4.11 Time evolution, mapping at substrate: $\varepsilon_{A A}=1.1 \ldots \ldots$. . . . 94

4.12 Time evolution, mapping at substrate: $\varepsilon_{A A}=1.3 \ldots \ldots \ldots$. . . . 94

4.13 Time evolution, mapping at substrate: $\varepsilon_{A A}=1.6 \ldots \ldots \ldots 95$

4.14 Shear modulus profile: $\varepsilon_{A A}=1.1 \ldots \ldots \ldots \ldots$. . . . . . 97

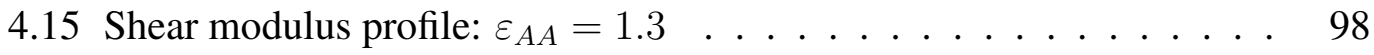

4.16 Shear modulus profile: $\varepsilon_{A A}=1.6 \ldots \ldots \ldots \ldots$ 


\section{List of Tables}

3.1 Diffusion coefficients SCMF $\ldots \ldots \ldots \ldots$

3.2 Diffusion coefficients MD $\ldots \ldots \ldots \ldots$

3.3 Bulk modulus . . . . . . . . . . . . . . . . . . . . . . . . . . 58

3.4 Shear modulus . . . . . . . . . . . . . . . . . . . 58

3.5 Density and binning: $k_{B} T=0.3$ and $k_{B} T=0.1 \ldots \ldots \ldots \ldots 61$

3.6 Bulk modulus: regions $\ldots \ldots \ldots \ldots \ldots \ldots \ldots$

3.7 Local shear modulus: $k_{B} T=0.3$ and $k_{B} T=0.1 \ldots \ldots \ldots$. . . . 64

3.8 Local shear modulus distribution . . . . . . . . . . . . . . . . 66

3.9 Correlation of local shear modulus $\ldots \ldots \ldots \ldots$

4.1 Apparent diffusivity at a selective substrate $\ldots \ldots \ldots \ldots$. . . . . 92 



\title{
1 Introduction
}

\author{
"Imagination, \\ not intelligence, \\ made us human."
}

(Terry Pratchett)

When thinking of materials used by humanity throughout its history, those which come to mind first are the ones which gave their name to ages. The stone age, in which our forefathers created a variety of tools from stone. After this, the bronze age, when human discovered how to extract copper from ore and form ever more intricate tools from its alloy bronze. Finally, there came the iron age where the use of this stronger metal was mastered.

While these three materials made the headlines, polymers have been, and continue to be even more so, important materials for usage in our society. The polymers, which exist in nature, for example fibers, wood and resins, have been in use due to their excellent and varied mechanical properties over all this time. Our prowess in understanding and creating polymeric materials has grown greatly during the twentieth century. The ubiquity of polymers in our daily lives, all the plastics that surround us, our clothes, and a plethora of composite materials, led the later Noble laureate P. J. Flory to suggest that this development has paved the way to an era "posterity may refer to as the age of polymers" [Flo73].

Polymers are macromolecules which are assembled by covalently bonding monomeric repeat units. The number of these units ranges from several hundred to thousands or even millions, and the size of the polymer greatly influences its mechanical and dynamical properties. One differentiates between homopolymers, which only consist of one type of monomeric repeat units, and copolymers, which contain two or more different monomeric repeat units. There are also a multitude of different architectures. The most simple type of polymers is the linear polymer, where the monomers form a linear chain. But there are also, to name a few, ring polymers, 


\section{Introduction}

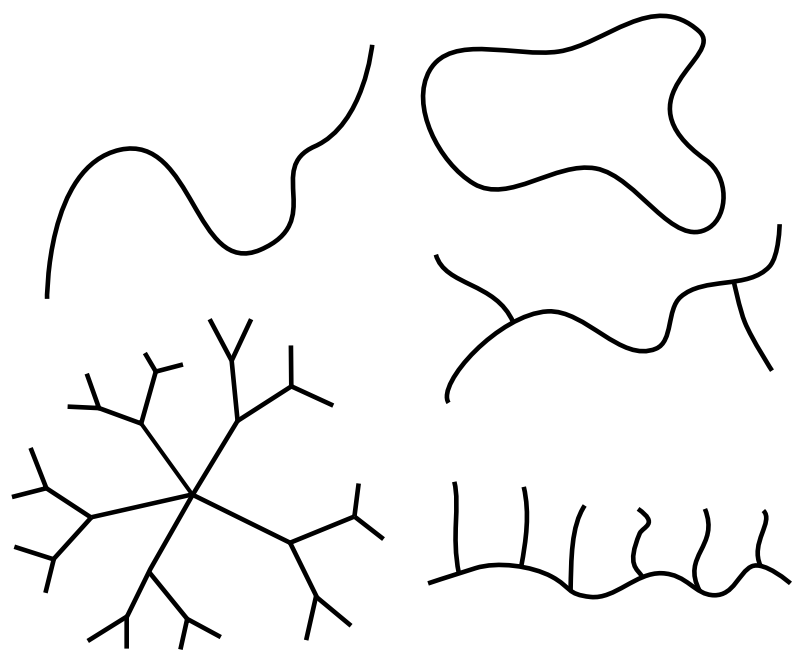

Figure 1.1: Types of polymers. These are schematic drawings of some types of polymers. They are, starting on the top left and proceeding clockwise: linear polymer, ring polymer, branched polymer, comb polymer, and dendritic polymer.

where the chain is closed, branched polymers, dendritic polymers, and comb polymers [ $\mathrm{RC} 03]$, see figure 1.1. Besides the overall structure of the polymer, the local properties, like the stiffness, play an important role in its behaviour. Furthermore, the sequence of monomers in a copolymer influences its structure formation greatly. Diblock copolymers, which consist of two homopolymer chains, comprised of different monomers, that have been joined together, have received a lot of attention due to their accessibility in theory [Lei80, MS94], simulation [YDSN94, WSBM96, MGK99] and experiment $\left[\mathrm{HHG}^{+}\right.$94, $\mathrm{TWA}^{+}$07]. A multitude of different phases have been predicted theoretically [MS94, TM05] and discovered experimentally [ $\mathrm{HHG}^{+}$94, $\mathrm{TWA}^{+}$07], depending on the length ratio of the two blocks and the interaction between the different monomers.

This thesis is, however, about random block copolymers, a special sort of multiblock copolymers where many different sequences are present in the observed system. There are two interesting areas of applicability for multiblock copolymers. Multiblock copolymers, where one component is mechanically stiffer and the other one is softer, have many uses. These include, but are not limited to biodegradable, loadbearing implants [ $\left.\mathrm{GvHS}^{+} 94\right]$, coatings for catheters, latex-free condoms and surgi- 


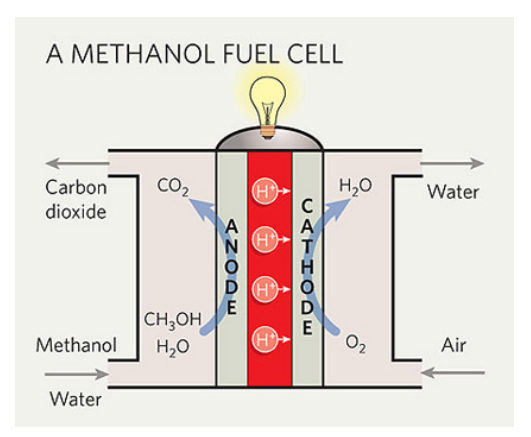

Figure 1.2: Direct Methanol Fuel Cell. This schematic shows a direct methanol fuel cell, where the proton exchange membrane is given by the red area between anode and cathode. This membrane can be made of random block copolymers, for example sulfonated poly(arylene ether sulfone) [ $\left.\mathrm{LMO}^{+} 09\right]$.

Reprinted by permission from Macmillan Publishers Ltd: Nature [Kle06], copyright 2006.

cal gloves [Yod98]. The second area of applicability lies in direct methanol fuel cells. These fuel cells operate at lower temperatures than reformed methanol fuel cells, which need these high temperatures to extract hydrogen from methanol. The methanol is in a solution of water when brought into contact with the anode. At the anode, a proton is produced and is transported through the polymeric proton exchange membrane to the cathode, where it reacts with oxygen, see figure 1.2. The problems of the currently often-used material, NAFION, [YE77], are its methanol permeability, and thermal stability. Especially a lower methanol permeability is of great interest in the research area of direct methanol fuel cells. The thermal properties of random block copolymers observed in experiments, their proton transport capability, methanol permeability, and swelling behaviour when in contact with water [HGK ${ }^{+}$04, RLM08, $\mathrm{LMO}^{+}$09, BHMW11] show great promise for this type of polymeric material to find an application in direct methanol fuel cell membranes. The properties of a fuel cell membrane are greatly dependent on the chemistry of the polymers and the charges inside it. Our simple computational model is unable to account for these properties, nonetheless, we are able to investigate the structural and mechanical properties that arise from the domain morphology.

The phase behaviour of random block copolymers has been investigated in meanfield calculations [FML92, NdlCC93, SS02] and is still subject of research today in 


\section{Introduction}

mean-field theory and numerical SCFT [vdHMZ11]. The particle-based simulation of random block copolymers has not received as much attention, due to the large system size and long relaxation times required. The number of different sequences grows exponentially with the number of blocks, therefore, the system size needed for an equal representation of every sequence becomes ever more difficult to access. There have been particle-based simulations regarding the phase diagram of random block copolymers [HM04], and more recently, an investigation of the structures random block copolymer melts form [GKKC11]. Thus, we aim to shed some more light on the properties of random block copolymer melts from a material's standpoint.

When a polymer melt is in contact with a substrate, as in the case of fuel cell membranes, where the polymer membrane is in contact with the electrodes, a new length scale, longer than the immediate interfacial region has been observed. There, the properties of the melt differ from the interface and the bulk properties. This region, called the interphase [BB95], was observed in homopolymer melts by a change in the glass transition temperature occurring in this region. When being in the lamellar phase, the lamellar ordering of a diblock copolymer melt expands far from a selectively attractive substrate, which has been observed in experiments [ $\left.\mathrm{MJR}^{+} 93\right]$. For less ordered systems, like diblock copolymer melts above the temperature of the bulk order-disorder transition [MM96] or in our case random block copolymers, an interphase region of several end-to-end radii is a possibility, in which elastic and dynamic properties differ from the bulk properties. Determining the size of this interphase region in the less well-ordered regime of random block copolymers and its elastic and dynamic properties are two of the main goals of this thesis.

In chapter 2, we start with a description of random block copolymers, and give an overview of coarse-grained models in general and, specifically, the coarse-grained models we employ. These are a soft, coarse-grained model and a Lennard-Jones beadspring model. We also introduce the simulation techniques used in conjunction with these models: Single-Chain-in-Mean-Field simulations and Molecular Dynamics.

In chapter 3, we investigate the bulk properties of random block copolymer melts. For this, we use two simulation methods, Single-Chain-in-Mean-Field simulations in conjunction with a soft, coarse-grained model, and Molecular Dynamics with a Lennard-Jones bead-spring model, to find parameters which exhibit the same mesoscopic structure. We use equilibrated configurations from SCMF simulations as starting configuration for $\mathrm{MD}$, and find that this approach drastically decreases the time 
we need to achieve equilibrated configurations in MD. In the second part of this chapter, we measure elastic properties of the melt, looking especially at the influence of a stronger incompatibility of the two monomers on the elastic properties.

The fourth chapter is devoted to the properties of random block copolymer melts in contact with a selective substrate, searching for signs of the interphase. We start with the conformations of the chains and the composition at the substrate and find that the composition close to the substrate can be predicted from the bulk structure in the case of complete wetting of one type of monomer. The process of mapping configurations of the soft, coarse-grained model to the Lennard-Jones bead-spring model is less straightforward than in the bulk, we do not find as large a decrease of simulation time needed for the equilibration of the system. We end this chapter with an investigation of the shear modulus at the substrate, where we see a sign of the interphase.

Chapter 5, contains a summary of the results and insights we have obtained, as well as an outlook at the opportunities that lie ahead in the research area of random block copolymer melts. 



\section{Models and Methods}

"If I have seen a little further it is by standing on the shoulders of Giants."

(Isaac Newton)

In this chapter we discuss the models and methods used to simulate a random block copolymer melt. We start with an introduction into the general properties of polymers, then focusing on random block copolymers. A section concerning coarse-grained models is followed by two sections, in which we discuss the simulation schemes we use. These are the Single-Chain-in-Mean-Field simulations in conjunction with a soft, coarse-grained model and molecular dynamics in conjunction with a Lennard-Jones bead-spring model. Details of the simulations, especially the numerical methods, are described in this chapter as well.

\subsection{Random Block Copolymers}

Random block copolymers are created by assembling homopolymeric blocks in a random sequence. In our simulations, we use monodisperse polymers, which means that the number of blocks, $Q$, and the block length, $m$, are held constant. In the description of random copolymers, and random block copolymers, there are two quantities which quantify the composition of the chains, $f$ and $\lambda$ [FML92]. $f_{A}=f$ is the fraction of $A$-monomers used to build the chains, and $f_{B}=1-f$ denotes the fraction of $B$ monomers. The sequence of the chains is described by the four probabilities $p_{I J}$ with $I, J \in\{A, B\}$. Each $p_{I J}$ gives the probability that a block of type $I$ is followed by a block of type $J$. There are only two independent probabilities, since

$$
\begin{aligned}
& p_{A B}=1-p_{A A} \\
& p_{B A}=1-p_{B B} .
\end{aligned}
$$




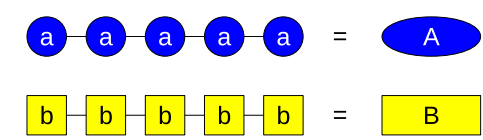

(a)

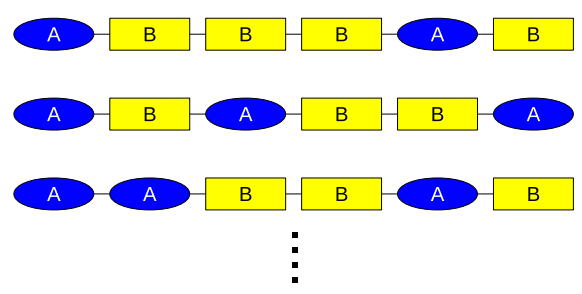

(b)

Figure 2.1: These figures show how the sequences of random block copolymers are assembled. Each block can be thought of as a prepolymer, figure 2.1(a), which are then assembled with a random sequence into random block copolymers, figure $2.1(\mathrm{~b})$.

The probabilities $p_{A A}$ and $p_{B B}$ are also constrained by the value of $f$ through

$$
f=p_{A A} f+\left(1-p_{B B}\right)(1-f)
$$

this leaves one degree of freedom which is defined as

$$
\lambda=p_{A A}+p_{B B}-1
$$

For the case of $f=\frac{1}{2}$, we can look at the extreme cases. When $\lambda=1$, the probabilities $p_{A A}$ and $p_{B B}$ have to be 1 , which results in a homopolymer blend. Choosing $\lambda=0$ results in $p_{A A}=p_{B B}=\frac{1}{2}$, thus we obtain a completely random mixture of copolymers. In the third case, $\lambda=-1, p_{A A}=p_{B B}=0$, which means that we have either one, for odd $Q$, or two, for even $Q$, types of alternating block copolymers in the melt. For the remainder of this thesis we will only consider melts with $f=\frac{1}{2}$ and $\lambda=0$.

When going about assembling the random block copolymers, two approaches can be thought of. First, we calculate the probability of each sequence. The number of sequences possible for random block copolymers consisting of $Q$ blocks is [HM04]

$$
n_{\text {sequence }}(Q)=2^{Q-1}+2^{Q_{2}-1}
$$

with $Q_{2}=\left\lceil\frac{Q}{2}\right\rceil$ signifying the next integer which is larger or equal to $\frac{Q}{2}$. The number of symmetric sequences is $2^{Q_{2}}$ and $\frac{1}{2}\left(2^{Q}-2^{Q_{2}}\right)$ is the number of asymmetric sequences. The probability of assembling a symmetric sequence is $2^{-Q}$, while it is $2^{-(Q-1)}$ for the asymmetric sequences. Now we can choose a method of the following two. In the 


\subsection{Random Block Copolymers}

first method, we choose the number of chains for our system, and, for each of these chains, randomly select a sequence according to the probabilities mentioned above. The second method always chooses multiples of $2^{Q}$ for the number of chains and the chains are not chosen randomly. We have chosen the second method, since it omits fluctuations in the composition for the benefit of having the same composition of sequences in every system.

The polydispersity index (PDI) is used to describe the mass distribution of a polymeric system. It is calculated by dividing the weight average molecular weight, $M_{w}$, by the number average molecular weight, $M_{N}$. If we take the mass of polymer $i$ as $M_{i}$, and the number of polymers of type $i$ as $N_{i}$, these quantities are calculated as follows:

$$
M_{N}=\frac{\sum_{i} N_{i} M_{i}}{\sum_{i} N_{i}}
$$

and

$$
M_{w}=\frac{\sum_{i} N_{i} M_{i}^{2}}{\sum_{i} N_{i} M_{i}}
$$

where the sum runs over all possible polymers. For our simulated system, the PDI is equal to one, for real systems it is greater or equal to one. A PDI close to one signifies that the distribution of polymer weight has a small variance. There are different methods for achieving a small value of the PDI; for multiblock copolymers, the reversible addition-fragmentation chain transfer (RAFT) polymerization is preferable over assembling these copolymers via the coupling of prepolymers [EEV10]. A possible extension of this approach is the polydispersity of the sequence. Since we use only chains of the same length, we can calculate the PDI for the number of $A$-blocks per chain. This can be done analytically, for a chain of $Q$ blocks,

$$
M_{N}=\frac{\sum_{i=0}^{Q}\left(\begin{array}{c}
Q \\
i
\end{array}\right) i}{2^{Q}}
$$

and

$$
M_{w}=\frac{\sum_{i=0}^{Q}\left(\begin{array}{c}
Q \\
i
\end{array}\right) i^{2}}{\sum_{i=1}^{Q}\left(\begin{array}{c}
Q \\
i
\end{array}\right) i}
$$

which yields an expression for the PDI as a function of $Q$

$$
\operatorname{PDI}(Q)=\frac{2^{Q} \sum_{i=0}^{Q}\left(\begin{array}{c}
Q \\
i
\end{array}\right) i^{2}}{\left[\sum_{i=0}^{Q}\left(\begin{array}{c}
Q \\
i
\end{array}\right) i\right]^{2}}=\frac{\sum_{i=0}^{Q}\left(\begin{array}{c}
Q \\
i
\end{array}\right) i^{2}\left(\frac{1}{2}\right)^{Q}}{\left[\sum_{i=0}^{Q}\left(\begin{array}{c}
Q \\
i
\end{array}\right) i\left(\frac{1}{2}\right)^{Q}\right]^{2}} .
$$




\section{Models and Methods}

These sums are known from the theory of binomial distributions, so we get, as the result for the polydispersity of the number of $A$-blocks of a random block copolymer melt

$$
\operatorname{PDI}(Q)=\frac{\frac{Q^{2}}{4}+\frac{Q}{4}}{\frac{Q^{2}}{4}}=1+\frac{1}{Q} .
$$

The phase diagram as a function of $f, \lambda$, and the incompatibility between the two types of monomers has been investigated by Fredrickson et al. [FML92] with a Landau free energy functional. For our set of parameters used to generate the random block copolymer melt, Fredrickson et al. predicted, with growing incompatibility of the two types of monomers, "regions of stability for the homogeneous phase, coexistence of two liquid phases, and disordered microphases" which lack any long-range order. Nesarikar et al. [NdlCC93] agreed with their results for $f=\frac{1}{2}$, but disagreed with their predictions for different $f$. Houdayer and Müller used the bond-fluctuation model [CK88, DB91] to determine the phase diagram of random copolymers [HM02]. They also showed that the structure of a random block copolymer melt after the phase transition can be expected to be micro-emulsion-like [HM04]. Sung and Yethiraj [SY05a, SY05b, SY05c] have studied random copolymers in the framework of integral equation theory. Their results show that no microphase separation will occur for random copolymers, [SY05a, SY05b], but they find a microphase separation for random block copolymers with a block length greater than 2 [SY05b]. Subbotin and Semenov used the weak segregation approach in the search for the type of microphases formed by a random block copolymers and found many different phases, hexagonal, bcc, and lamellar, for values of $f$ close but not equal to $\frac{1}{2}$ [SS02]. At a value of $f=\frac{1}{2}$, they predict that there is only a lamellar phase, besides the ubiquitous homogeneous phases, present in the phase diagram. More recently, there have been studies by von der Heydt et al. [vdHMZ10, vdHMZ11], using mean-field theory and numerical selfconsistent field theory to investigate the three-phase coexistence between lamellæ and the two enriched phases of microscopic phase separation. Furthermore, a study using simulations of random block copolymers through dissipative particle dynamics by Gavrilov et al. [GKKC11] probes the phase separation for very high incompatibilities of the two types of monomers, finding a lamellar phase and gyroid structures.

While the properties of random block copolymers have been investigated through a variety of methods, we want to look at them using particle-based simulations of melts with chains longer and containing more monomers than earlier investigations. We are 
interested in the structures they form, their dynamical properties, and their mechanical properties.

\subsection{Coarse-Grained Models}

For the simulation of polymers, many different models exist, each with distinctive properties. Starting at the lowest length and time scales, $n m$ and $n s$, there are quantumchemical [ $\left.\mathrm{KLM}^{+} 03\right]$ and atomistic simulations [Sun98] which simulate every atom of the molecule. Due to the large number of atoms in polymers and the intricate interactions between these atoms, the length and time resolution of these simulations is very limited. For instance, the length scale of different phases when encountering a phase transition in copolymers, or the time scale to determine properties like the diffusivity are both not within reach of these simulations. To access these properties, one resorts to coarse-grained models, where a monomer or even several monomers are represented by one effective interaction site [ $\left.\mathrm{Hel75}, \mathrm{BBD}^{+} 00, \mathrm{MP02}\right]$. It is possible in these models to access length scales of several $\mu \mathrm{m}$ and simulation times of $\mu s$. The loss of the atomistic structure, of course, generates problems - loss of friction, compressibility - which can be remedied by using sophisticated numerical methods. On the far end of the spectrum lie continuum models [MKS05]. These models, using finite element methods to simulate the system in contrast to the finite different methods employed in the aforementioned models, take the description even further away from the molecular structure. These models are often used in engineering to predict mechanical properties of macroscopic objects.

The models we employ are in the range of coarse-grained models; coarse enough to allow simulations on length and time scales where we are able to discover phase separation, but fine enough to look at the dynamics and structures of single polymers. We use two different models, one is faster while the other one keeps more detailed properties, and search for a way to incorporate the advantages of both in our investigations.

\subsection{SCMF-Simulations}

SCMF is the abbreviation of Single-Chain-in-Mean-Field simulation, a method that comprises an ensemble of single chains interacting through a mean field self-consistently generated by them [MS05, DM06]. It is based on self-consistent field theory 


\section{Models and Methods}

(SCFT), where polymers are described as flexible threads. SCFT has been used for many different systems: polymer melts [Hel75], concentrated solutions [NM02], and a variety of of polymeric structures [BF99, $\left.\mathrm{ZQZ}^{+} 10\right]$. A prominent use of SCFT is the calculation of the morphologies of diblock copolymer melts. Matsen and Schick [MS94] used SCFT to calculate the phase diagram of diblock copolymer melts. There had been phases found in experiment before, which were not in this phase diagram. Careful re-examination of these phases found them to be unstable, making the experimental phase diagram consistent with the theoretic one [Mat06]. Next to the lamellar phase, where the polymers form layers, there are the gyroid phase [ $\left.\mathrm{HHG}^{+} 94\right]$, bcc spheres, and hexagonal cylinders. This phase diagram was expanded, when another phase, called $O^{70}$, was predicted by Tyler and Morse in 2005 [TM05], even before being observed experimentally by Takenaka et al. two years later [TWA ${ }^{+}$07]. Although finite compressibility is also possible in SCFT calculations [YDSN94], SCMF simulations make it easier to describe systems with a finite incompressibility. They have been used for brushes [WM09], multi-component polymer blends [ $\mathrm{DMdP}^{+}$06], and diblock copolymer thin films [EMS ${ }^{+}$07]. The SCMF scheme incorporates fluctuations, thus the escape from local minima in the free energy is facilitated, and also enables the formation of microemulsions which is not possible in SCFT. Up to a certain extent, this also allows for the observation of dynamical properties of the system in SCMF simulations, like diffusivity and autocorrelation functions.

\subsubsection{Theoretical Background}

We start with a monodisperse homopolymer melt, using the canonical ensemble, in self-consistent field theory (SCFT). The calculations are based on [SF79, HN81, MS94, SND96]. First, we write down the partition function of the homopolymer melt

$$
\mathcal{Z} \sim \frac{1}{n !} \int \prod_{i=1}^{n} \mathcal{D}\left[\vec{r}_{i}(s)\right] \mathcal{P}\left[\Delta \vec{r}_{i}(s)\right] \exp \left(-\frac{\mathcal{H}_{n b}\left[\left\{\vec{r}_{i}(s)\right\}\right]}{k_{B} T}\right)
$$

where each of the $n$ chains is discretized into $N$ monomers. $\mathcal{D}\left[\vec{r}_{i}(s)\right]$ stands for the path integral over all possible conformation of polymer $i$, which is discretized over all monomers

$$
\int \mathcal{D}\left[\vec{r}_{i}(s)\right]=\int \prod_{s=1}^{N} d^{3} r_{i}(s)
$$


so as to sample all positions in space and conformations which the polymers can attain.

$$
\Delta \vec{r}_{i}(s)=\vec{r}_{i}(s+1)-\vec{r}_{i}(s)
$$

is the distance between two adjacent monomers, $s$ and $s+1$, of chain $i$. In this way, we can calculate the Boltzmann weight of each segment according to the bonded interaction between two monomers

$$
\mathcal{P}\left[\Delta \vec{r}_{i}(s)\right] \sim \exp \left(-\frac{\mathcal{H}_{b}\left[\Delta \vec{r}_{i}(s)\right]}{k_{B} T}\right)
$$

A harmonic potential is the usual choice for the bonded interaction. For the nonbonded interaction in the case of a homopolymer melt, we only have to enforce a homogeneous density in the melt, therefore we use an interaction which punishes deviations from the mean value.

$$
\frac{\mathcal{H}_{n b}[\hat{\phi}]}{k_{B} T}=\rho_{c} \int d^{3} r \frac{\kappa N}{2}(\hat{\phi}(\vec{r})-1)^{2}
$$

This is already done to accommodate the needs of the SCMF simulations. In SCFT, most calculations assume an incompressible melt. If this was the case here, we would use a Dirac $\delta$ function as the integrand instead of the Boltzmann weight in equation (2.11). The dimensionless density $\hat{\phi}(\vec{r})$ is calculated from the positions of monomers

$$
\hat{\phi}(\vec{r})=\frac{1}{\rho_{c}} \sum_{i=1}^{n} \sum_{s=1}^{N} \delta\left(\vec{r}-\vec{r}_{i}(s)\right)
$$

where $\rho_{c}$ is the chain density, and $\kappa N$ sets the compressibility of the system. The isothermal compressibility of the system is given by

$$
\kappa_{T}=\frac{1}{\kappa N k_{B} T \rho_{c}}
$$

see [DM06].

When we go to a more complicated system, a monodisperse homopolymer blend, we have to accommodate two different types of monomers in the non-bonded interaction and modify the form of the partition function.

$$
\mathcal{Z} \sim \frac{1}{n_{A} !} \frac{1}{n_{B} !} \int \prod_{i=1}^{n_{A}+n_{B}} \mathcal{D}\left[\vec{r}_{i}(s)\right] \mathcal{P}\left[\Delta \vec{r}_{i}(s)\right] \exp \left(-\frac{\mathcal{H}_{n b}\left[\hat{\phi}_{A}, \hat{\phi}_{B}\right]}{k_{B} T}\right)
$$




\section{Models and Methods}

Here, $n_{A}$ stands for the number of $A$-polymers and $n_{B}$ for the number of $B$-polymers. We leave the bonded interaction unchanged, but the non-bonded interaction is augmented by an additional term, proportional to $\chi_{0} N$, which characterizes the incompatibility of the different types of monomers and $\chi_{0}$ is called the Flory-Huggins parameter

$$
\frac{\mathcal{H}_{n b}\left[\hat{\phi}_{A}, \hat{\phi}_{B}\right]}{k_{B} T}=\rho_{c} \int d^{3} r \frac{\kappa_{0} N}{2}\left(\hat{\phi}_{A}(\vec{r})+\hat{\phi}_{B}(\vec{r})-1\right)^{2}-\frac{\chi_{0} N}{4}\left(\hat{\phi}_{A}(\vec{r})-\hat{\phi}_{B}(\vec{r})\right)^{2} .
$$

With this Hamiltonian, the system is completely described, but in order to calculate properties of the system, we introduce the complex, fluctuating fields $W_{A}$ and $W_{B}$, through a Hubbard-Stratonovich transform, and the collective density functions $\Phi_{A}$ and $\Phi_{B}$ through the identity

$$
\delta\left(\hat{\phi}_{A, B}-\Phi_{A, B}\right)=\int_{-i \infty}^{i \infty} \mathcal{D} W_{A, B} \exp \left(\int d^{3} r W_{A, B}\left(\Phi_{A, B}-\hat{\phi}_{A, B}\right)\right) .
$$

This does nothing to reduce the complexity of the problem, but we will use these fields later to numerically describe a polymer melt. The partition function, inserting a 'one" in the form of an integral over the functions $\Phi_{A}$ and $\Phi_{B}$ to the partition function of the homopolymer blend, equation (2.18), can then be written as

$$
\begin{gathered}
\mathcal{Z} \sim \frac{1}{n_{A} !} \frac{1}{n_{B} !} \int \mathcal{D} \Phi_{A} \mathcal{D} \Phi_{B} \prod_{i=1}^{n_{A}+n_{B}} \mathcal{D}\left[\vec{r}_{i}(s)\right] \mathcal{P}\left[\Delta \vec{r}_{i}(s)\right] \ldots \\
\ldots \delta\left(\hat{\phi}_{A}-\Phi_{A}\right) \delta\left(\hat{\phi}_{B}-\Phi_{B}\right) \exp \left(-\frac{\mathcal{H}_{n b}\left[\Phi_{A}, \Phi_{B}\right]}{k_{B} T}\right)
\end{gathered}
$$

Using the equality (2.20) transforms this equation into

$$
\begin{aligned}
\mathcal{Z} & \sim \frac{1}{n_{A} !} \frac{1}{n_{B} !} \int \mathcal{D} \Phi_{A} \mathcal{D} W_{A} \mathcal{D} \Phi_{B} \mathcal{D} W_{B} \prod_{i=1}^{n_{A}+n_{B}} \mathcal{D}\left[\vec{r}_{i}(s)\right] \mathcal{P}\left[\Delta \vec{r}_{i}(s)\right] \ldots \\
& \ldots \exp \left(-\frac{\mathcal{H}_{n b}\left[\Phi_{A}, \Phi_{B}\right]}{k_{B} T}+\int d^{3} r W_{A}\left(\Phi_{A}-\hat{\phi}_{A}\right)+\int d^{3} r W_{B}\left(\Phi_{B}-\hat{\phi}_{B}\right)\right) .
\end{aligned}
$$


Now we separate this integral into its different constituents according to their physical meaning.

$$
\begin{aligned}
\mathcal{Z} & \sim \int \mathcal{D} \Phi_{A} \mathcal{D} W_{A} \mathcal{D} \Phi_{B} \mathcal{D} W_{B} \exp \left(-\frac{\mathcal{H}_{n b}\left[\Phi_{A}, \Phi_{B}\right]}{k_{B} T}+\int d^{3} r W_{A} \Phi_{A}+W_{B} \Phi_{B}\right) \ldots \\
& \ldots \frac{1}{n_{A} !} \prod_{i=1}^{n_{A}} \mathcal{D}\left[\vec{r}_{i}(s)\right] \mathcal{P}\left[\Delta \vec{r}_{i}(s)\right] \exp \left(-\int d^{3} r W_{A} \hat{\phi}_{A}\right) \ldots \\
& \ldots \frac{1}{n_{B} !} \prod_{i=n_{A}+1}^{n_{A}+n_{B}} \mathcal{D}\left[\vec{r}_{i}(s)\right] \mathcal{P}\left[\Delta \vec{r}_{i}(s)\right] \exp \left(-\int d^{3} r W_{B} \hat{\phi}_{B}\right)
\end{aligned}
$$

The latter two integrals over the conformations of $A$ and $B$ homopolymers can be interpreted as the partition function $\mathcal{Q}_{A}^{n_{A}}$ of $n_{A}$ and $\mathcal{Q}_{B}^{n_{B}}$ of $n_{B}$ non-interacting homopolymers in an external field $W_{A}$ or $W_{B}$, respectively. At the same time

$$
\exp \left(-\frac{\mathcal{H}_{n b}\left[\Phi_{A}, \Phi_{B}\right]}{k_{B} T}+\int d^{3} r\left(W_{A} \Phi_{A}+W_{B} \Phi_{B}\right)\right)
$$

is the Boltzmann weight of a mixture of two fluids with the density functions $\Phi_{A}$ and $\Phi_{B}$ in the external fields $W_{A}$ and $W_{B}$. This re-ordering yields

$$
\begin{aligned}
\mathcal{Z} & \sim \int \mathcal{D} \Phi_{A} \mathcal{D} W_{A} \mathcal{D} \Phi_{B} \mathcal{D} W_{B} \exp \left(-\frac{\mathcal{H}_{n b}\left[\Phi_{A}, \Phi_{B}\right]}{k_{B} T}+\int d^{3} r\left(W_{A} \Phi_{A}+W_{B} \Phi_{B}\right)\right) \ldots \\
& \ldots \frac{\mathcal{Q}_{A}\left[W_{A}\right]^{n_{A}}}{n_{A} !} \frac{\mathcal{Q}_{B}\left[W_{B}\right]^{n_{B}}}{n_{B} !}
\end{aligned}
$$

We now rewrite the argument into one exponential function by using the free energy functional

$$
\begin{aligned}
\frac{F\left[\Phi_{A}, \Phi_{B}, W_{A}, W_{B}\right]}{k_{B} T} & =\frac{\mathcal{H}_{n b}\left[\Phi_{A}, \Phi_{B}\right]}{k_{B} T}-\int d^{3} r\left(W_{A} \Phi_{A}+W_{B} \Phi_{B}\right) \ldots \\
& -n_{A} \ln \left(\frac{\mathcal{Q}_{A}\left[W_{A}\right]}{V}\right)-n_{B} \ln \left(\frac{\mathcal{Q}_{B}\left[W_{B}\right]}{V}\right) \ldots \\
& +n_{A}\left(\ln \frac{n_{A}}{V}-1\right)+n_{B}\left(\ln \frac{n_{B}}{V}-1\right)
\end{aligned}
$$

and receive the final expression which now only consists of integrals over the density function and the external fields

$$
\mathcal{Z} \sim \int \mathcal{D} \Phi_{A} \mathcal{D} W_{A} \mathcal{D} \Phi_{B} \mathcal{D} W_{B} \exp \left(-\frac{F\left[\Phi_{A}, \Phi_{B}, W_{A}, W_{B}\right]}{k_{B} T}\right) .
$$

This integral cannot be evaluated, therefore it is approximated by the value at its extremum by using the saddle-point approximation [HN81], this yields the equations 


\section{Models and Methods}

which can be used to calculate the field acting on

$$
\frac{1}{k_{B} T} \frac{\delta F\left[\phi_{A}, \phi_{B}, w_{A}, w_{B}\right]}{\delta \phi_{A}}=\frac{1}{k_{B} T} \frac{\delta \mathcal{H}_{n b}\left[\phi_{A}, \phi_{B}\right]}{\delta \phi_{A}}-w_{A}=0
$$

and the density of the $A$ monomers

$$
\frac{1}{k_{B} T} \frac{\delta F\left[\phi_{A}, \phi_{B}, w_{A}, w_{B}\right]}{\delta w_{A}}=-\frac{n_{A}}{\mathcal{Q}_{A}} \frac{\delta \mathcal{Q}_{A}}{\delta w_{A}}-\phi_{A}=0
$$

The equation for the saddle point values of $w_{B}$ and $\phi_{B}$ are obtained in the same way by calculating the derivatives $F$ with respect to $\phi_{B}$ and $w_{B}$. These four equations have to be solved self-consistently, using a numerical scheme.

When turning to random block copolymers, the contribution of the single chain partition functions changes. For $Q$ blocks, there are $2^{Q-1}+1$ different block sequences.

$$
\mathcal{Z} \sim \prod_{k=1}^{2^{Q-1}+1} \frac{1}{n_{k} !} \int \prod_{i=1}^{n_{k}} \mathcal{D}\left[\vec{r}_{i}(s)\right] \mathcal{P}\left[\Delta \vec{r}_{i}(s)\right] \exp \left(-\frac{\mathcal{H}_{n b}\left[\hat{\phi}_{A}, \hat{\phi}_{B}\right]}{k_{B} T}\right)
$$

where $n_{k}$ is the number of polymers of sequence type $k$ and

$$
\begin{aligned}
\frac{F\left[\Phi_{A}, \Phi_{B}, W_{A}, W_{B}\right]}{k_{B} T} & =\frac{\mathcal{H}_{n b}\left[\Phi_{A}, \Phi_{B}\right]}{k_{B} T}-\int d^{3} r\left(W_{A} \Phi_{A}+W_{B} \Phi_{B}\right) \ldots \\
& \cdots-\sum_{k} n_{k}\left\{\ln \left(\frac{\mathcal{Q}_{k}\left[W_{A}, W_{B}\right]}{V}\right)-\left(\ln \frac{n_{k}}{V}-1\right)\right\}
\end{aligned}
$$

now depends on the single chain partition function of each of the $2^{Q-1}+1$ different polymers. We introduce an asymmetry in the non-bonded energy, namely that the two monomers' volumes differ

$$
\begin{aligned}
\frac{\mathcal{H}_{n b}\left[\hat{\phi}_{A}, \hat{\phi}_{B}\right]}{k_{B} T}= & \rho_{c} \int d^{3} r \frac{\kappa N}{2}\left((1-\alpha) \hat{\phi}_{A}(\vec{r})+(1+\alpha) \hat{\phi}_{B}(\vec{r})-1\right)^{2} \\
& -\frac{\chi_{0} N}{4}\left(\hat{\phi}_{A}(\vec{r})-\hat{\phi}_{B}(\vec{r})\right)^{2} .
\end{aligned}
$$

With this change, the energy is no longer symmetric under the exchange of $A$ and $B$ monomers.

\subsubsection{Simulation}

The method we use, Single-Chain-in-Mean-Field simulations (SCMF), incorporates fluctuations and is therefore more than a mean field solution of the equations in the 
last subsection. It is a particle-based simulation where the interaction is mediated through effective fields. The simulation volume $V$ is subdivided into cubes with an edge length of $\Delta L$. The volume fraction is calculated for each cube $m$ according to

$$
\hat{\phi}_{A, m}\left(\left\{\vec{r}_{i}(s)\right\}\right)=\sum_{i=1}^{n} \sum_{s=1}^{N} \frac{\gamma_{i}(s)}{\rho_{0} \Delta L^{3}} \Pi\left(\vec{c}_{m}, \vec{r}_{i}(s)\right)
$$

where $\rho_{0}$ is the average monomer density

$$
\rho_{0}=\frac{n N}{V}
$$

and $\gamma_{i}(s)$ is a function to determine the type of monomer $s$ of chain $i$

$$
\gamma_{i}(s)=\left\{\begin{array}{ll}
1 & \text { if the monomer is of type } A \\
0 & \text { if the monomer is of type } B
\end{array} .\right.
$$

The function $\Pi(\vec{c}, \vec{r})$ smears each monomer linearly over the eight adjacent cubes

$$
\Pi(\vec{c}, \vec{r})=\prod_{\alpha \in\{x, y, z\}} \pi\left(\left|\vec{r}_{\alpha}-\vec{c}_{\alpha}\right|\right) \quad \text { with } \quad \pi(d)=\left\{\begin{array}{ll}
1-\frac{|d|}{\Delta L} & \text { for }|d| \leq \Delta L \\
0 & \text { otherwise }
\end{array} .\right.
$$

The bonded interactions inside each chain are calculated by the following harmonic spring potential

$$
\frac{\mathcal{H}_{b}\left(\left\{\vec{r}_{i}(s)\right\}\right)}{k_{B} T}=\sum_{i=1}^{n} \sum_{s=1}^{N-1} \frac{3}{2 b^{2}} \Delta \vec{r}_{i}(s)^{2} .
$$

where $b$ is the Kuhn length of each segment and the non-interacting chain is Gaussian with an end-to-end distance of $R_{\text {eo }}^{2}=b^{2}(N-1)$.

We can now rewrite equation (2.33) into a form suitable for the grid we have defined and the non-bonded energy of the system is therefore given by

$$
\begin{aligned}
\frac{\mathcal{H}_{n b}\left[\hat{\phi}_{A}, \hat{\phi}_{B}\right]}{k_{B} T}= & \rho_{c} \Delta L^{3} \sum_{m=1}^{N_{\text {cells }}} \frac{\kappa N}{2}\left((1-\alpha) \hat{\phi}_{A, m}+(1+\alpha) \hat{\phi}_{B, m}-1\right)^{2} \\
& -\frac{\chi_{0} N}{4}\left(\hat{\phi}_{A, m}-\hat{\phi}_{B, m}\right)^{2} .
\end{aligned}
$$

The fields $w_{A}$ are calculated as in equation (2.28),

$$
\begin{aligned}
\hat{w}_{A, m} & =\frac{1}{\rho_{c} \Delta L^{3} k_{B} T} \frac{\partial \mathcal{H}_{n b}\left[\hat{\phi}_{A}, \hat{\phi}_{B}\right]}{\partial \hat{\phi}_{A, m}} \\
& =\kappa N(1-\alpha)\left((1-\alpha) \hat{\phi}_{A, m}+(1+\alpha) \hat{\phi}_{B, m}-1\right)-\frac{\chi_{0} N}{2}\left(\hat{\phi}_{A, m}-\hat{\phi}_{B, m}\right)
\end{aligned}
$$


and $w_{B}$

$$
\begin{aligned}
\hat{w}_{B, m} & =\frac{1}{\rho_{c} \Delta L^{3} k_{B} T} \frac{\partial \mathcal{H}_{n b}\left[\hat{\phi}_{A}, \hat{\phi}_{B}\right]}{\partial \hat{\phi}_{B, m}} \\
& =\kappa N(1+\alpha)\left((1-\alpha) \hat{\phi}_{A, m}+(1+\alpha) \hat{\phi}_{B, m}-1\right)+\frac{\chi_{0} N}{2}\left(\hat{\phi}_{A, m}-\hat{\phi}_{B, m}\right)
\end{aligned}
$$

as a function of the local densities $\hat{\phi}_{A}$ and $\hat{\phi}_{B}$. The fields can be used to calculate the difference in the bonded energy when the local density is changed by a small local density change, $\delta \hat{\phi}_{A}$ or $\delta \hat{\phi}_{B}$, when a monomer moves. The changes in energy are given by

$$
\frac{\mathcal{H}_{n b}\left[\hat{\phi}_{A}+\delta \hat{\phi}_{A}, \hat{\phi}_{B}\right]-\mathcal{H}_{n b}\left[\hat{\phi}_{A}, \hat{\phi}_{B}\right]}{k_{B} T}=\rho_{c} \Delta L^{3} \sum_{m=1}^{N_{\text {cell } s}} \hat{w}_{A, m} \delta \hat{\phi}_{A, m}+O\left(\delta \hat{\phi}^{2}\right)
$$

and

$$
\frac{\mathcal{H}_{n b}\left[\hat{\phi}_{A}, \hat{\phi}_{B}+\delta \hat{\phi}_{B}\right]-\mathcal{H}_{n b}\left[\hat{\phi}_{A}, \hat{\phi}_{B}\right]}{k_{B} T}=\rho_{c} \Delta L^{3} \sum_{m=1}^{N_{\text {cell } s}} \hat{w}_{B, m} \delta \hat{\phi}_{B, m}+O\left(\delta \hat{\phi}^{2}\right)
$$

respectively. With these formulæ for the total energy of the system,

$$
\mathcal{H}_{\text {total }}=\mathcal{H}_{n b}+\mathcal{H}_{b}
$$

defined in equations $(2.38)$ and $(2.39)$, we can now proceed to the simulation of the system in the canonical ensemble.

\section{Monte Carlo}

For the simulation of the system in the canonical ensemble, a smart Monte Carlo method, also called force-bias Monte Carlo, by Rossky et al. [RDF78] is used. First, we will describe the general Monte Carlo method, introduced by Metropolis et al. [MRR ${ }^{+}$53], with a focus on the particle-based system we use. When choosing a test state, we randomly move a particle, with the probability of a movement by $\vec{R}$ being constant in a cube of volume $(2 L)^{3}$ around the starting position

$$
W(\vec{R})=\Theta\left(L-R_{x}\right) \Theta\left(L+R_{x}\right) \Theta\left(L-R_{y}\right) \Theta\left(L+R_{y}\right) \Theta\left(L-R_{z}\right) \Theta\left(L+R_{z}\right) .
$$


With this probability density, we can calculate the transition probability, for the attempt to go from state $i$ to state $j$ by displacing the chosen particle by $\Delta \vec{r}_{i j}$ as

$$
T_{i j}^{*}=\frac{\int d^{3} R W(\vec{R}) \delta\left(\Delta \vec{r}_{i j}-\vec{R}\right)}{\int d^{3} \Delta r \int d^{3} R W(\vec{R}) \delta(\Delta \vec{r}-\vec{R})} .
$$

This formula might look overly complicated, however, it is also valid in case we have boundary conditions of any kind to enforce which restrict the possible positions in space of the particles. Since one wants to sample the system in the canonical ensemble, one chooses the acceptance criterion in such a way that the Markovian sequence of states approximates the canonical partition function. The Boltzmann weight of each state is given by

$$
\pi_{i}=\exp \left(-\frac{E_{i}}{k_{B} T}\right)
$$

where $E_{i}$ is the energy of the system in state $i$. The principle used for the construction of the transition probability is called detailed balance. When a system is in equilibrium, the transition from state $i$ to state $j$ has to be as likely as the transition from state $j$ to state $i$. Then the transition probability for going from state $i$ to state $j$ is given by

$$
T_{i j}= \begin{cases}T_{i j}^{*} & \text { for } \quad T_{i j}^{*} \pi_{i} \leq T_{j i}^{*} \pi_{j} \\ T_{j i}^{*} \frac{\pi_{j}}{\pi_{i}} & \text { otherwise }\end{cases}
$$

In the case of an isotropic system, the $T_{i j}^{*}$ are all equal, and the Monte Carlo method can be described in the following way:

- Choose a new position for a particle randomly inside a cube with edge length $2 L$ around it

- If the energy of the new state is lower than the initial state: accept it

- If the energy of the new state is higher, accept it with a probability $\exp \left(-\frac{E_{j}-E_{i}}{k_{B} T}\right)$

The more general transition probability for the attempted move is written as

$$
P_{M C, i j}=\min \left(1, \frac{T_{j i}^{*} \pi_{j}}{T_{i j}^{*} \pi_{i}}\right)
$$

After a move has been attempted in average for each particle, the new state is used for the calculation of canonical averages of observables. Of course, if we are working with non-equilibrated systems, they have to be equilibrated before canonical averages can be taken. 


\section{Smart Monte Carlo}

The acceptance rate of the Monte Carlo method has to be tuned with care. It can be adjusted by changing the box size from which the new position is chosen, but a too small box will hinder the simulation from covering a large amount of phase space for the canonical average in a reasonable amount of time, whereas a large box size would lead to many rejected attempts.

Rossky et al. [RDF78] introduce a method called the smart Monte Carlo method by making the acceptance of Monte Carlo moves more likely, while, at the same time, using a larger step size. The idea is based on Brownian dynamics [Erm75, AT87], using the force to choose trial displacements which are more likely to be accepted. When describing large particles in a viscous medium, the motion of these particle is governed by the Langevin equation,

$$
\ddot{\vec{r}}=-\gamma \dot{\vec{r}}+\frac{\vec{F}+\overrightarrow{\mathcal{F}}}{m}
$$

where $\gamma$ is the friction coefficient. The random force $\overrightarrow{\mathcal{F}}$ emulates the collisions of the particle with the solvent molecules. An explicit simulation of solvent molecules would take up an extraordinary amount of computation time, while at the same time contributing little to the actual behaviour of the system.

The displacement of a particle in a numerical simulation during a time step $\Delta t$ can then be written, using the Smoluchowski equation [Erm75], as

$$
\Delta \vec{r}=\frac{D \vec{F} \Delta t}{k_{B} T}+\vec{R}
$$

where $\vec{R}$ is the displacement due to the random force and $D$ is the diffusion coefficient. Taking

$$
A=D \Delta t
$$

the random displacement is chosen from a Gaussian distribution

$$
W(\vec{R})=\frac{1}{(4 A \pi)^{\frac{3}{2}}} \exp \left(-\frac{R^{2}}{4 A}\right) .
$$

In the smart Monte Carlo method, a movement of a particle by $\Delta \vec{r}_{i j}$ is attempted according to the probability

$$
T_{i j}^{*}=\frac{\int d^{3} R W(\vec{R}) \delta\left(\Delta \vec{r}_{i j}-\frac{A \vec{F}}{k_{B} T}-\vec{R}\right)}{\int d^{3} \Delta r \int d^{3} R W(\vec{R}) \delta\left(\Delta \vec{r}-\frac{A \vec{F}}{k_{B} T}-\vec{R}\right)}
$$


and accepted, with the probability

$$
P_{S M C, i j}=\min \left(1, \frac{T_{j i}^{*} \pi_{j}}{T_{i j}^{*} \pi_{i}}\right)
$$

When we choose a small value for $\Delta t$, the simulation follows Brownian dynamics. For a larger value of $\Delta t$ this breaks down, but the simulation can still be used to calculate equilibrium properties of the system. The advantage of this method is that we achieve a higher acceptance rate $P_{S M C, i j}$ than for $P_{M C, i j}$ with the same step size.

Going back to our system, we now have to write down the force to apply this method. It is simply

$$
F_{A}\left(r_{\alpha, i}(s)\right)=-\frac{1}{2 \Delta L}\left(w_{A}\left(r_{\alpha, i}(s)+\Delta L\right)-w_{A}\left(r_{\alpha, i}(s)-\Delta L\right)\right)-\frac{\partial \mathcal{H}_{b}}{\partial r_{\alpha, i}(s)}
$$

for $\alpha \in\{x, y, z\}$. For the change in energy and the force acting on the particle in the new position, there are two approaches. In one, we update the densities and use the equations (2.38) and (2.39) to determine the energy, and calculate the new fields according to the equation (2.40) or (2.41), depending on whether we attempt to move an $A$ monomer or a $B$ monomer. The results can then be used to calculate the transition probability, equation (2.55), and then randomly choose whether to accept or reject the move.

The method described in [DM06] attacks this problem from a slightly different angle. The acceptance probability in equation 2.55 can also be written as

$$
P_{S M C, i j}=\min \left(1, \frac{T_{j i}^{*}}{T_{i j}^{*}} \exp \left(-\frac{E_{j}-E_{i}}{k_{B} T}\right)\right) .
$$

We assume that the change in the local densities $\hat{\phi}_{A, m}$ and $\hat{\phi}_{B, m}$, and in the fields $\hat{w}_{A, m}$ and $\hat{w}_{B, m}$ are not large when moving one particle. Therefore, instead of the exact solution, we use the approximation of equations (2.42) and (2.43), which is accurate to first order in $\delta \hat{\phi}$, and we update the fields and densities only after the move has been accepted. The error in the second order of $\delta \hat{\phi}$ is then given by

$$
\delta E=\rho_{c}(\Delta L)^{3}\left(\kappa N-\frac{\chi_{0} N}{2}\right) \delta \hat{\phi}^{2}=\left(\kappa N-\frac{\chi_{0} N}{2}\right) \varepsilon
$$

with

$$
\delta \hat{\phi}=\frac{1}{N \rho_{c}(\Delta L)^{3}}
$$




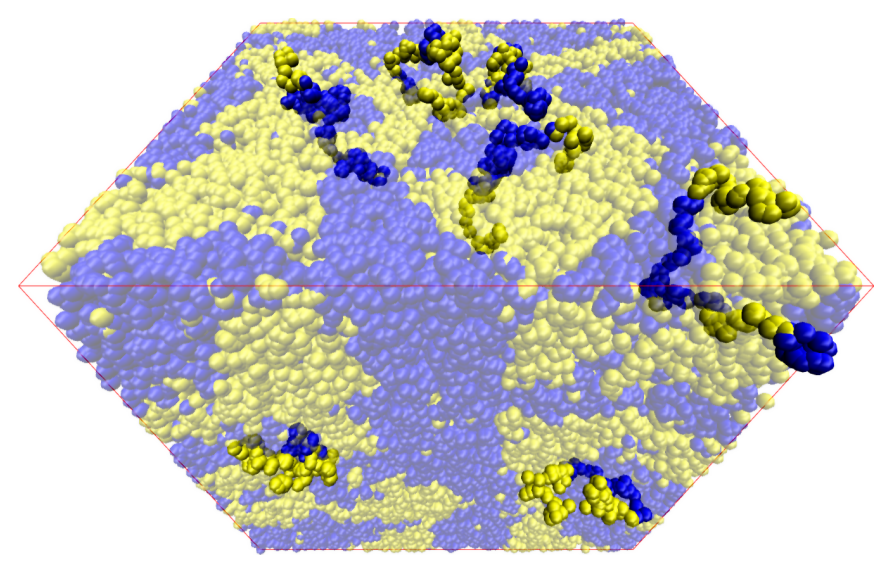

Figure 2.2: Illustration of SCMF simulations. The opaque polymer chains move in the mean field symbolized by the blurred monomers of the rest of the configuration. The chains are propagated according to the interactions with the field before the field is updated. This figure was generated using VMD[HDS96].

and therefore

$$
\varepsilon=\frac{1}{N^{2} \rho_{c}(\Delta L)^{3}}=\frac{V}{n N^{2}(\Delta L)^{3}} .
$$

Typical values of $\varepsilon$ in our simulations are $1.8 \cdot 10^{-3}$. This approach can be taken one step further, by using a method called the quasi-instantaneous field approximation [DM06]. In this case, the external fields are held constant, and are only updated after having attempted a certain number of smart Monte Carlo moves for each particle. It is paid for by a larger error, [DM06], but makes the parallelization more efficient. When one updates the densities and fields after several steps, the monomers can be distributed chainwise among processors, and communication between these processors is only necessary after the designated update period.

\subsection{MD-Simulations}

The simulation style we call MD is a combination of the way to propagate the system, Molecular Dynamics, where the equations of motion for each particle are integrated numerically, and the model used. This method was first introduced for simple liquids, for example argon [Rah64]. It took some time before the computers were powerful 
enough to access more complex macromolecules on relevant time and length scales [vGB77]. Today, molecular dynamics is a well-established simulation method in the field of polymer science [Bin95, FS02, Rap04].

\subsubsection{Potential}

The potential we use here is a 12-6 Lennard Jones potential as the non-bonded interaction and a finite extensible nonlinear elastic (FENE) potential for the bonded interactions as first proposed by Grest and Kremer in [GK86]. For the non-bonded interaction between two beads of type $I, J \in\{A, B\}$, the basic 12-6 Lennard-Jones potential has the form

$$
U_{I J, 0}(r)= \begin{cases}4 \varepsilon_{I J}\left(\left(\frac{\sigma}{r}\right)^{12}-\left(\frac{\sigma}{r}\right)^{6}\right) & \text { for } r \leq r_{c} \\ 0 & \text { for } r>r_{c}\end{cases}
$$

where $r$ is the distance between these beads, $\sigma$ is the length scale of the potential and $r_{c}$ is the cutoff distance. In the history of computer simulations, this potential was first used to describe simple liquids like Argon [Rah64]. Its repulsive part, $r^{-12}$, gives rise to a hard-core-repulsion. Together with the attractive part, $r^{-6}$, which is based on a dipole-dipole interaction, the potential's minimum is at $r_{\min }=2^{1 / 6} \sigma$ with a value of $U_{I J, 0}\left(r_{\min }\right)=-\varepsilon_{I J}$. The discontinuity of the potential energy at the cutoff $r_{c}$ is normally remediated by shifting the potential so that it is equal to zero at this point. We go a step further by eliminating not only the discontinuity in the energy, but also in the force at this point $\left[\mathrm{SRB}^{+} 07\right]$. Without a cutoff, the interaction would have to be calculated between every pair of beads, leading to an uncontrollably large computational effort for even small systems. This increase in computational effort would not be vindicated by gains in accuracy of the simulation. We use a polynomial of first order in $r$ to fix the value of the energy and the force at the cutoff

$$
U_{I J}(r)= \begin{cases}U_{I J, 0}(r)-U_{I J, 0}\left(r_{c}\right)+\left.\left(r-r_{c}\right) \frac{\partial U_{I J, 0}(r)}{\partial r}\right|_{r=r_{c}} & \text { for } r \leq r_{c} \\ 0 & \text { for } r>r_{c}\end{cases}
$$

The bonded interaction is governed by a FENE potential

$$
U_{F E N E}(r)= \begin{cases}-\frac{1}{2} k r_{0}^{2} \ln \left(1-\frac{r^{2}}{r_{0}^{2}}\right) & \text { for } r \leq r_{0} \\ \infty & \text { for } r>r_{0}\end{cases}
$$

The value of $r_{0}$ is set to $1.5 \sigma$ [GK86]. 


\subsubsection{Simulation}

For the simulation of the polymers in the Kremer-Grest model, we use the MD simulation package LAMMPS [Pli95]. In MD simulations, the equations of motion are solved numerically to propagate the system. The LAMMPS code uses spatial decomposition methods for parallelization. In spatial decomposition, the simulation volume is divided into subvolumes, whose contents are distributed to different processors. Each subvolume is then simulated on its own processor. Of course, the processors have to communicate the interactions on particles inside the cutoff distance of their boundary, as well as when particles cross these boundaries. Depending on the ensemble one wants to use, however, these equations differ. We will now describe the ensembles, which will later be used to simulate the systems and the basic method used for the solution of the equations of motion.

\section{NVE}

The first ensemble we mention, although it is the most seldom used one in this work, is the microcanonical ensemble. In the microcanonical ensemble, the particle number, the volume and the energy are held constant, therefore the equations of motion to be solved for the velocity and the momentum are

$$
\begin{aligned}
\dot{\vec{r}_{i}} & =\frac{\vec{p}_{i}}{m} \\
\dot{\vec{p}}_{i} & =\vec{F}_{i} .
\end{aligned}
$$

\section{NVT}

The second ensemble is the canonical one, with the Helmholtz free energy as the thermodynamic potential. In the canonical ensemble, the particle number, the volume and the temperature are held constant. The temperature, as a collective quantity, cannot be simply fixed to a certain value. To control the temperature, one needs to introduce a thermostat [Hoo85].

$$
\begin{aligned}
\dot{\vec{r}}_{i} & =\frac{\vec{p}_{i}}{m} \\
\dot{\vec{p}}_{i} & =\vec{F}_{i}+\zeta \vec{p}_{i} \\
\dot{\zeta} & =\nu_{T}^{2}\left(\frac{T(t)}{T_{\text {ext }}}-1\right)
\end{aligned}
$$


where $T(t)=\frac{2}{X k_{B}} \sum \frac{\vec{p}^{2}}{2 m}$, with $\mathrm{X}$ being the number of the system's degrees of freedom. Hoover showed in his paper that these equations of motion are equivalent to the canonical ensemble, regardless of the values of the parameters. In the form we use, from [MCH93], it uses an update frequency $\nu_{T}$, to force the temperature back to its set value $T_{\text {ext }}$. Hoover used a different form for the last equation

$$
\dot{\zeta}=\frac{1}{Q}\left(\sum \frac{\vec{p}^{2}}{m}-X k_{B} T\right)
$$

where $Q$ can be thought of as the mass of the thermostat. However, selecting the value of the mass, or the update frequency, influences the dynamics of the system. For a low value of the update frequency, it takes a long time for the system to return back to the desired temperature, which means that taking a canonical average takes a much longer time. When $\nu_{T} \rightarrow 0$, the equations of motions become the microcanonical ones. A very high frequency leads to strong temperature oscillations, which are especially evident when changing the temperature of the system [ [FS02].

\section{NPT}

In the isobaric-isothermal ensemble, with the Gibbs free energy as the thermodynamic potential, the particle number, pressure and temperature are constant. Thus, we need a thermostat [Hoo85] and a barostat [Hoo86] to control the intensive quantities temperature and pressure, respectively. The equations of motion by Hoover were improved by Melchionna et al. [MCH93], and are as follows

$$
\begin{aligned}
\dot{\vec{r}}_{i} & =\frac{\vec{p}_{i}}{m}+\eta\left(\vec{r}_{i}-\vec{R}_{0}\right) \\
\dot{\vec{p}}_{i} & =\vec{F}_{i}+(\eta+\zeta) \vec{p}_{i} \\
\dot{\zeta} & =\nu_{T}^{2}\left(\frac{T(t)}{T_{e x t}}-1\right) \\
\dot{\eta} & =\frac{\nu_{P}^{2}}{N k_{B} T_{e x t}} V\left(P(t)-P_{e x t}\right) \\
\dot{V} & =d V \eta .
\end{aligned}
$$

The pressure $P(t)$ is calculated by

$$
P(t)=\frac{N k_{B} T}{V}+\frac{\sum_{i<j}\left(\vec{r}_{i}-\vec{r}_{j}\right) \cdot \vec{F}_{i j}}{d V}
$$




\section{Models and Methods}

with the sum running over all pairs of particles, and $\vec{F}_{i j}$ the force between two particles. The differences to the equations of motion in the canonical ensemble, see equation (2.65), govern the pressure $P$, and consequently, the simulation volume size $V$. The pressure the system should have is expressed by $P_{e x t}$, and there is, as for the temperature, an update frequency, $\nu_{P}$. Since the volume is not constant anymore, its change follows equation (2.69), where $d$ is the number of dimensions of the simulated system. When the volume changes, the positions of the particles have to be rescaled as well. This is done in equation 2.67, with the center of mass $\vec{R}_{0}$. Again, as for the canonical ensemble, the update frequencies $\nu_{T}$ and $\nu_{P}$ can be set to any non-zero value for the system to sample the isobaric-isothermal ensemble. However, the same restriction that were mentioned with regard to choosing the update frequency of the temperature applies to the update frequency of the pressure as well, it should neither be too big nor too small to yield a result in reasonable time.

\section{$\mathrm{NP}_{\mathrm{t}} \mathrm{T}$}

For a system which is on both sides constrained by an immovable substrate, we use an anisotropic isobaric-isothermal ensemble. While the temperature is thermostated regardless of the dimension, the pressure is only barostated tangentially. The equations of motion in $x$-direction

$$
\begin{aligned}
\dot{r}_{x, i} & =\frac{p_{x, i}}{m} \\
\dot{p}_{x, i} & =F_{x, i}+\zeta p_{x, i}
\end{aligned}
$$

and in $y / z$-direction

$$
\begin{aligned}
\dot{r}_{y / z, i} & =\frac{p_{y / z, i}}{m}+\eta\left(r_{y / z, i}-R_{y / z, 0}\right) \\
\dot{p}_{y / z, i} & =F_{y / z, i}+(\eta+\zeta) p_{y / z, i} \\
\dot{\eta} & =\frac{\nu_{p}^{2}}{N k_{B} T_{\text {ext }}} A\left(P(t)-P_{\text {ext }}\right) \\
\dot{A} & =d A \eta
\end{aligned}
$$

are connected through the thermostat

$$
\dot{\zeta}=\nu_{T}^{2}\left(\frac{T(t)}{T_{\text {ext }}}-1\right) .
$$

This type of ensemble is also used for the equilibration of membranes, where one is interested in a tensionless state. This is achieved by setting $P_{\text {ext }}$ to zero [HM10]. 


\section{NEMD}

The aforementioned methods describe simulations done in equilibrium. When we look at mechanical properties of the system by deforming it, we need to apply nonequilibrium MD (NEMD). Tuckerman et al. [TMBK97] developed a method called SLLOD, based on the DOLLS Hamiltonian for a fluid under shear, see Hoover et al. $\left[\mathrm{HEH}^{+} 80\right]$. The equations of motion are for a system at constant volume and constant temperature, therefore a thermostat is used again, as in equation (2.65).

$$
\begin{aligned}
\dot{\vec{r}}_{i} & =\frac{\vec{p}_{i}}{m}+\vec{r}_{i} \vec{\nabla} \cdot \vec{u} \\
\dot{\vec{p}}_{i} & =\vec{F}_{i}-\vec{p}_{i} \vec{\nabla} \cdot \vec{u}+\zeta \vec{p}_{i} \\
\dot{\zeta} & =\nu_{T}^{2}\left(\frac{T(t)}{T_{\text {ext }}}-1\right) .
\end{aligned}
$$

The quantity $\vec{u}$ is a flow field introduced in the fluid.

\section{Velocity Verlet}

The velocity Verlet algorithm is used for the integration of the $N V E$ equations of motion (2.64). It derives its name from a method by Verlet [Ver67], used for the microcanonical ensemble. The equations used today are better suited for numerical computations. Swope et al. [SABW82] showed them to be equivalent to Verlet's integration scheme and Tuckerman et al. brought them into their currently used form [TBM92]. It is equivalent to using a Adams-Moulton method for the integration of the velocities and a Taylor expansion for the integration of the position. Both of these numerical schemes are accurate to second order in $\Delta t$.

The method calculates the velocities of the particles at an intermediate step. It does not have to store these velocities for further use.

$$
\begin{aligned}
\dot{\vec{r}}_{i}\left(\frac{\Delta t}{2}\right) & =\dot{\vec{r}}_{i}(0)+\frac{\Delta t}{2 m} \vec{F}_{i}(0) \\
\vec{r}_{i}(\Delta t) & =\vec{r}_{i}(0)+\Delta t \dot{\vec{r}}_{i}\left(\frac{\Delta t}{2}\right) \\
\dot{\vec{r}}_{i}(\Delta t) & =\dot{\vec{r}}_{i}\left(\frac{\Delta t}{2}\right)+\frac{\Delta t}{2 m} \vec{F}_{i}(\Delta t)
\end{aligned}
$$





\title{
3 Bulk Properties
}

\author{
"There is always a \\ well-known solution to \\ every human problem - \\ neat, plausible, and wrong." \\ (H. L. Mencken)
}

Studying a polymeric system in simulations, we start with the bulk properties in this chapter.

In the first section, which has been accepted as a paper [SMH$\left.{ }^{+} 11\right]$, we explore static properties, like the radial pair correlation function, the static structure factor of the composition, and the conformations of the different types of polymers. We show that we can find parameters in the two different models, the soft, coarse-grained model and the Lennard-Jones bead-spring model, for which the static properties coincide. In this section we also elaborate on dynamical properties and compare the time needed to equilibrate the system. We propose a mapping scheme to use SCMF configurations as starting points for MD simulations.

The remainder of the chapter is devoted to the calculation of mechanical properties with the help of MD simulations. It starts with a section describing the methods used to calculate the shear and bulk moduli of the systems. These methods are applied in the following two sections. First, to describe the bulk properties of the system, and then to access the mechanical properties on a more local level.

\subsection{Mapping in the Bulk}

There have been many efforts to map the parameters of different models, either to find a way to map bidirectionally between two models $\left[\mathrm{SRB}^{+} 07\right]$ or for using multiscale simulations [HK08, PdSK08, VB09], where one switches between different 


\section{Bulk Properties}

models during a single simulation run, or even between different domains of one simulation volume. There are also intricate schemes to equilibrate polymeric melts faster by carefully selecting the conformations of the polymers' starting configuration [AEG ${ }^{+} 03$, HM08]. These methods, while being very efficient, are only needed for longer chains than the ones we use. They are needed for highly entangled melts, since entanglement is one of the properties the soft, coarse-grained model is unable to reproduce. For the chains we study, mapping $N=120$ from the soft, coarse-grained model to $N=60$ in the Lennard-Jones bead-spring model, there are so few entanglements per chain that a less intricate approach is sufficient.

\subsubsection{Static Properties}

The snapshots shown in figure 3.1 illustrate how the morphology of the melt changes with growing incompatibility of the two different monomeric repeat units. To tune the incompatibility of the monomers in the soft, coarse-grained model, we use $\chi_{0} \mathrm{~N}$ and the asymmetry parameter $\alpha$. In the Lennard-Jones bead-spring model, we vary only one parameter, $\varepsilon_{A A}$. Already for a small incompatibility in both models, see panels 3.1(a) and 3.1(d), $A$ and $B$ segments are not randomly mixed but they show a preference to form clusters, albeit with small differences in composition. The internal interfaces of these clusters or concentration fluctuations of one type of segment are rather broad.

For the next set of parameters, $\varepsilon_{A A}=1.3$ and $\chi_{0} N=100$, a microemulsion-like structure has formed, cf. panels 3.1(b) and 3.1(e), where the domains are segregated more strongly and the interfaces can now be clearly determined.

In the final set of parameters, depicted in panels 3.1(c) and 3.1(f), the microemulsionlike structure has fully formed, and therefore the internal interfaces between the domains are rather sharp. The morphology is characterized by a well-defined characteristic length scale but no long-range order like in a lamellar phase is established.

Although these snapshots are generated by simulating different models, where there is no analytical expression to identify matching parameters, they show a striking similarity in their mesoscopic structure in a statistical sense. The first goal of our study is to identify parameters of the two different models to produce this structural similarity.

We chose $R_{\text {melt }}$, the root of the mean squared end-to-end distance of a homopolymer in a melt, as the length scale, which allows us to compare the results of these two models. The values are $R_{\text {melt }}=1.2 R_{\text {eо }}$ in the soft, coarse-grained model used in 


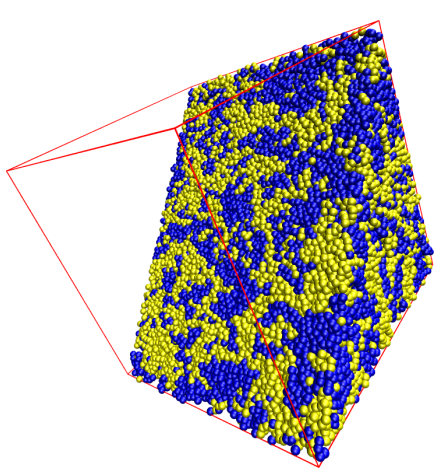

(a) $\varepsilon_{A A}=1.1$

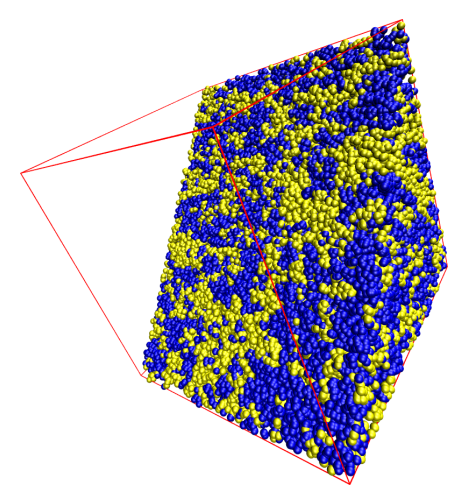

(d) $\chi_{0} N=30$

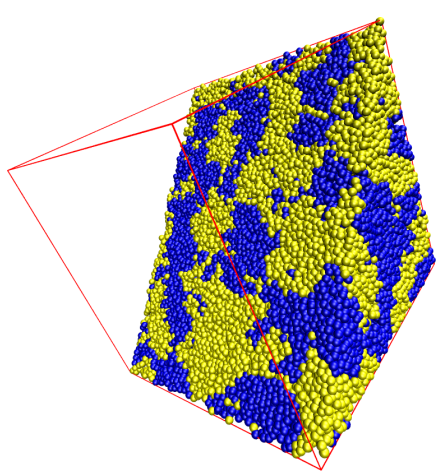

(b) $\varepsilon_{A A}=1.3$

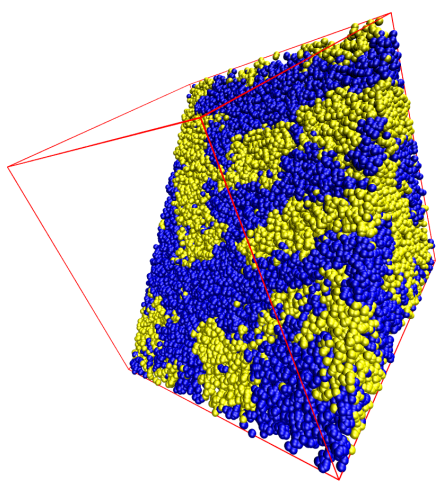

(e) $\chi_{0} N=100$

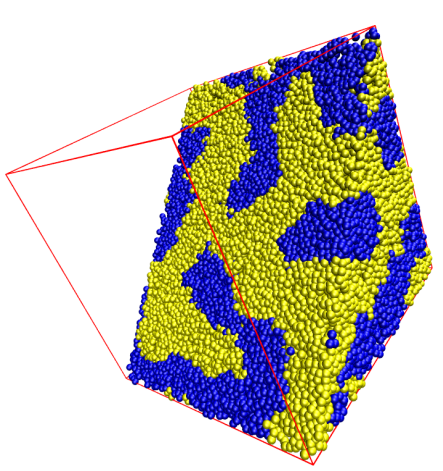

(c) $\varepsilon_{A A}=1.6$

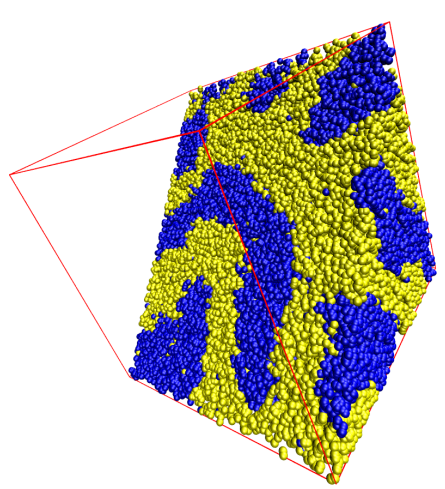

(f) $\chi_{0} N=240$

Figure 3.1: Snapshots for different values of the interaction parameter as indicated below each figure. On the top, the snapshots are from MD simulations while the bottom figures show snapshots obtained by SCMF simulations. These figures were produced using VMD[HDS96].

the SCMF simulation and $R_{\text {melt }}=10.0 \sigma$ in the MD simulation, respectively. The quantities, which we used to characterize the static structure, are the static structure factor of the composition, $S(q)$, as well as the radial pair correlation function of $A$ and $B$ segments, $g_{A B}(r)$. The structure factor is defined as

$$
S(q)=\frac{1}{n N}\left\langle\left|\sum_{i=1}^{n} \sum_{s=1}^{N}\left(2 \gamma_{i}(s)-1\right) \exp \left(i \vec{q} \vec{r}_{i}(s)\right)\right|^{2}\right\rangle .
$$

with $\gamma_{i}(s)$ as in equation (2.36). The choice of values for $q$ is constrained by our finite simulation box. Since it is cubic, we can only calculate $S(q)$ for values of $q=\frac{2 \pi}{L} \sqrt{k_{x}^{2}+k_{y}^{2}+k_{z}^{2}}$ where all $k_{i}$ are integers. The $A-B$ pair correlation function 


\section{Bulk Properties}

is defined as

$$
g_{A B}(r)=\frac{\int d^{3} r_{1} \int d^{3} r_{2}\left\langle\rho_{A}\left(\vec{r}_{1}\right) \rho_{B}\left(\vec{r}_{2}\right)\right\rangle \delta\left(r-\left|\vec{r}_{1}-\vec{r}_{2}\right|\right)}{\int d^{3} r_{1} \int d^{3} r_{2}\left\langle\rho_{A}\left(\vec{r}_{1}\right)\right\rangle\left\langle\rho_{B}\left(\vec{r}_{2}\right)\right\rangle \frac{4 \pi r^{2}}{V}}
$$

where $\rho_{A, B}(\vec{r})$ are the local densities.

The time scale of the structural relaxation of the single-chain conformations and the morphology is measured in units of the time $\tau$ that it takes a center of mass of a homopolymer, $\chi_{0} N=0$ or $\varepsilon_{A A}=1.0$, in a homopolymer melt of the same chain length and number of chains to diffuse a distance on the order of $R_{\text {melt }}^{2} ; \tau=R_{\text {melt }}^{2} / D$, where $D$ denotes the self-diffusion coefficient in a homopolymer melt and $R_{\text {melt }}^{2}$ is the measured mean squared end-to-end distance of polymers in a homopolymer melt. In the SCMF simulations we obtain $\tau=2.75 \cdot 10^{5}$ SMC steps, where each segment on average had the chance of one trial displacement in a SMC step. In the MD simulations we obtain $\tau=2.1 \cdot 10^{7}$ integration steps.

In order to match the equilibrium structure of the soft, coarse-grained model to the Lennard-Jones bead-spring model, we have adjusted the parameters incompatibility, $\chi_{0} N$, and the segmental asymmetry, $\alpha$, keeping the inverse compressibility, $\kappa N=600$ fixed. Varying $\alpha$ does not influence the $A B$ pair correlation function, but the $A A$ and $B B$ pair correlation functions. By varying this parameter we change the local density in $A$ - and $B$-rich domains to better reflect the asymmetry of the Lennard-Jones beadspring model. This change of the density of $A$ and $B$ rich domains is depicted in figure 3.2 . It becomes very pronounced for higher values of the incompatibility in the Lennard-Jones bead-spring model and this change is reproduced by the change of $\alpha$. When one changes the value of $\chi_{0} N$, it results in a shift of position and change of magnitude of the peak in the pair correlation function. A greater value of $\chi_{0} N$ shifts this peak to larger length scales and increases its height.

In figure 3.3 the pair correlation functions are plotted for three sets of parameters, which show similar structure on a mesoscopic length scale. The analogous data for the structure factor are displayed in the insets.

Due to the harsh repulsion of the Lennard-Jones bead-spring model, as compared to the soft interactions of the soft, coarse-grained model which allow segments to overlap, the two structural properties of both models cannot be reconciled on short length scales for the pair correlation function and large $q$-values for the structure factor, respectively. This difference manifests itself as a peak of the structure factor for MD simulations around $\left(q R_{\text {melt }}\right)^{2}=5000$, which corresponds to $(q \sigma)^{2}=50$, and the packing structure 


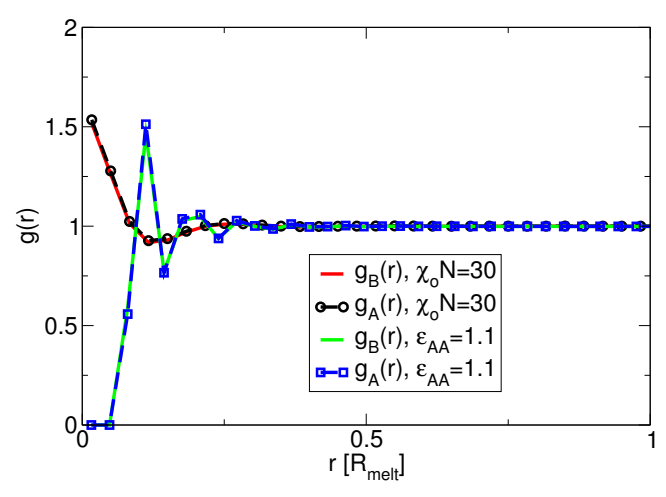

(a) $\varepsilon_{A A}=1.1$

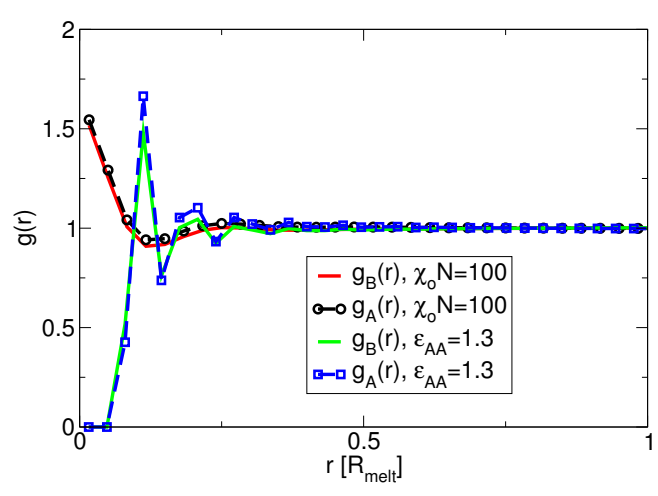

(b) $\varepsilon_{A A}=1.3$

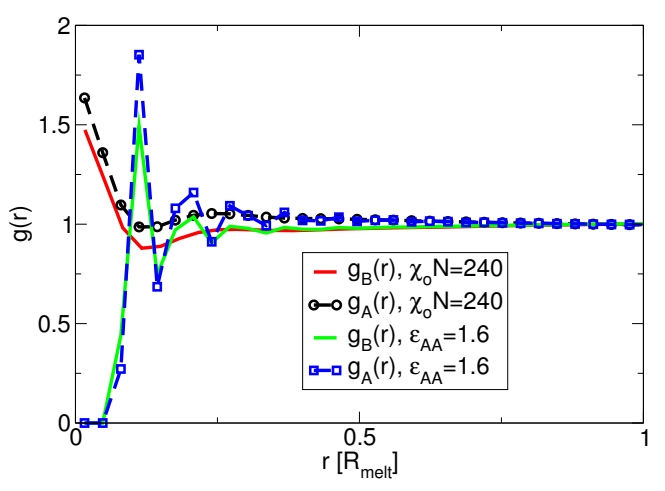

(c) $\varepsilon_{A A}=1.6$

Figure 3.2: Comparison of $g_{A A}(r)+g_{B B}(r)$. The radial pair correlation function of $A\left(g_{A}=\left(g_{A A}+g_{A B}\right) / 2\right)$ and $B\left(g_{B}=\left(g_{B B}+g_{A B}\right) / 2\right)$ monomers are compared for three different sets of parameters in the two different models. For $r>0.5 \cdot R_{\text {melt }}$, the pair correlation function of $A$ is greater than the one of $B$ at higher incompatibility. This is an indication of a higher density of A.rich domains than of $B$-rich domains in accordance with our model parameters. As parameters in the SCMF simulations, $\kappa N$ was always set to $600, \alpha=0.01$ and $\chi_{0} N=30$ in 3.2(a), $\alpha=0.02$ and $\chi_{0} N=100$ in 3.2(b), $\alpha=0.05$ and $\chi N=240$ in $3.2(\mathrm{c})$.

of the pair correlation function. At intermediate to large length scales, however, the equilibrium structure agrees very well for each set of parameters. Let us discuss the three different sets in turn. For the smallest incompatibilities, $\chi_{0} N=30$ and $\varepsilon_{A A}=$ 1.1, we observe a correlation hole in the $A B$ pair correlation function, figure $3.3(\mathrm{a})$, 


\section{Bulk Properties}

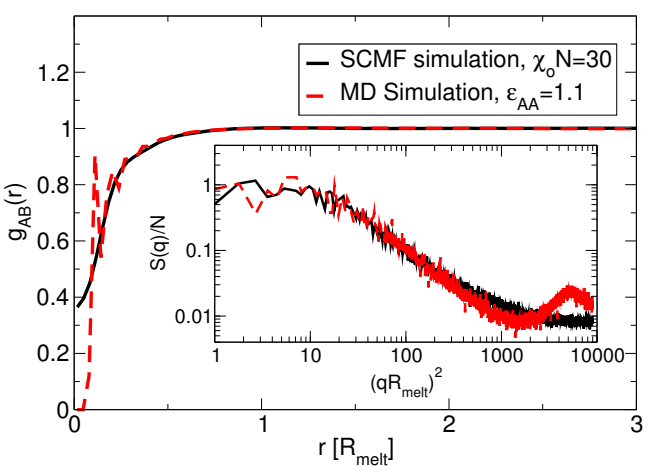

(a) $\varepsilon_{A A}=1.1$

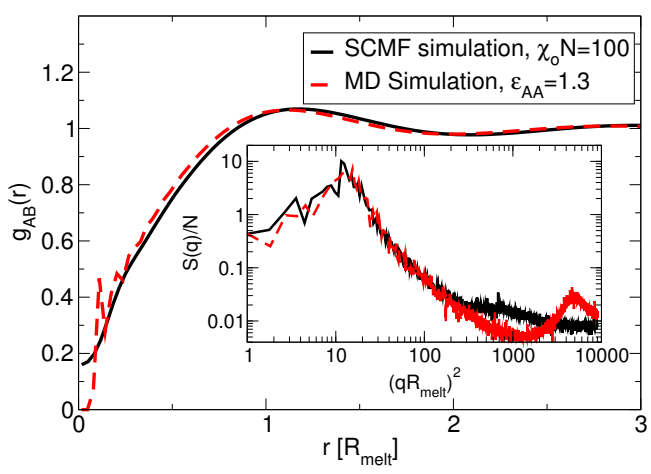

(b) $\varepsilon_{A A}=1.3$

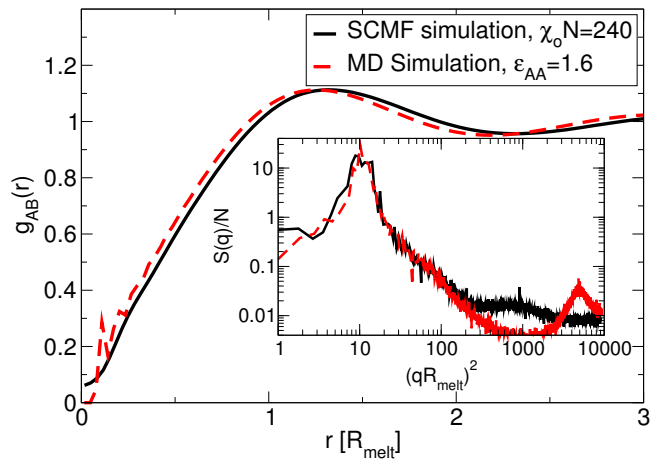

(c) $\varepsilon_{A A}=1.6$

Figure 3.3: Comparison of the radial A-B pair correlation function and the structure factor for three different sets of parameters. As parameters in the SCMF simulations, $\kappa N$ was always set to $600, \alpha=0.01$ and $\chi_{0} N=30$ in 3.3(a). $\alpha=0.02$ and $\chi_{0} N=100$ in 3.3(b); $\alpha=0.05$ and $\chi_{0} N=240$ in 3.3(c).

but no peak appears, neither in the pair correlation function, nor in the structure factor, which is displayed in the inset. As the snapshots have already suggested, this value of interaction strength fails to impose a strong segregation onto the system. When we increase the interaction strength to $\chi_{0} N=100$ and $\varepsilon_{A A}=1.3$, see figure 3.3(b), we observe a formation of a structure on a scale of $1.2 R_{\text {melt }}$ in the pair correlation function and a peak in the structure factor at $\left(q R_{\text {melt }}\right)^{2} \approx 14$, which corresponds to a wavelength of $1.7 R_{\text {melt }}$. The position of this peak of the structure factor corresponds to a point of inflection in the pair correlation function. Increasing the interaction strength further to $\chi_{0} N=240$ and $\varepsilon_{A A}=1.6$, cf. figure 3.3(c), the peak of the pair correlation function shifts to $1.3 R_{\text {melt }}$ and the peak in the structure factor to $\left(q R_{\text {melt }}\right)^{2} \approx 11$. Again, the 
position of this peak in the structure factor, with a wavelength of $1.9 R_{\text {melt }}$, and the point of inflection of the pair correlation function coincide.

Mean-field theory predicts that at intermediate segregation, random block copolymers form a microphase separated morphology with a characteristic wavelength that decreases with increasing incompatibility [FM91], as $r_{d} \sim\left(\chi-\chi_{\text {crit }}\right)^{-0.5}$. This prediction differs from our simulation results. First, the morphology, which we observe in the simulation, lacks long-range order. Second, the characteristic length scale increases as we increase the incompatibility between unlike segments. We summarize the results of this subsection in figure 3.4 plotting the pairs of $\left(\chi_{0} N, \alpha\right)$ and $\varepsilon_{A A}$, for which we found good agreement of the static structure at mesoscopic length scales. From now on, when referring to the parameters used in SCMF simulations, only mentioning the value of $\chi_{0} N$ will be synonymous to the pair of values as given in figure 3.4 .

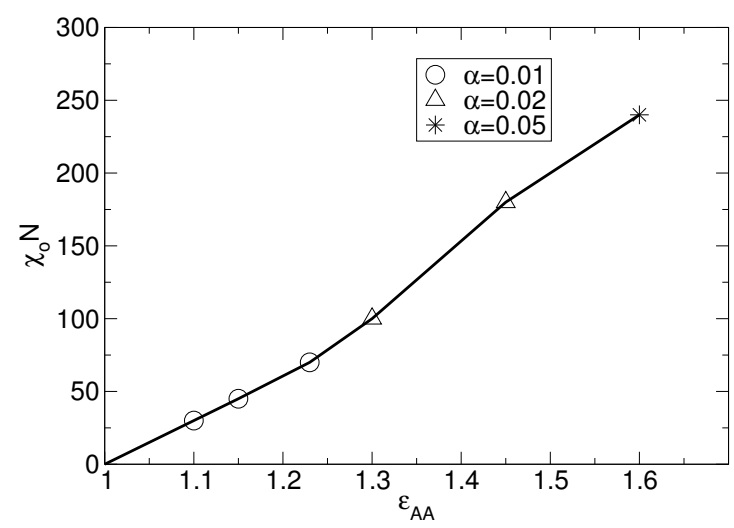

Figure 3.4: Parameters of mapped structures. In this figure we show the parameters we found to give similar static structures in the two different models. In the SCMF simulations, $\kappa N=600, \alpha=0.01$ for $30 \leq \chi_{0} N \leq 75, \alpha=0.02$ for $100 \leq \chi_{0} N \leq 180$ and $\alpha=0.05$ for $\chi_{0} N=240$.

\section{Lamellar Phase}

For very long runs, $t>50 \tau$, and the highest incompatibility, $\chi_{0} N=240$, we have observed a change in the structure factor. At this large time scale, which take time in SCMF simulations but are hardly accessible in MD due to the even slower dynamics at $\varepsilon_{A A}=1.6$, see tables 3.2 and 3.1 , a single peak develops in the structure factor, see 


\section{Bulk Properties}

figure 3.5(a) Since we want to compare the structure formation of MD with SCMF, we have to stay in the regime which is accessible to both models. However, this is an indication that the prediction of a lamellar phase, as in [SS02], holds. A snapshot of this configuration is shown in in figure $3.5(\mathrm{~b})$. This result is also in accordance with the extensive study of random block copolymer morphologies in a high segregation regime by Gavrilov et al. [GKKC11].

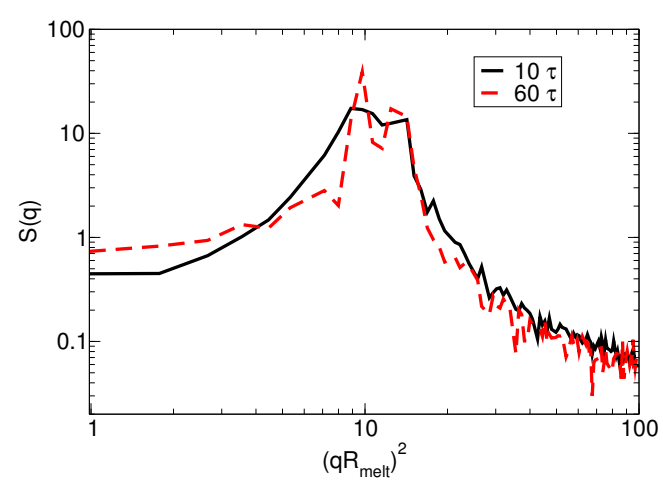

(a)

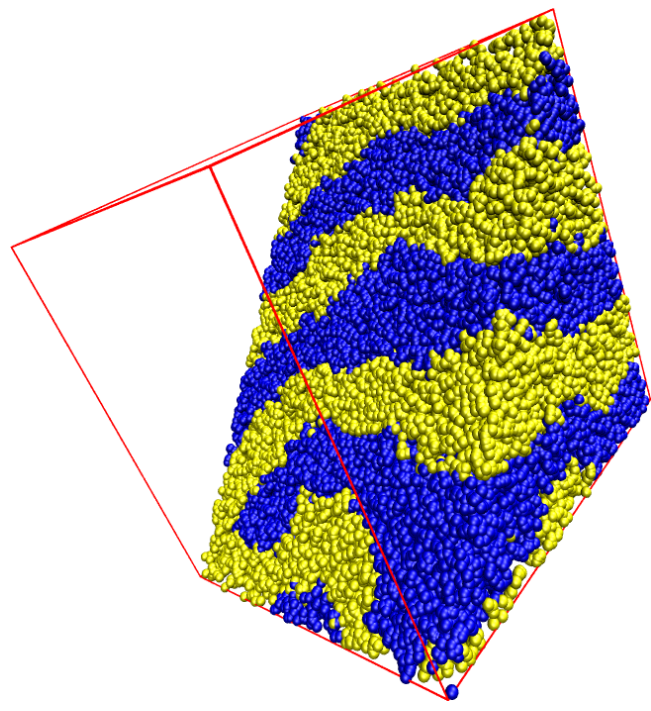

(b)

Figure 3.5: Long run SCMF $\chi_{0} N=240$. For a long simulation of the random block copolymer melt, $t \sim 60 \tau$, we discover a microphase separation into a lamellar structure. The left figure shows the structure factor with a clearly discernible peak at $\left(q R_{\text {melt }}\right)^{2}=9.7$ while the snapshot on the right shows the lamellar structure.

\subsubsection{Conformations}

In the previous subsection we have demonstrated that one can identify parameters for the morphology of the soft, coarse-grained model and the Lennard-Jones beadspring model at intermediate and long length scales to coincide. In this subsection we investigate to what extent this structural agreement holds for conformational properties of individual molecules as well. 
Chain conformations have been analyzed by examining the mean squared radius of gyration, $R_{\mathrm{g}}^{2}$,

$$
R_{\mathrm{g}}^{2}=\sum_{i=1}^{N} \sum_{s=1}^{n}\left\langle\left(\vec{r}_{i}(s)-\vec{r}_{i, \mathrm{com}}\right)^{2}\right\rangle
$$

where $\vec{r}_{i, \text { com }}$ is the coordinate of the center of mass of polymer $i$, and the mean squared end-to-end distance, $R_{\mathrm{e}}^{2}$. In this analysis we have sorted all chains based on the overall chain composition. Since all chains consist of 6 blocks there are 7 different types of chains with $0,1,2,3,4,5$ or 6 blocks of type $B$. Conformational properties were averaged for each chain composition. Figure 3.6 shows the change of conformations as a function of the interaction parameters, $\varepsilon_{A A}$ and $\chi_{0} N$, for different chain composition. As the interaction strength increases, the conformations of chains with different composition show dissimilarities. With the formation of a microemulsion-like structure, homopolymer chains become more compact as is evident from the reduced values of $R_{\mathrm{g}}^{2}$ in figure 3.6(a), compared to the homopolymer melts with $\varepsilon_{A A}=1.0$ and $\chi_{0} N=0$. The shrinking of the molecular extension is likely a consequence of the confinement into corresponding $A$ or $B$ domains. Interestingly, after a well-segregated morphology forms, $\varepsilon_{A A} \geq 1.3$ and $\chi_{0} N \geq 100$, the conformations of homopolymer chains are almost independent of the strength of the interaction and the corresponding changes in structure observed in figure 3.3 .

Chains that contain both $A$ and $B$ blocks become more extended, compared to a homopolymer melt, as the interaction strength increases. This molecular stretching is the strongest for chains with 50-50 composition. We also calculated the ratio of $\left\langle R_{\mathrm{e}}^{2}\right\rangle /\left\langle R_{\mathrm{g}}^{2}\right\rangle$ to examine if the stretching or the shrinking of chains affects their Gaussianity. For Gaussian chains this ratio is 6 , [ [RC03]. Figure 3.6(b) shows that chains with 50-50 A/B composition deviate strongly from the Gaussian behaviour with their ratio being larger than 6.0 as a sign for extended conformations. Chains with 33 or $67 \%$ of $A$ segments have ratios very close to 6 indicating that, on average, their conformations are similar to a Gaussian chain. Finally, homopolymer chains ( $A$ or $B$ ) and chains with large composition asymmetry have $\left\langle R_{\mathrm{e}}^{2}\right\rangle /\left\langle R_{\mathrm{g}}^{2}\right\rangle$ ratio smaller than 6.0 indicating a more coiled conformations compared to Gaussian chains.

We emphasize that the same parameters that result in an agreement of the morphology between the Lennard-Jones bead-spring model and the soft, coarse-grained model additionally give rise to good agreement of the conformational properties. 


\section{Bulk Properties}

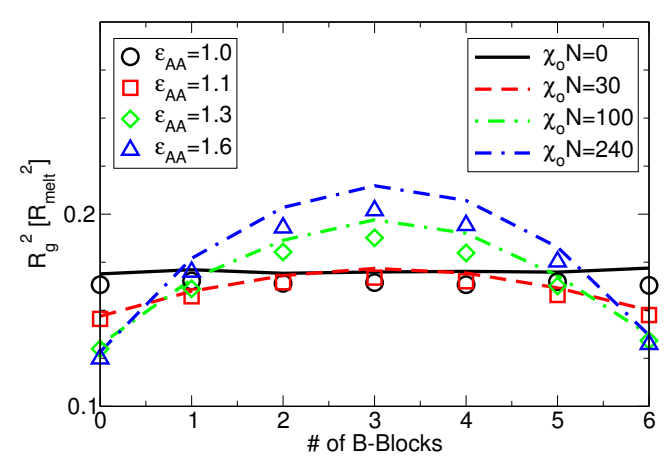

(a) $R_{g}^{2}$

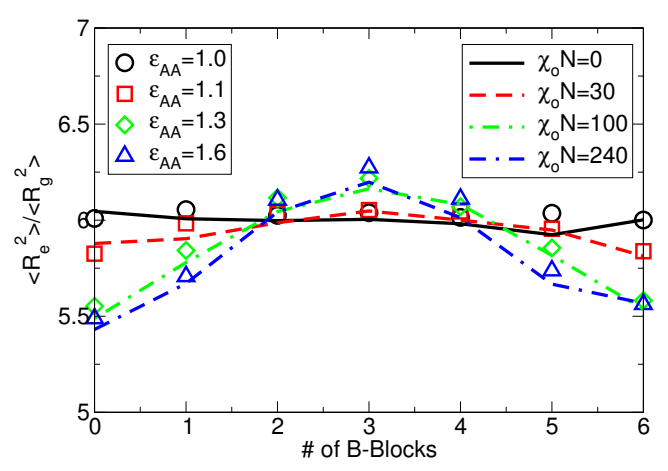

(b) $R_{e}^{2} / R_{g}^{2}$

Figure 3.6: Conformational asymmetry. In these figures, the conformational properties of the two different models are examined as a function of interaction strength and the number of B blocks of the chains. The mean squared radius of gyration, $R_{g}^{2}$, normalized by the mean squared end-to-end distance of the homopolymer melt, is shown in the left figure, 3.6(a), to investigate the extension of the polymers. The figure to the right, 3.6(b) is used to examine the Gaussianity of the chains by plotting $R_{g}^{2} / R_{e}^{2}$. Both figures show these results for SCMF and MD simulations

\subsubsection{Dynamical Properties}

In this subsection, we compare the single-chain dynamics of the soft, coarse-grained model and the Lennard-Jones bead-spring model. In figure 3.7, we present the mean squared displacement (MSD), and the end-to-end vector autocorrelation

$$
C(t)=\left\langle\frac{\vec{R}_{e}(t) \cdot \vec{R}_{e}(0)}{R_{e}(t) R_{e}(0)}\right\rangle
$$

as a function of time. The time scale is adjusted by the diffusion coefficient, $D$, see tables 3.1 and 3.2 . The diffusion coefficient is calculated from the MSD of the polymers' centers of mass,

$$
g_{3}(t)=\sum_{i=1}^{N}\left\langle\left(\vec{r}_{i, c o m}(t)-\vec{r}_{i, c o m}(0)\right)^{2}\right\rangle
$$

[PBHK91], by $D=\lim _{t \rightarrow \infty} \frac{g_{3}(t)}{6 t}$. The diffusion coefficient decreases by a factor of two when we compare the disordered melt, $\chi_{0} N=0$, 30, with the micro-emulsion like, 


\begin{tabular}{|l|l|l|l|l|}
\hline$\chi_{0} N$ & 0 & 30 & 100 & 240 \\
\hline$D / D_{\text {melt }}$ & 1.0 & 0.88 & 0.49 & 0.46 \\
\hline
\end{tabular}

Table 3.1: Diffusion coefficients SCMF. The diffusion coefficients of the polymer chains in SCMF simulations are calculated for different parameters of the soft, coarse-grained model

\begin{tabular}{|l|l|l|l|l|}
\hline$\varepsilon_{A A}$ & 1.0 & 1.1 & 1.3 & 1.6 \\
\hline$D / D_{\text {melt }}$ & 1.0 & 0.83 & 0.25 & 0.11 \\
\hline
\end{tabular}

Table 3.2: Diffusion coefficients MD. The diffusion coefficients of the polymer chains in MD simulations are calculated for different parameters of the LennardJones bead-spring model

$\chi_{0} N=100,240$, systems. We can deduce that the formed structure severely restricts the mobility of the chains. When we compare the MSD for a single segment, the $A$ segments show a slightly smaller value for large length scales, see figures $3.7(\mathrm{a})$ and 3.7(b).

The end-to-end vector autocorrelation function in figure 3.7(d) shows that the time needed for the end-to-end vector to decorrelate undergoes a larger change than the diffusion coefficient. This time grows by a factor of 5 when we compare $\chi_{0} N=30$ to $\chi_{0} N=100$, and a factor of 10 when we compare $\chi_{0} N=30$ to $\chi_{0} N=240$. The influence of the formation of domains and their boundaries becomes more pronounced in this quantity, while also being noticeable in the MSD.

When looking at the same quantities, MSD and the end-to-end vector autocorrelation function without adjusting the time scale by the diffusion coefficients, see figure 3.8, the dynamical quantities show a significantly different behaviour as a function of the interaction strength. We examine the MSD of single segments in figures $3.8(\mathrm{a})$ and 3.8(b) for $A$ - and $B$-segments, respectively. For short times, an increase of the interaction strength does not affect the motion of $B$-segments, their freedom becomes constricted only at larger time scales, when the collective motion of the entire chains dominates. For $A$-segments, a larger interaction strength also gives rise to a reduction of mobility at short time scales in the MD simulations. The difference in the MSD of single segments decreases with longer time scales. In figure $3.8(\mathrm{~d})$, we investigate the autocorrelation function of the polymers' end-to-end vector. As in the case of the 


\section{Bulk Properties}

MSD, this dynamical quantity implies the same slower dynamics of the MD melt when compared to the SCMF model.

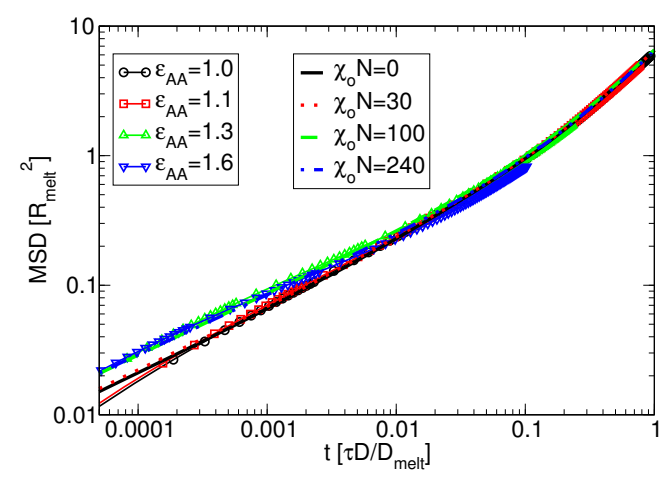

(a) MSD A-segments

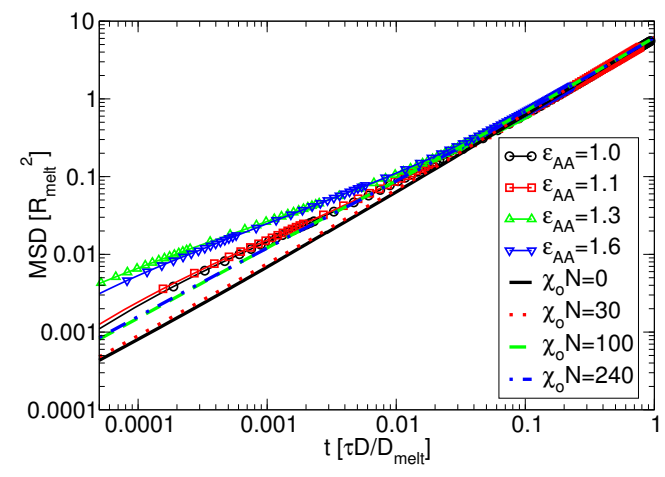

(c) MSD centers of mass

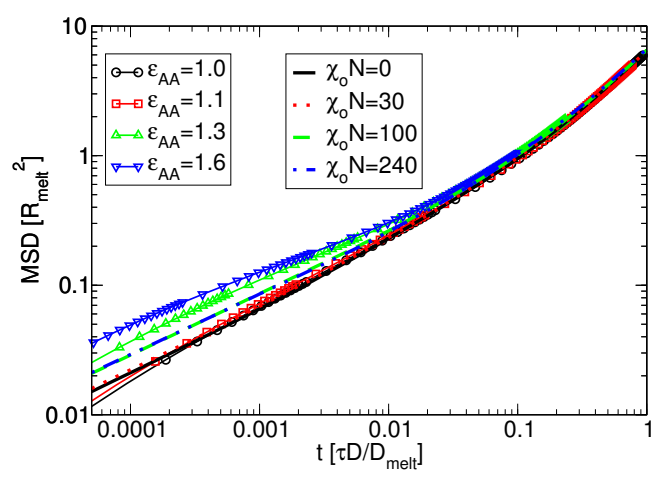

(b) MSD B-segments

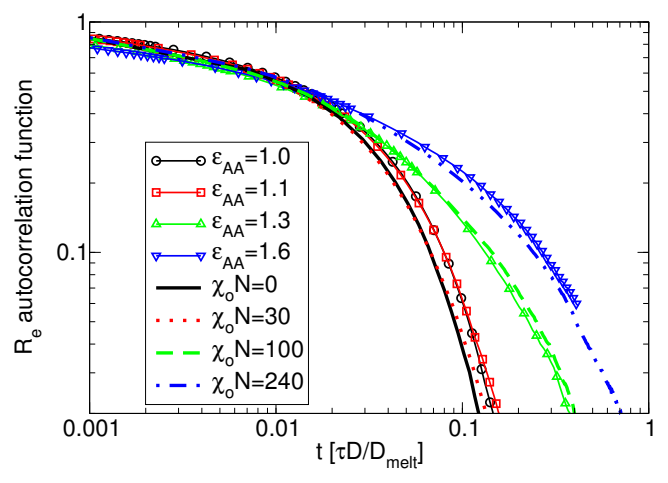

(d) $R_{e}$-acf

Figure 3.7: Dynamical properties, scaled. Here we show some dynamical properties of our SCMF-systems, namely the MSD of 3.7(a) A-segments and 3.7(b) Bsegments. In $3.7(\mathrm{~d})$ the end-to-end vector autocorrelation function demonstrates the different time scales of these systems. The time is multiplied with the diffusion coefficient of the random block copolymer melt and divided by the diffusion coefficient of the homopolymer melt. The end-toend vector autocorrelation function decorrelates on the same time scale for MD and SCMF for each set of parameters. 


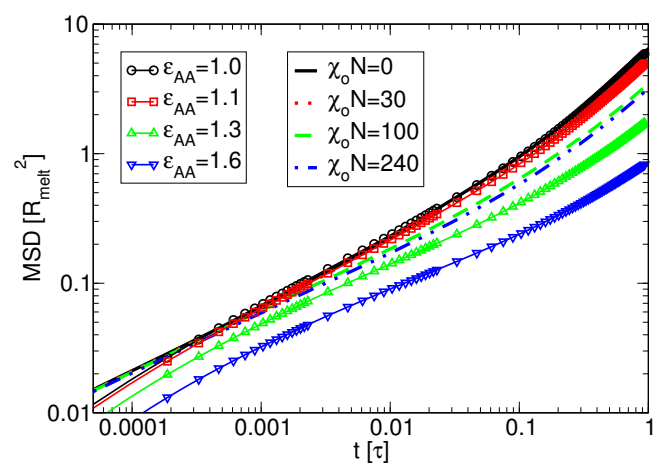

(a) MSD A-segments

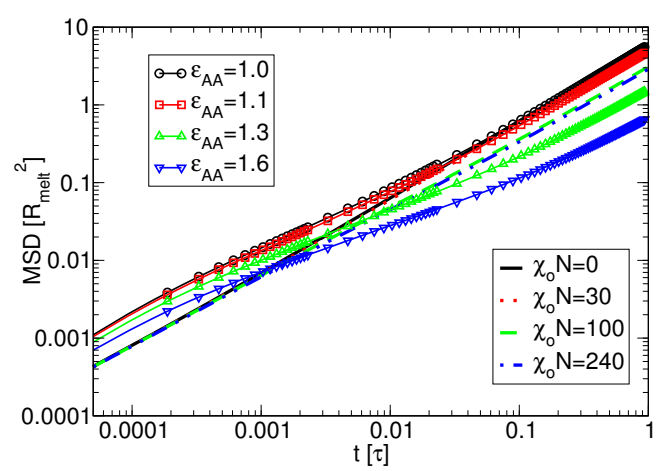

(c) MSD centers of mass

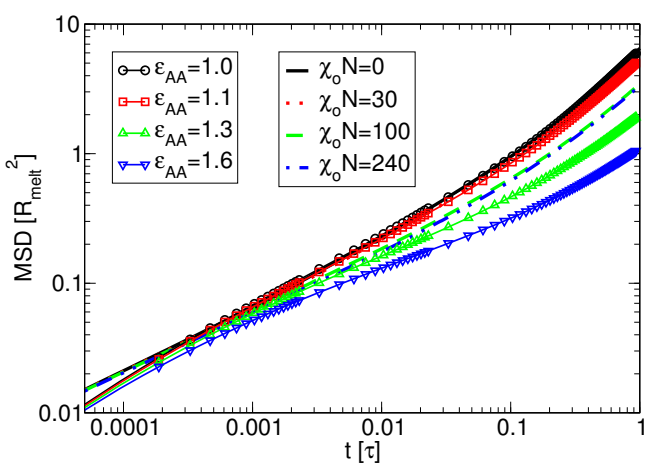

(b) MSD B-segments

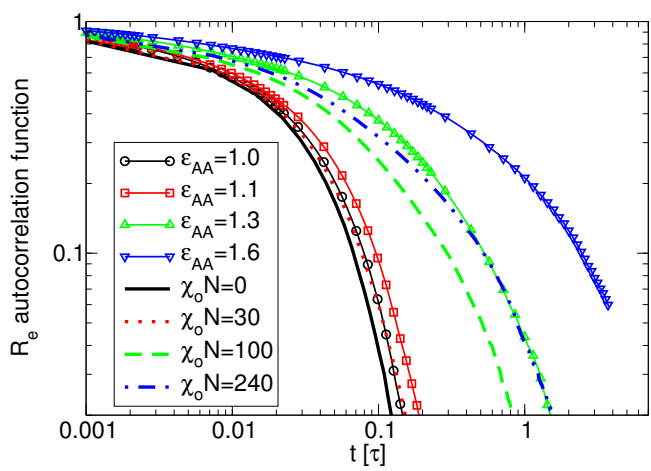

(d) $R_{e}$-acf

Figure 3.8: Dynamical properties, unscaled. Here we show some dynamical properties of our SCMF-systems, namely the MSD of 3.8(a) A-segments and 3.8(b) B-segments. In 3.8(d) the end-to-end vector autocorrelation function demonstrates the different time scales of these systems. The time scale is $\tau$ of the homopolymer systems. The different dynamics for short time scales of the two models are clearly visible.

\subsubsection{Equilibration Times After Quench}

We investigate the structure formation in response to a quench from the disordered phase in SCMF simulations in the NVT ensemble. For each set of parameters, we take an equilibrated homopolymer melt as the starting configuration, then distribute the polymer types, and let the system equilibrate, now with a different $\alpha$ and $\chi_{0} N$.

In figure 3.9, the time evolution of the pair correlation function is presented for three different values of $\chi_{0} N$ and, in the inset, the time evolution is presented for the 


\section{Bulk Properties}

structure factor. For the smallest interaction strength, $\chi_{0} N=30$, see figure 3.9(a), the pair correlation function and the structure factor show that the system equilibrates very fast; within $0.18 \tau$, its structure has reached an equilibrated state. When we look at the structure formation at the intermediate interaction strength, $\chi_{0} N=100$, we estimate from the pair correlation function, figure 3.9(b), that it takes $0.36 \tau$ to equilibrate the system. This equilibration time is compatible with the time evolution of the structure factor in figure 3.9(b), but it is more difficult to extract a time scale from $S(q)$. In case of the highest interaction strength, $\chi_{0} N=240$, it takes even longer to achieve an equilibrated structure. From the evolution of the pair correlation function, see figure 3.9(c), we estimate that the system takes about $0.73 \tau$ to equilibrate. The failure to reach equilibrium at $0.36 \tau_{\text {melt }}$ can, for this large incompatibility, also be inferred from the structure factor, cf. figure 3.9(c).

In the MD simulations, we adhered to the following simulation setup. We equilibrated a melt at $\varepsilon_{A A}=1.0$ and then quenched the system to the desired value of $\varepsilon_{A A}$. After the quench, we continued the simulation of the system in the NPT ensemble. We study the time evolution for the three different values of $\varepsilon_{A A}=1.1,1.3$ and 1.6, which we investigated in the last subsection. In figure 3.10, the time evolution of the pair correlation function is shown for these three different values of $\varepsilon_{A A}$ and the insets present the corresponding data for the structure factor. In qualitative agreement with the SCMF simulations, the time to form the equilibrium morphology increases with the incompatibility. At $\varepsilon_{A A}=1.1$, both measures of structure formation show an equilibration within $0.23 \tau$ see figure $3.10(\mathrm{a})$, For the intermediate incompatibility, $\varepsilon_{A A}=1.3$, the equilibration takes $0.7 \tau$, as depicted in figure $3.10(\mathrm{~b})$, and for the highest incompatibility, $\varepsilon_{A A}=1.6$, there is a further increase in the equilibration time to $1.7 \tau$.

Quantitatively, the SCMF model attains the equilibrium morphology faster than the Lennard-Jones bead-spring model when measured in units of the relaxation time of a single molecule in the disordered state. Moreover, the ratio of time scales

$$
\eta=\frac{\tau_{\mathrm{eq}, \mathrm{MD}} / \tau_{\mathrm{melt}, \mathrm{MD}}}{\tau_{\mathrm{eq}, \mathrm{SCMF}} / \tau_{\mathrm{melt}, \mathrm{SCMF}}}
$$

increases from $\eta=1.28$ at low incompatibilities to $\eta=2.33$ at high incompatibility. The relative slowing-down of the Lennard-Jones model is partially explained by the increase of the density in the segregated $A$-rich clusters. Upon further increase of $\varepsilon_{A A}$, the density inside the well-segregated $A$-domains increases and eventually leads 
to a vitrification. Note that the glass transition occurs in similar Lennard-Jones beadspring models around $1 / \varepsilon_{A A} \approx 0.42$ [BPBB99]. Since the slowing-down and the glass transition are related to the local, fluid-like packing, which is not captured by the soft, coarse-grained model, packing effects do not contribute to an increase of the equilibration time in the SCMF simulations.

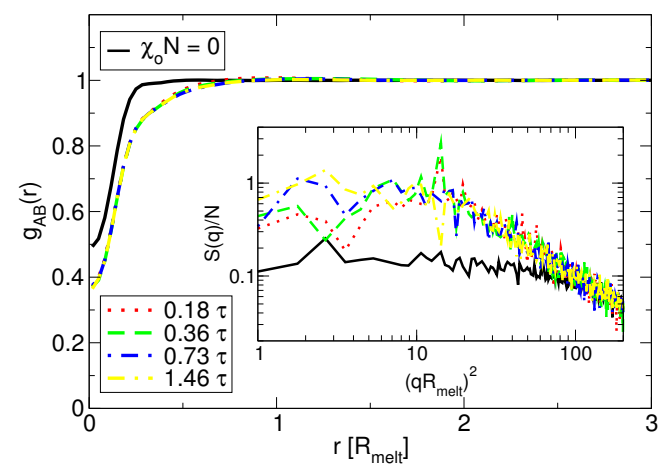

(a) $\chi_{0} N=30$

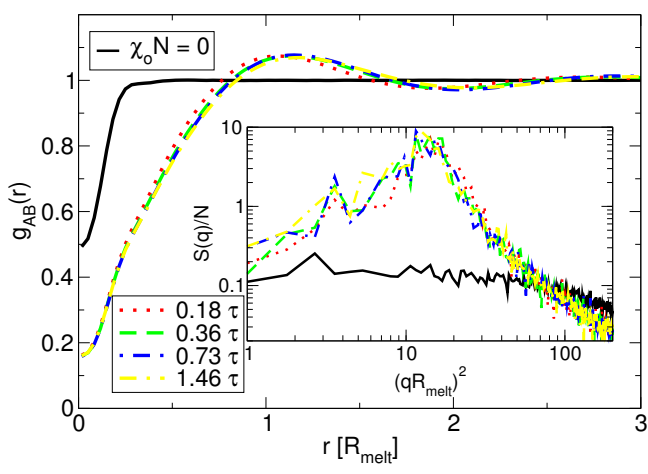

(b) $\chi_{0} N=100$

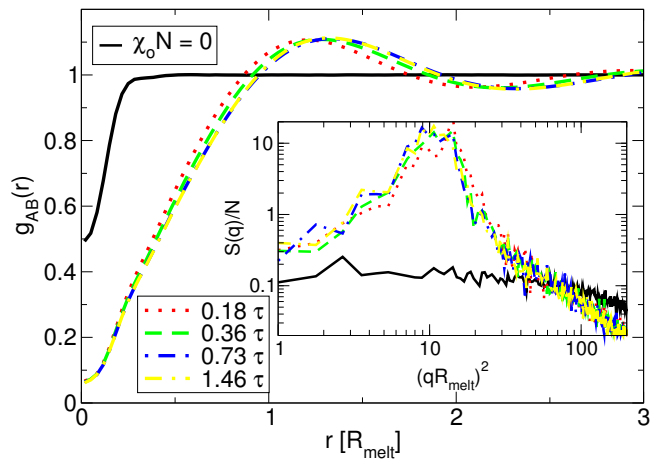

(c) $\chi_{0} N=240$

Figure 3.9: Time evolution of the radial A-B pair correlation function and the structure factor for three different sets of parameters in the SCMF model. $\kappa N$ was always set to 600, $\alpha$ and $\chi_{0} N$ are as follows: 3.9(a) $\alpha=0.01, \chi_{0} N=30$; 3.9(b) $\alpha=0.02, \chi_{0} N=100 ; 3.9$ (c) $\alpha=0.05, \chi_{0} N=240$. 


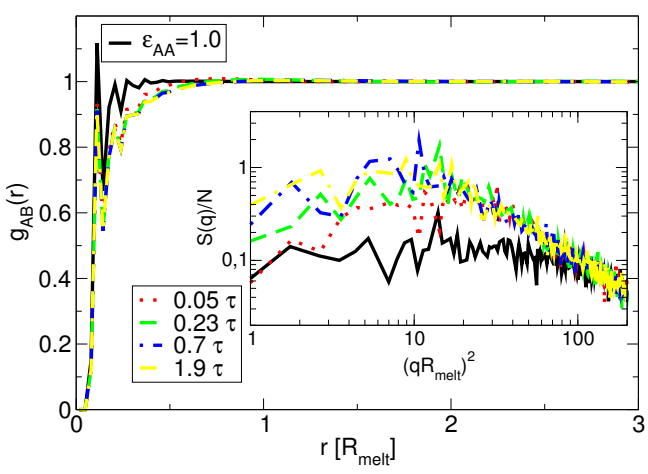

(a) $\varepsilon_{A A}=1.1$

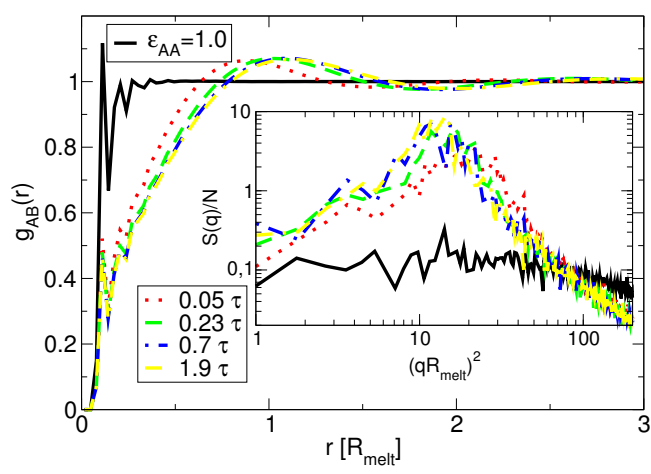

(b) $\varepsilon_{A A}=1.3$

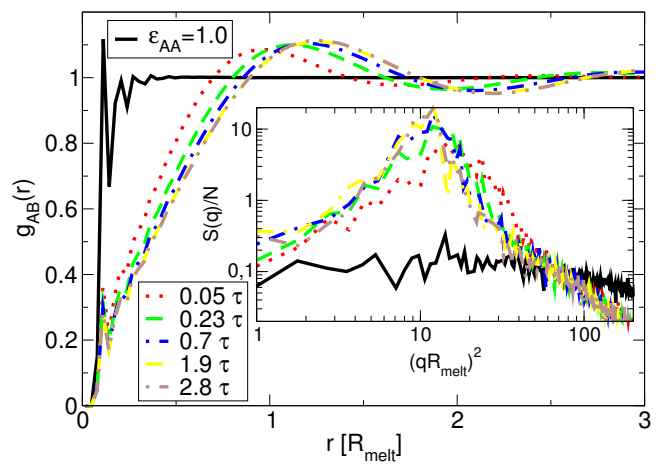

(c) $\varepsilon_{A A}=1.6$

Figure 3.10: Time evolution of the radial A-B pair correlation function and the structure factor for three different sets of parameters in the MD simulation. The parameter $\varepsilon_{A A}$ was set as follows: $3.10(\mathrm{a}) \varepsilon_{A A}=1.1 ; 3.10(\mathrm{~b}) \varepsilon_{A A} 1.3$; $3.10(\mathrm{c}) \varepsilon_{A A}=1.6$.

\subsubsection{Equilibration After Mapping SCMF on MD}

The previous subsections have shown that the Lennard-Jones bead-spring model and the soft, coarse-grained model agree in their equilibrium structure at intermediate and large length scales. Moreover, the SCMF simulations are computationally more efficient because the interactions are softer and the time scale is not affected by the liquidlike packing of segments. Therefore, it is tempting to use equilibrated configurations of the SCMF simulations for generating starting configurations for the Lennard-Jones bead-spring model. It is important to note that the configurations obtained by SingleChain-in-Mean-Field simulations capture intermolecular correlations on the scale of 


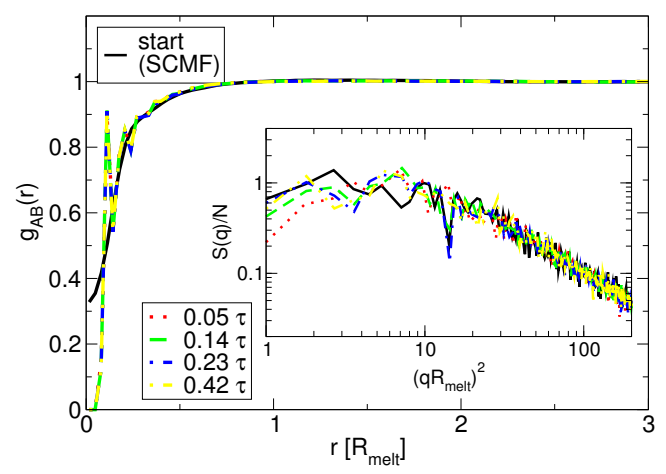

(a) $\varepsilon_{A A}=1.1$

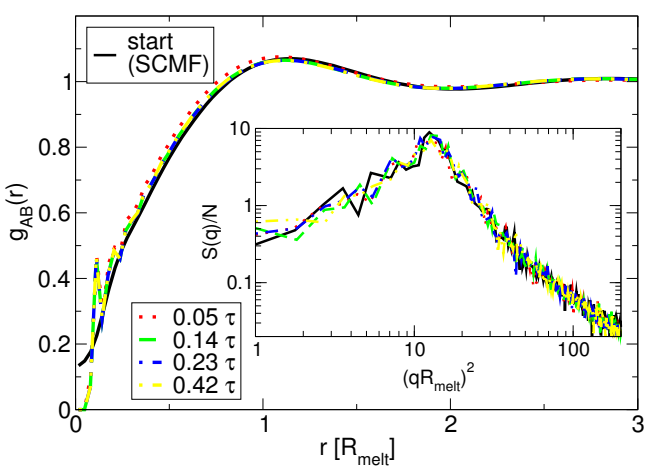

(b) $\varepsilon_{A A}=1.3$

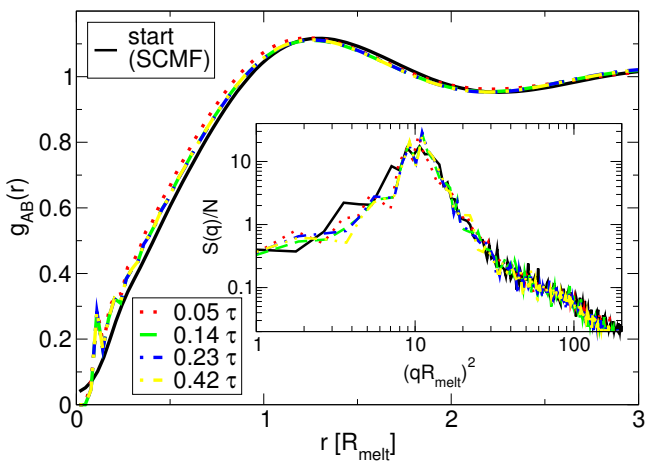

(c) $\varepsilon_{A A}=1.6$

Figure 3.11: Time evolution of $g_{A B}(r)$ and $S(q)$ in MD with SCMF starting configuration. The A-B pair correlation function and the structure factor are used to estimate the time needed to equilibrate the MD simulation with the equilibrated SCMF configuration used as an input for three different sets of parameters. In the SCMF model, $\kappa N$ was always set to $600, \alpha$ and $\chi_{0} N$ are as follows: 3.11(a) $\alpha=0.01, \chi_{0} N=30$; 3.11(b) $\alpha=0.02$, $\chi_{0} N=100 ; 3.11(\mathrm{c}) \alpha=0.05, \chi_{0} N=240$.

the molecule's extension, e.g., the correlation hole in the intermolecular pair correlation function. Otherwise a full single-chain relaxation time, $\tau$, would be required to establish those correlations [HM08]. All this gives us reason to expect a fast equilibration of the MD simulations when we start them with SCMF configurations.

Given an equilibrated soft, coarse-grained configuration with chain length $N=120$, we construct a starting configuration of the Lennard-Jones model with $N=60$ by representing the center of mass of two neighboring soft beads by a Lennard-Jones particle. 


\section{Bulk Properties}

Since the soft beads may overlap, we cannot simply switch on the harsh repulsion of the Lennard-Jones potential. First, we relax the system in the microcanonical ensemble, and restrict the maximum movement per bead and integration step to $0.05 \sigma$. At the same time, we rescale the velocities to put the temperature to $k_{B} T / \varepsilon_{B B}=1$ at the end of this simulation part by rescaling the velocities to the desired value. After 100 time steps, we switch on the thermostat and equilibrate the system for $t=0.05 \tau$. Finally, we simulate the system in the NPT-ensemble at $P=0$. The time evolution of the pair correlation function and the structure factor, cf. figure 3.11 , show that the structural equilibration of the system is very fast. Only the local fluid-like packing has to be established by the equilibration procedure. Both the morphology of $A$ and $B$ domains and the conformations on intermediate and large length scales as well as the correlation hole in the intermolecular pair correlation function are already captured by the SCMF simulations. For all three incompatibilities the desired structure is safely attained within three million integration steps which is equivalent to $0.14 \tau$. The results of the last two subsections can be summed up by figure 3.12. It depicts the position of the first peak of the $A B$ pair correlation function depending on the simulation time for all three equilibration schemes: SCMF with a random starting configuration, MD with a random starting configuration, and MD with SCMF starting configurations. The first two methods take between one and three $\tau$ to equilibrate, while the third scheme only needs a tenth of this equilibration time. To put this graph and the time scales into perspective, on a single CPU of the type Intel XEON X5570 at $2.93 \mathrm{GHz}, 1 \tau$ takes roughly 3600 hours of computing time when using LAMMPS with a system of this size, but only 36 hours when we use the SCMF method. Using SCMF configurations instead of random configurations for the equilibration in MD therefore reduces the needed computational effort by a factor of ten. 


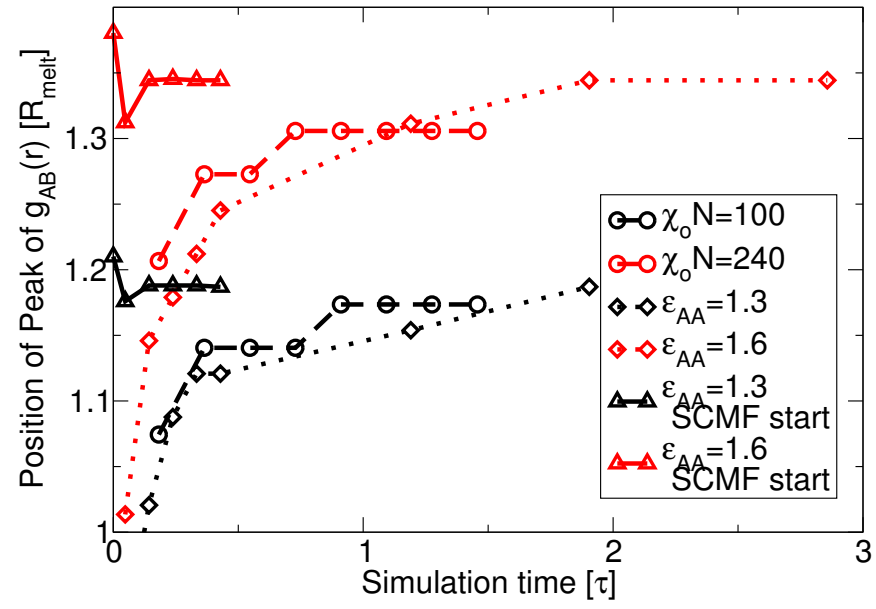

Figure 3.12: Comparison of equilibration. In this graph, the position of the first maximum of the A-B pair correlation function is used to gauge the time needed to equilibrate the SCMF system, the MD simulation with a random starting configuration and the MD simulation with the equilibrated SCMF configuration used as starting configuration. 


\section{Bulk Properties}

\subsection{Local Mechanical Properties}

Besides the local concentration and structural properties like the end-to-end vector and asymmetry of the polymer, we are also interested in the local mechanical properties of the random block copolymer melt. Be it shear modulus, bulk modulus, or Young's modulus, these quantities are among the most interesting for the design of new materials and also accessible to experiments. Furthermore, the heterogeneity of our system means that not only the mechanical properties of the whole simulation volume, but also these quantities at a more local level grant us insights into the properties of the random block copolymer melt. The measurement of local elastic properties has been done in the case of metallic glasses by using atomic force acoustic microscopy [ $\left.\mathrm{WBK}^{+} 11\right]$.

The method we use is from a paper of Yoshimoto et al., [Y $\left.\mathrm{YJV}^{+} 04\right]$, which itself is based on two papers by Lutsko, [Lut88] and [Lut89]. The formulation of local elastic constants is only valid at non-zero temperature and vanishing external strain. In [Lut89], there is also a derivation of the local elastic constants for zero temperature, but this is neither possible nor desirable in molecular dynamics simulations. The following approach can, in principle, also be applied to the soft, coarse-grained model, but the softness of the interaction and the concomitant lack of a glass transition temperature prevents solidification.

When a system is deformed linearly, each position $\vec{r}$ is transformed to $\vec{r}^{\prime}$ via

$$
\vec{r}^{\prime}=\mathbf{J} \vec{r}
$$

with the matrix $\mathbf{J}$, the strain tensor is

$$
\epsilon_{i j}=\frac{1}{2}\left(\mathbf{J}_{i l}^{T} \mathbf{J}_{l j}-\delta_{i j}\right) .
$$

To access the local mechanical properties, Lutsko starts with the local stress tensor, $\hat{\tau}(\vec{r})$, which can be derived from the momentum flux balance

$$
\frac{d}{d t} p_{i}(\vec{r})=\partial_{j} \hat{\tau}_{i j}(\vec{r})
$$

as in [Lut88]. In this section, Latin indices denote Cartesian components, that is $i, j \in$ $\{1,2,3\}$, while Greek indices are used to enumerate different particles. We utilize the Einstein convention for summation over equal Latin indices. The local momentum is defined by

$$
p_{i}(\vec{r})=\sum_{\alpha} p_{\alpha, i} \delta\left(\vec{r}-\vec{q}_{\alpha}\right)
$$


$\delta(x)$ is the Dirac delta function, $\vec{q}_{\alpha}$ is the position of particles $\alpha$ and $\vec{p}_{\alpha}$ is its momentum. The problem is easier to solve in momentum space, therefore we Fourier transform the equations (3.9) and (3.10) to

$$
\begin{aligned}
\frac{d}{d t} p_{i}(\vec{k}) & =k_{j} \cdot \hat{\tau}_{i j}(\vec{k}) \\
p_{i}(\vec{k}) & =\sum_{\alpha} p_{\alpha, i} \exp \left(i k_{l} q_{\alpha, l}\right) .
\end{aligned}
$$

We can now evaluate the time derivative in equation 3.11

$$
\frac{d}{d t} p_{i}(\vec{k})=\sum_{\alpha}\left(\dot{p}_{\alpha, i} \exp \left(i k_{l} q_{\alpha, l}\right)+i k_{j} \dot{q}_{\alpha, i} p_{\alpha, j} \exp \left(i k_{l} q_{\alpha, l}\right)\right)
$$

and transform the first part of the sum further by

$$
\begin{aligned}
\sum_{\alpha} \dot{p}_{\alpha, i} \exp \left(i k_{l} q_{\alpha, l}\right) & =-\sum_{\alpha} \frac{\partial U}{\partial q_{\alpha, i}} \exp \left(i k_{l} q_{\alpha, l}\right) \\
& =-i k_{j} \sum_{\alpha<\beta}\left(\frac{\partial U}{\partial q_{\alpha \beta}} q_{\alpha \beta}\right) \frac{q_{\alpha \beta, i} q_{\alpha \beta, j}}{q_{\alpha \beta}^{2}} \frac{\exp \left(i k_{l} q_{\alpha, l}\right)-\exp \left(i k_{l} q_{\beta, l}\right)}{i k_{m} q_{\alpha \beta, m}}
\end{aligned}
$$

with $\vec{q}_{\alpha \beta}$ being the vector connecting particles $\alpha$ and $\beta$, and $U$ the potential. We use these results to reshape equation (3.11) to

$$
\begin{aligned}
\hat{\tau}_{i j}(\vec{k})= & \sum_{\alpha} \frac{p_{\alpha, i} p_{\alpha, j}}{m} \exp \left(i k_{l} q_{\alpha, l}\right) \\
& -\sum_{\alpha<\beta}\left(\frac{\partial U}{\partial q_{\alpha \beta}} q_{\alpha \beta}\right) \frac{q_{\alpha \beta, i} q_{\alpha \beta, j}}{q_{\alpha \beta}^{2}} \frac{\exp \left(i k_{l} q_{\alpha, l}\right)-\exp \left(i k_{l} q_{\beta, l}\right)}{i k_{m} q_{\alpha \beta, m}} .
\end{aligned}
$$

We transform the stress tensor back to real space and receive

$$
\hat{\tau}_{i j}(\vec{r})=\sum_{\alpha} \frac{p_{\alpha, i} p_{\alpha, j}}{m} \delta\left(\vec{r}-\vec{q}_{\alpha}\right)+\sum_{\alpha<\beta}\left(\frac{\partial U}{\partial q_{\alpha \beta}}\right) \frac{q_{\alpha \beta, i} q_{\alpha \beta, j}}{q_{\alpha \beta}} g\left(\vec{q}_{\alpha}, \vec{q}_{\beta}, \vec{r}\right)
$$

with

$$
g\left(\vec{q}_{\alpha}, \vec{q}_{\beta}, \vec{r}\right)=\frac{1}{q_{\alpha \beta}} \delta\left(\left[\vec{r}-\vec{Q}_{\alpha \beta}\right]_{\perp}\right) \theta\left(\left[\vec{r}-\vec{q}_{\alpha}\right]_{\|}\right) \theta\left(-\left[\vec{r}-\vec{q}_{\beta}\right]_{\|}\right)
$$

enforcing that $\vec{r}$ has to be on the connecting line between $\vec{q}_{\alpha}$ and $\vec{q}_{\beta}$. In this equation $\vec{Q}_{\alpha \beta}$ is the center of mass of the two particles $\alpha$ and $\beta$, the index $\perp$ means that only the component of this vector perpendicular to $\vec{q}_{\alpha \beta}$ are taken into account and $\|$ results 


\section{Bulk Properties}

in the component parallel to $\vec{q}_{\alpha \beta}$ being used for this part of the calculation. $\theta(x)$ is the Heaviside step function.

We can now use the local stress tensor in equation 3.17 to calculate the local elastic modulus tensor. Calculating the canonical average before

$$
\tau_{i j}(\vec{r})=\left\langle\hat{\tau}_{i j}(\vec{r})\right\rangle_{\epsilon=0}
$$

and after

$$
\tau_{i j}^{\prime}\left(\vec{r}^{\prime}\right)=|\operatorname{det}(\mathbf{J})| \mathbf{J}_{i l}^{-1}\left\langle\hat{\tau}_{l m}\left(\vec{r}^{\prime}\right)\right\rangle_{\epsilon}\left(\mathbf{J}_{m j}^{T}\right)^{-1}
$$

the infinitesimal deformation, $\epsilon$ gives access to the local elastic constants

$$
C_{i j k l}(\vec{r})=\left.\lim _{\mathbf{J} \rightarrow \mathbb{1}_{3}} \frac{\partial \tau_{i j}\left(\vec{r}^{\prime}\right)}{\partial \epsilon_{k l}}\right|_{\vec{r}^{\prime}=\mathbf{J} \vec{r}}
$$

through the first derivative of local stress with respect to strain at vanishing strain. With

$$
\left(\mathbf{J}^{T}\right)_{k m} d \mathbf{J}_{m l}=d \epsilon_{k l}+d \mathbf{w}_{k l}
$$

where $\mathrm{w}$ is the rotational part and therefore vanishes, equation 3.21 therefore becomes

$$
C_{i j k l}(\vec{r})=\left.\frac{1}{2}\left(\frac{\partial \tau_{i j}(\mathbf{J} \vec{r})}{\partial \mathbf{J}_{k m}}\left(\mathbf{J}_{m l}^{T}\right)^{-1}+\frac{\partial \tau_{i j}(\mathbf{J} \vec{r})}{\partial \mathbf{J}_{m l}}\left(\mathbf{J}_{k m}^{T}\right)^{-1}\right)\right|_{\mathbf{J}=\mathbb{1}_{3}} .
$$

When evaluating equation 3.23 , it yields

$$
C_{i j k l}(\vec{r})=C_{i j k l}^{B}(\vec{r})-C_{i j k l}^{S}(\vec{r})+C_{i j k l}^{K}(\vec{r}) .
$$

The local elastic modulus tensor consists of three parts. The first one, called the Born term, is explicitly written as

$$
C_{i j k l}^{B}(\vec{r})=\sum_{\alpha<\beta}\left(\frac{\partial^{2} U}{\partial q_{\alpha \beta}^{2}}-\frac{1}{q_{\alpha \beta}} \frac{\partial U}{\partial q_{\alpha \beta}}\right) \frac{q_{\alpha \beta, i} q_{\alpha \beta, j} q_{\alpha \beta, k} q_{\alpha \beta, l}}{q_{\alpha \beta}^{2}} g\left(\vec{q}_{\alpha}, \vec{q}_{\beta}, \vec{r}\right) .
$$

This contains the higher derivatives of the potential and is the only term of the three which remains in the limit $T \rightarrow 0$. The second term, $C_{i j k l}^{S}(\vec{r})$, stems from the stress fluctuations

$$
C_{i j k l}^{S}(\vec{r})=\frac{V}{k_{B} T}\left[\left\langle\hat{\tau}_{i j}(\vec{r}) \hat{\tau}_{k l}\right\rangle-\left\langle\hat{\tau}_{i j}(\vec{r})\right\rangle\left\langle\hat{\tau}_{k l}\right\rangle\right]
$$

where $\hat{\tau}_{k l}$ is the global stress tensor. Simulations of poly(methyl methacrylate) [YPLdP05] showed the stress fluctuation term to be of comparable magnitude to the 
Born term for the shear modulus. Finally, the kinetic term, $C_{i j k l}^{K}(\vec{r})$, accounts for the contribution of the particles' kinetic energy

$$
C_{i j k l}^{K}(\vec{r})=2\langle\rho(\vec{r})\rangle k_{B} T\left(\delta_{i k} \delta_{j l}+\delta_{i l} \delta_{j k}\right)
$$

In the already mentioned simulations of PMMA [YPLdP05], the kinetic term was found to be two orders of magnitude smaller than the Born term.

Having successfully defined the local stress tensor, we adapt it to fulfill the requirements of a computer algorithm. Yoshimoto et al. [Y $\left.\mathrm{YJVW}^{+} 04\right]$ subdivided the simulation volume into equally sized cubic boxes to get a formula for the local elastic modulus tensor for each box. Since we also use non-cubic boxes later on, we reformulate the expressions into a more general form. The local stress tensor from equation 3.17 is averaged over a volume $V_{m}$ which is part of the total simulation volume, thus we obtain the locally averaged stress tensor $\hat{\tau}^{m}$

$$
\begin{aligned}
\hat{\tau}_{i j}^{m} & =\frac{1}{V_{m}} \int_{V_{m}} d^{3} r \hat{\tau}_{i j}(\vec{r}) \\
& =\frac{1}{V_{m}}\left[\sum_{\alpha \mid \vec{q}_{\alpha} \in V_{m}} \frac{p_{\alpha, i} p_{\alpha, j}}{m}+\sum_{\alpha<\beta}\left(\frac{\partial U}{\partial q_{\alpha \beta}}\right) \frac{q_{\alpha \beta, i} q_{\alpha \beta, j}}{q_{\alpha \beta}} \frac{l_{\alpha \beta}}{q_{\alpha \beta}}\right]
\end{aligned}
$$

where $l_{\alpha \beta}$ is the length of the segment of the line connecting particle $\alpha$ and $\beta$ which is inside the volume $V_{m}$. If the connecting line does not pass through the volume, this is zero, this ensures that only particles inside the volume or for which the connecting line passes through $V_{m}$ are used for this averaging. When using the whole simulation volume as $V_{m}$, we recover the total stress tensor of the system. The local elastic modulus tensor of equation 3.24 is likewise averaged over the volume $V_{m}$ to obtain $C_{i j k l}^{m}$

$$
C_{i j k l}^{m}=C_{i j k l}^{B, m}-C_{i j k l}^{S, m}+C_{i j k l}^{K, m}
$$

The Born term, averaged over $V_{m}$, is

$$
\begin{aligned}
C_{i j k l}^{B, m} & =\frac{1}{V_{m}} \int_{V_{m}} d^{3} r C_{i j k l}^{B}(\vec{r}) \\
& =\frac{1}{V_{m}} \sum_{\alpha<\beta}\left(\frac{\partial^{2} U}{\partial q_{\alpha \beta}^{2}}-\frac{1}{q_{\alpha \beta}} \frac{\partial U q_{\alpha \beta}}{\partial q_{\alpha \beta}}\right) \ldots \\
& \ldots \cdot \frac{q_{\alpha \beta, i} q_{\alpha \beta, j} q_{\alpha \beta, k} q_{\alpha \beta, l}}{q_{\alpha \beta}^{2}} \frac{l_{\alpha \beta}}{q_{\alpha \beta}},
\end{aligned}
$$




\section{Bulk Properties}

the stress fluctuation term becomes

$$
C_{i j k l}^{S, m}=\frac{V}{k_{B} T}\left[\left\langle\hat{\tau}_{i j}^{m} \hat{\tau}_{k l}\right\rangle-\left\langle\hat{\tau}_{i j}^{m}\right\rangle\left\langle\hat{\tau}_{k l}\right\rangle\right]
$$

and the kinetic term

$$
C_{i j k l}^{K, m}=2\left\langle\rho^{m}\right\rangle k_{B} T\left(\delta_{i k} \delta_{j l}+\delta_{i l} \delta_{j k}\right),
$$

where $\rho^{m}$ is the number density of particles in $V_{m}$. We have not specified the shape and size of $V_{m}$ further. This is done with a specific system in mind, since it should neither contain too few particles, nor should it be so big as to just mirror the properties of the whole system. We use boxes which contain on average 31.2 particles. The size of a box depends on the value of $\varepsilon_{A A}$ and the temperature, it is on the order of $0.27 R_{\text {melt }}^{3}$. The bulk properties are calculated from this method by integrating over the whole simulation box.

\subsubsection{Voigt Notation and Moduli}

To reduce the number of indices in the equations, we resort to the Voigt notation. In this notation, a pair of indices is replaced by just one number. In this way, the secondorder stress and strain tensor become, due to their symmetry, 6-dimensional vectors and the fourth-order tensor $C_{i j k l}$ turns into a second-order tensor. The replacement is chosen as in [Ray88]:

$$
11 \rightarrow 1 ; 22 \rightarrow 2 ; 33 \rightarrow 3 ; 23 \rightarrow 4 ; 13 \rightarrow 5 ; 12 \rightarrow 6 .
$$

The two moduli we are most interested in are the shear modulus and the bulk modulus. Using the abbreviations

$$
\bar{C}_{11}=\frac{1}{3}\left(C_{11}+C_{22}+C_{33}\right)
$$

and

$$
\bar{C}_{44}=\frac{1}{3}\left(C_{44}+C_{55}+C_{66}\right),
$$

we can write down the averaged shear modulus as

$$
G=\bar{C}_{44}
$$

and the bulk modulus[Ray88]

$$
K=\bar{C}_{11}-\frac{4}{3} \bar{C}_{44}
$$




\subsubsection{Bulk Modulus}

The bulk modulus, also called the incompressibility, can also be determined from thermodynamic variables, not only from the microscopic properties. The isothermal compressibility is defined as

$$
\kappa_{T}=-\frac{1}{V}\left(\frac{\partial V}{\partial P}\right)_{T}
$$

and the bulk modulus $K$ is its inverse

$$
K=\frac{1}{\kappa_{T}}
$$

The partition function of the NPT-ensemble is

$$
Z_{N P T}=\int_{0}^{\infty} d V \exp (-\beta P V) Z_{N V T}
$$

with the canonical partition function $Z_{N V T}$ and $\beta=\frac{1}{k_{B} T}$. The expectation value of the volume in the NPT-ensemble is calculated as usual from

$$
\langle V\rangle=-\frac{1}{\beta} \frac{\partial \ln Z_{N P T}}{\partial P} .
$$

The second logarithmic derivative of the NPT partition function then yields

$$
\frac{\partial^{2} \ln Z_{N P T}}{\partial P^{2}}=\frac{1}{Z_{N P T}} \frac{\partial^{2} Z_{N P T}}{\partial P^{2}}-\frac{1}{Z_{N P T}}\left(\frac{\partial Z_{N P T}}{\partial P}\right)^{2}=\beta^{2}\left\langle V^{2}\right\rangle-\beta^{2}\langle V\rangle^{2}
$$

Thus, we can calculate the isothermal compressibility of the whole system from the volume fluctuations.

$$
\kappa_{T}=-\frac{1}{\langle V\rangle}\left(\frac{\partial\langle V\rangle}{\partial P}\right)_{T}=\beta \frac{\left\langle V^{2}\right\rangle-\langle V\rangle^{2}}{\langle V\rangle}
$$

which are a part of the MD simulation scheme we employ.

This measurement of $\kappa_{T}$ also explains the choice of $\kappa N=600$. From the simulations in the first section of this chapter, we calculate $K=\frac{1}{\kappa_{T}}=10.2$ for a homopolymer melt at $k_{B} T=1.0$. This, together with the relation in equation (2.17), gives us a value for $\kappa N$ of 750 . These values are very high for SCMF simulations, which normally employ lower values on the order of $\kappa N=50$ [DM06]. 


\section{Bulk Properties}

\subsubsection{Shear Modulus}

The approach to compare the shear modulus obtained from the local elastic modulus tensor with a different method is not as straightforward as for the bulk modulus. We use the local stress tensor from equation (3.28) for this purpose. The shear modulus is defined as the derivative of shear stress with respect to shear strain

$$
C_{44}=\frac{\partial \tau_{4}}{\partial \epsilon_{4}} .
$$

We discussed this using equilibrium molecular dynamics, but we can also apply nonequilibrium molecular dynamics [Y $\left.\mathrm{YJV}^{+} 04\right]$. To this end, we shear the system and measure the change in shear stress

$$
\Delta \tau_{4}\left(\epsilon_{4}\right)=\tau_{4}(0)-\tau_{4}\left(\epsilon_{4}\right)
$$

For small shear strain, where the stress depends linearly on the strain, we can simply take the ansatz

$$
\Delta \tau_{4}\left(\epsilon_{4}\right)=C_{44} \epsilon_{4}+c_{0}
$$

with a constant $c_{0}$ and fit this equation to the data. This is an independent approach for determining the shear modulus, which we also use to try to find a correlation between the local mechanical stress tensor and the response to external strain.

\subsection{Global Moduli}

\subsubsection{Glass Transition}

The glass transition temperature for a Lennard-Jones bead-spring model was found to be between $k_{B} T=0.425$ for $P=0.5$ and $k_{B} T=0.49$ for $P=2.0$ [BPBB99]. We quench our system to access the local mechanical properties in the following section. Thus, we look for the indication of a glass transition. In many different systems, granular matter [NCH97], colloids [ $\mathrm{ZSVB}^{+}$06], Lennard-Jones liquids [ $\mathrm{DGP}^{+}$99], and polymers [Roe94, BPBBD98], the emergence of a plateau in the mean-squared displacement of polymers has been identified with being close to the glass transition temperature. If the plateau is finite,the system is above the glass transition. In our simulations, we look as an example at the homopolymer melt.

In figure 3.13, the mean-squared displacement is shown for three different temperatures. For the temperatures we investigate later on, $k_{B} T=0.3$ and $k_{B} T=0.1$, there is 
a clearly discernible plateau over several orders of magnitude in time. For $k_{B} T=0.3$, the plateau ends after $\sim 0.02 \tau$. If we want to measure the elastic properties of the system, its stress must decorrelate on a time scale, which is much larger than the time it takes us to measure these properties. Thus, the extremely slowed-down dynamics of the melt at these temperatures is sufficient for our purposes.

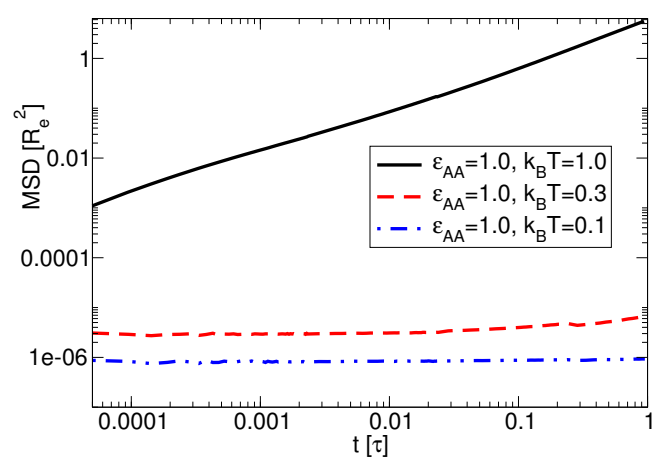

Figure 3.13: The mean-squared displacement for $k_{B} T=0.1$ and $k_{B} T=0.3$ in the homopolymer melt with $k_{B} T=1.0$ as the reference point. The dynamics of the melt are slowed down so that we are able to measure local mechanical properties.

\subsubsection{Shear and Bulk Modulus}

We start with the examination of the melt's bulk properties. As noted in subsection 3.2.1, we investigate the bulk and the shear modulus of the system. In order to measure the quantities, we take equilibrated configurations from section 3.1. The system is then quenched over a simulation run of $10^{6}$ simulation steps below the glass transition temperature, either to a value of $k_{B} T=0.1$ or $k_{B} T=0.3$. The system continues to run in the NPT ensemble at vanishing pressure for $4 \cdot 10^{6}$ steps. We take the NPT ensemble at this point so that the system can contract and avoid fissures or holes in the structure. After this, we let it run for $10^{6}$ steps and write out the volume every 500 time steps $-2.4 \cdot 10^{-5} \tau-$ to calculate the bulk modulus, see table 3.3 . We continue with the equilibration run after switching to the canonical ensemble for $6 \cdot 10^{6}$ time steps. Then we continue with taking data for the calculation of the elastic modulus tensor. The configurations are taken every 200 time steps $-9.5 \cdot 10^{-6} \tau-$, over $6 \cdot 10^{6}$ time steps, thus we average over $3 \cdot 10^{4}$ configurations in total. For computational 


\section{Bulk Properties}

reasons, we subdivide the data run into 24 parts. In each of these parts, we calculate the elastic modulus tensor according to equations (3.29), (3.30), 3.31), (3.32). The end result represents an average over these configurations. The bulk modulus in table 3.3 is calculated as described in equation (3.37) and the shear modulus in table 3.4 is calculated as in equation 3.36. We have to note that the ensemble average of the bulk modulus taken in the isobaric-isothermal ensemble and the one taken in the canonical ensemble can differ since we are not working in the thermodynamic limit. To determine the shear modulus via NEMD, see subsection 3.2 .3 , we apply a constant shear deformation in the $y-z$ plane. The shear strain is increased by $\Delta \epsilon_{4}=5 \cdot 10^{-4}$ every $10^{4}$ time steps. Again, the stress is calculated as an average over this interval with a step size of 200 time steps. The linear regime holds at least up to a shear strain of $\epsilon_{4}=10^{-2}$, and we use these points to calculate the shear modulus of the system as described in subsection 3.2.3.

The values of the bulk modulus calculated in table 3.3 in the two different ensembles differ by at most seven percent, for the parameters $k_{B} T=0.1$ and $\varepsilon_{A A}=1.0$. If we were only interested in the bulk modulus of the whole system, the method using the volume fluctuations would be the computationally preferable approach, as we can see from the description of the method used.

When looking at the results for the shear modulus, the results of the two methods agree even better, see table 3.4 . Again, the computational effort for the more specialized approach, this time the NEMD simulation, is at least an order of magnitude smaller than for the more general approach of calculating the whole elastic modulus tensor. When differentiating between the contributions of the bonded and the nonbonded interactions to the Born term of the shear modulus, see equation (3.30), for a homopolymer melt, the results show that the non-bonded interactions are far more important. At $k_{B} T=0.1$, the Born term has a value of 68.4 , with the bonded interaction contributing 2.9 and the non-bonded ones 65.5 to the final result. At $k_{B} T=0.3$, we find that the bonded interactions' contribution to the Born term is not as dependent on the temperature. The total value of 64.9 consists of 2.9 from the bonded interactions and 62.0 from the non-bonded interactions.

Now we put these values in relation with experimental measurements of the bulk modulus. For glassy Polystyrene, Meng et al. [ $\left.\mathrm{MBO}^{+} 09\right]$ measures values of the bulk modulus of $3 \mathrm{GPa}$ and higher in a glassy system of $T=333 \mathrm{~K}$ and of $1 \mathrm{GPa}$ and higher in a melt state. Their Polystyrene melt has the parameters $M_{n}=92.8 \mathrm{~kg} / \mathrm{mol}$ 
and $M_{W}=92.8 \mathrm{~kg} / \mathrm{mol}$ and they measure a glass transition temperature of $T_{g}=$ $368 \mathrm{~K}$ at room pressure. With $T_{g}=0.4 \varepsilon_{A A}$ in our simulations, and a Kuhn length, equal to our coarse-grained monomer size of $\sigma=5 \mathrm{~nm}$, [DS04], we can transform our results into MKSA units. With the Boltzmann constant $k_{B}=1.38 \cdot 10^{-23}$, one of our Lennard-Jones units for the moduli is

$$
\frac{k_{B} T_{g}}{0.4} \frac{1}{\sigma^{3}}=0.1 M P a
$$

With the calculated bulk modulus, this would result in a value of 5 to $6 \mathrm{MPa}$, three orders of magnitude smaller than the experimental values. We see that even the LennardJones bead-spring model is far too soft when calculating the bulk modulus. The glass having a bulk modulus which is higher by a factor of 6 , compared to the molten state, is commensurate with the experimental observations since we measure at a temperature which is smaller relative to the glass transition temperature.

Looking at different results for the shear modulus of polystyrene [YW71], gives us a value of the shear modulus at $T=200 \mathrm{~K}$ of $1.5 \mathrm{GPa}$. This experiment was performed at vanishing pressure, compared to the ones applied in $\left[\mathrm{MBO}^{+} 09\right]$, we can still assume the shear modulus to be lower by a factor of two. For our simulations, however, we typically find the shear modulus to be lower by a factor of four. Thus, in our simulations, we miss a factor of two between shear and bulk modulus, when compared to experimental values.

As a summary of this calculation, we can state that the Lennard-Jones bead-spring model is too soft when we consider it on the length scale we assume for the purpose of coarse-graining. 


\section{Bulk Properties}

\begin{tabular}{|l|l|l|l|l|}
\hline & \multicolumn{2}{|c|}{$k_{B} T=0.3$} & \multicolumn{2}{c|}{$k_{B} T=0.1$} \\
\hline$\varepsilon_{A A}$ & NPT & NVT & NPT & NVT \\
\hline 1.0 & $47.9 \pm 3.2$ & $50.4 \pm 3.5$ & $65.6 \pm 2.2$ & $65.3 \pm 3.6$ \\
\hline 1.1 & $50.0 \pm 4.7$ & $52.2 \pm 2.6$ & $67.0 \pm 1.3$ & $67.2 \pm 3.8$ \\
\hline 1.3 & $57.8 \pm 1.8$ & $58.3 \pm 6.4$ & $72.5 \pm 1.5$ & $72.9 \pm 3.2$ \\
\hline 1.6 & $62.2 \pm 0.5$ & $64.2 \pm 3.4$ & $79.1 \pm 1.3$ & $78.4 \pm 3.4$ \\
\hline
\end{tabular}

Table 3.3: Bulk modulus as calculated from the local elastic modulus tensor and the volume fluctuations for $k_{B} T=0.1$ and $k_{B} T=0.3$ at different interaction strengths $\varepsilon_{A A}$. Although different ensembles are used for the calculation of these moduli, they differ only by a few percentage points.

\begin{tabular}{|l|l|l|l|l|}
\hline & \multicolumn{2}{|c|}{$k_{B} T=0.3$} & \multicolumn{2}{c|}{$k_{B} T=0.1$} \\
\hline$\varepsilon_{A A}$ & NEMD & Tensor & NEMD & Tensor \\
\hline 1.0 & $11.9 \pm 0.7$ & $11.5 \pm 1.1$ & $14.6 \pm 0.9$ & $14.3 \pm 1.4$ \\
\hline 1.1 & $12.3 \pm 0.7$ & $12.6 \pm 0.8$ & $15.2 \pm 0.3$ & $15.7 \pm 1.0$ \\
\hline 1.3 & $14.3 \pm 0.7$ & $15.0 \pm 2.0$ & $16.5 \pm 1.2$ & $17.2 \pm 1.3$ \\
\hline 1.6 & $16.0 \pm 0.5$ & $15.9 \pm 1.0$ & $18.4 \pm 0.5$ & $18.9 \pm 0.9$ \\
\hline
\end{tabular}

Table 3.4: Shear modulus as calculated from the local elastic modulus tensor and NEMD simulations at a temperature of $k_{B} T=0.3$ and $k_{B} T=0.1$ at different interaction strength $\varepsilon_{A A}$. The values calculated by these two different methods agree very well. 


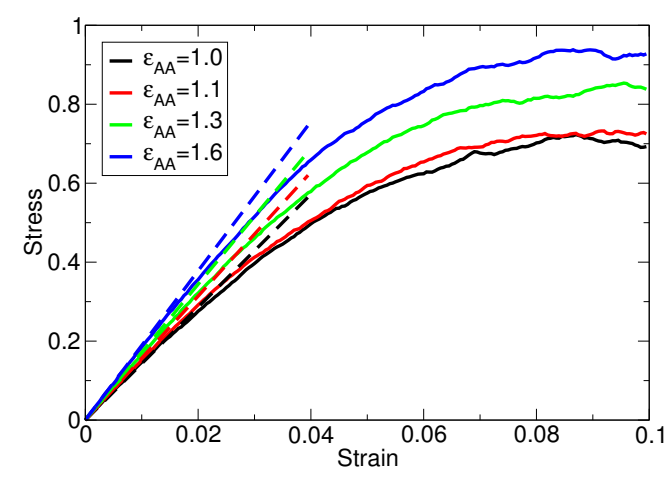

(a) $k_{B} T=0.1$

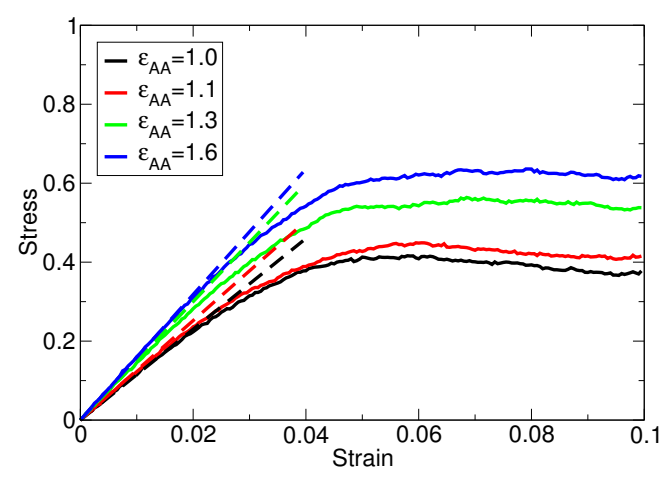

(b) $k_{B} T=0.3$

Figure 3.14: The shear stress for two different temperatures, $k_{B} T=0.1$ and $k_{B} T=$ 0.3. The dashed lines represent the expected result for the shear stress in the linear regime from the calculations of the elastic modulus tensor. The shear stress measured while actually shearing the system is given by the solid line. As we can see from these figures, assuming linear behaviour of the shear stress up to a shear strain of $\epsilon_{4}=0.01$ is reasonable.

\subsection{Local Moduli}

In this section we take the local structure into account to determine its influence on the local moduli. First we look at the different mechanical properties depending on the local concentration of the two monomer species. Later on, we investigate a correlation between the local shear modulus and the shear stress.

\subsubsection{Shear and Bulk Modulus}

As the structure of the random block copolymer melt becomes microemulsion-like for higher incompatibilities of the monomers, domains of high concentration of $A$ and $B$ monomers form. We are interested in the difference of the mechanical properties of these domains and the interfaces which separate them. To this end, we subdivide our simulation volume of $\approx\left(6.25 R_{\text {melt }}\right)^{3}$ into $20^{3}$ boxes and assign each box to one of these three groups. For a box to be $A$-rich, it has to contain at least ninety percent $A$-monomers, a $B$-rich box contains at least ninety percent $B$-monomers. The rest of these boxes define the interfacial regions. The procedure follows the same course as in 


\section{Bulk Properties}

section 3.3 for the elastic modulus tensor and for the NEMD simulations. This time, however, we average not over the whole simulation volume, but only over the three types of boxes we have just defined. One box contains on average 31.2 monomers, which is 0.52 chains. We include $\varepsilon_{A A}=1.1$ in our analysis, a disordered system. Therefore, the difference between the regions is smaller than for $\varepsilon_{A A}=1.3$ and $\varepsilon_{A A}=$ 1.6. A more detailed analysis of the number of monomers per box and the number of boxes assigned to each of the three categories is given in table 3.5. For $\varepsilon_{A A}=1.1$, only ten percent of the boxes are found to be $A$ - or $B$-rich. For $\varepsilon_{A A}=1.3$, this value approaches fifty percent, and the even stronger segregation for $\varepsilon_{A A}=1.6$ becomes clearly visible with more than sixty percent of boxes being considered either $A$ - or $B$-rich.

The bulk modulus, see table 3.6 and figure 3.15 , in $A$-rich regions rises with growing $\varepsilon_{A A}$, as we expect it to. The bulk modulus in the interfacial region and the $B$-rich region exhibits a similar behaviour. At the same time, the quenched systems, $k_{B} T=0.3$ and $k_{B} T=0.1$, do not become denser with rising $\varepsilon_{A A}$. The volume of the simulation box in the NPT ensemble is $\left(6.25 R_{\text {melt }}\right)^{3}$ for $\varepsilon_{A A}=1.0$ and $k_{B} T=0.3$, the same as for $\varepsilon_{A A}=1.6$ at this temperature.

The shear modulus, see table 3.7 and figure 3.16 , in the $A$-rich region and the interfacial region also grows with rising $\varepsilon_{A A}$. However, in the $B$-rich region, it does not exhibit a change as big as the bulk modulus. Considering the accuracy of our calculations, we take the shear modulus in the $B$-rich regions as being independent of $\varepsilon_{A A}$. While the high value of $\varepsilon_{A A}$ leads to a system which is as a whole more rigid with respect to compression, the shear modulus grows only in the regions where this stronger interaction takes effect.

We have not only applied the calculation of the elastic modulus tensor to this problem, but have also used NEMD simulations to determine this quantity. We proceed as in section 3.3, where we shear the system with a constant sequence of step strains. This time, we calculate the local stress tensor for each box, correlating the local stress with the global strain. We average over boxes of the same type, $A$-rich, $B$-rich, and interface, to receive the stress-strain curves. These curves, in comparison with the shear modulus according to the local elastic modulus tensor, see figure 3.17, are used to calculate the shear modulus in the NEMD scheme for each region. The values are given for comparison in tables 3.7. Now in contrast to the global shear modulus, where the maximum difference is seven percent, see table 3.4, this difference rises to ten percent. 


\begin{tabular}{|l|l|l|l|l|l|l|}
\hline & \multicolumn{2}{|c|}{$A$-rich } & \multicolumn{2}{c|}{ Interface } & \multicolumn{2}{c|}{$B$-rich } \\
\hline$\varepsilon_{A A}$ & $\begin{array}{l}\text { number } \\
\text { of boxes }\end{array}$ & $\begin{array}{l}\text { monomers } \\
\text { per box }\end{array}$ & $\begin{array}{l}\text { number } \\
\text { of boxes }\end{array}$ & $\begin{array}{l}\text { monomers } \\
\text { per box }\end{array}$ & $\begin{array}{l}\text { number } \\
\text { of boxes }\end{array}$ & $\begin{array}{l}\text { monomers } \\
\text { per box }\end{array}$ \\
\hline & \multicolumn{6}{|c|}{$k_{B} T=0.3$} \\
\hline 1.1 & 406 & 31.1 & 7170 & 31.2 & 424 & 31.1 \\
\hline 1.3 & 2075 & 31.3 & 3824 & 31.2 & 2101 & 31.1 \\
\hline 1.6 & 2452 & 31.4 & 3129 & 31.2 & 2419 & 31.1 \\
\hline & \multicolumn{6}{|c|}{$k_{B} T=0.1$} \\
\hline 1.1 & 398 & 31.0 & 7193 & 31.2 & 409 & 31.0 \\
\hline 1.3 & 1974 & 31.3 & 4077 & 31.2 & 1949 & 31.2 \\
\hline 1.6 & 2438 & 31.2 & 3142 & 31.2 & 2420 & 31.2 \\
\hline
\end{tabular}

Table 3.5: The number of boxes defined as $A$-rich, interface, and $B$-rich and the average number of monomers contained therein. The results are averaged over the data acquisition steps for the calculation of the elastic modulus tensor.

\begin{tabular}{|l|l|l|l|l|l|l|}
\hline & \multicolumn{3}{|c|}{$k_{B} T=0.3$} & \multicolumn{3}{c|}{$k_{B} T=0.1$} \\
\hline$\varepsilon_{A A}$ & $A$-rich & Interface & $B$-rich & $A$-rich & Interface & $B$-rich \\
\hline 1.1 & $53.9 \pm 7.7$ & $52.1 \pm 2.8$ & $51.3 \pm 8.6$ & $70.9 \pm 14.9$ & $67.1 \pm 3.6$ & $64.4 \pm 13.1$ \\
\hline 1.3 & $61.7 \pm 10.3$ & $58.6 \pm 8.0$ & $54.6 \pm 8.4$ & $75.7 \pm 6.5$ & $72.1 \pm 3.8$ & $71.3 \pm 5.7$ \\
\hline 1.6 & $70.5 \pm 5.6$ & $63.6 \pm 4.2$ & $58.5 \pm 4.4$ & $82.0 \pm 6.3$ & $80.0 \pm 5.4$ & $72.3 \pm 4.9$ \\
\hline
\end{tabular}

Table 3.6: The bulk modulus for $A$-rich regions, the interfacial regions, and $B$-rich regions as calculated from the local elastic modulus tensor. An $A$-rich box in the simulation volume contains at least ninety percent $A$-monomers, a $B$-rich box contains at least ninety percent $B$-monomers and the interface boxes fulfill neither condition. 


\section{Bulk Properties}

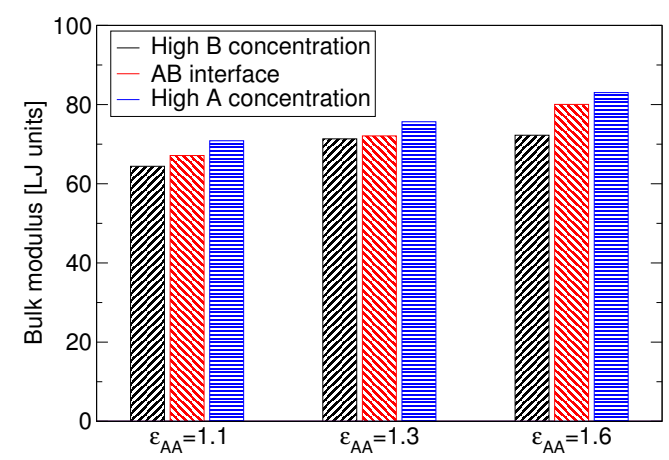

(a) $k_{B} T=0.1$

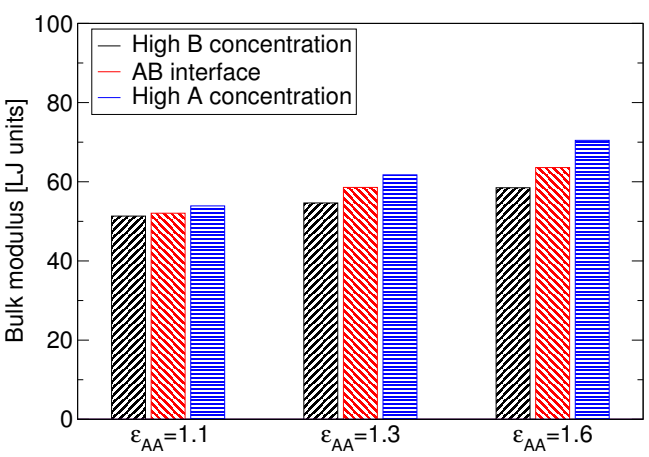

(b) $k_{B} T=0.3$

Figure 3.15: The bulk modulus for two different temperatures, $k_{B} T=0.1$ and $k_{B} T=$ 0.3. For each temperature, the bulk modulus was calculated in the three regions of high $\mathrm{A}$ concentration, high $\mathrm{B}$ concentration, and the $\mathrm{AB}$ interface via the elastic modulus tensor, see equation (3.37).

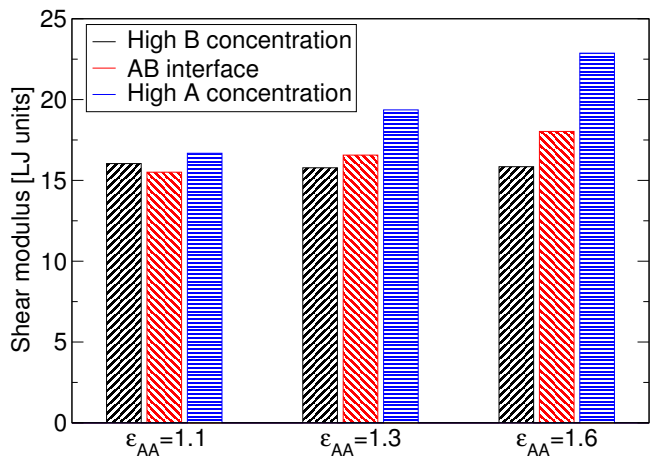

(a) $k_{B} T=0.1$

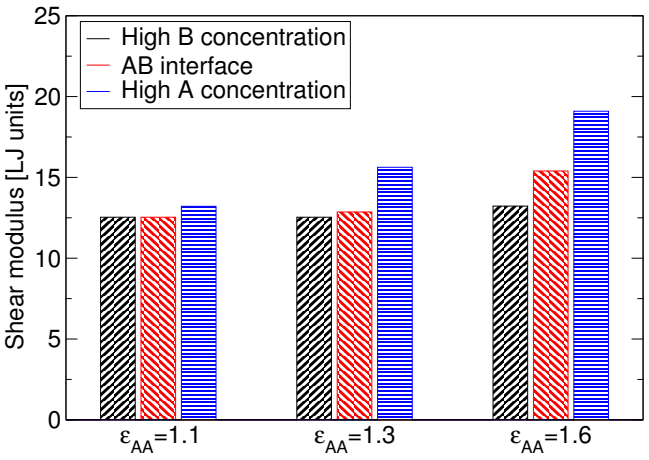

(b) $k_{B} T=0.3$

Figure 3.16: The shear modulus for two different temperatures, $k_{B} T=0.1$ and $k_{B} T=0.3$. For each temperature, the shear modulus was calculated in the three regions of high A concentration, high B concentration, and the $\mathrm{AB}$ interface via the elastic modulus tensor. 


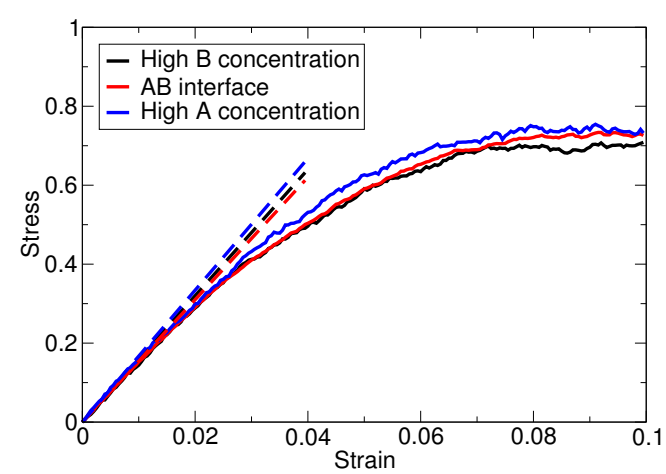

(a) $\varepsilon_{A A}=1.1$

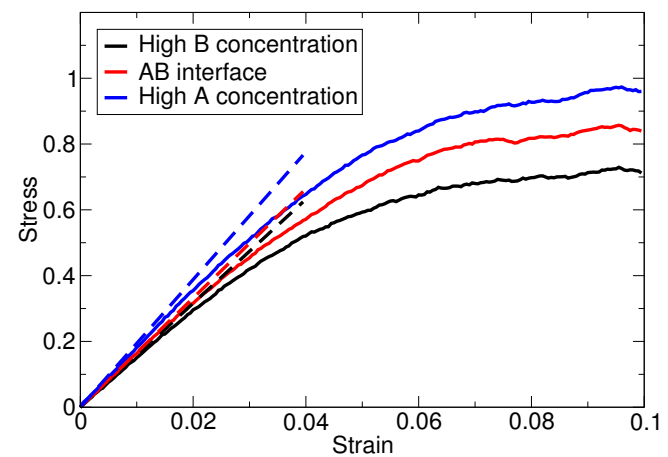

(c) $\varepsilon_{A A}=1.3$

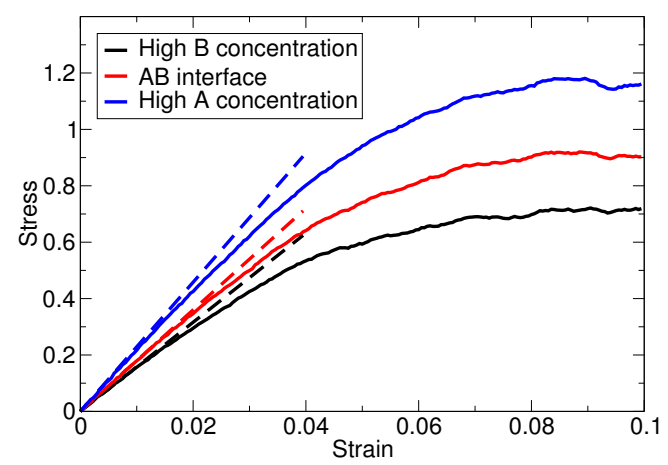

(e) $\varepsilon_{A A}=1.6$

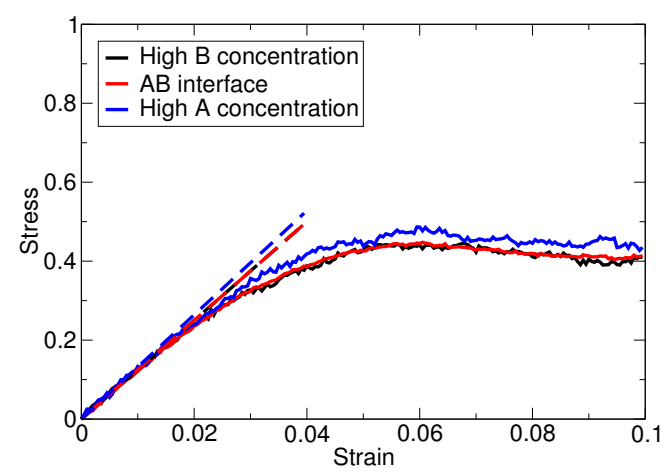

(b) $\varepsilon_{A A}=1.1$

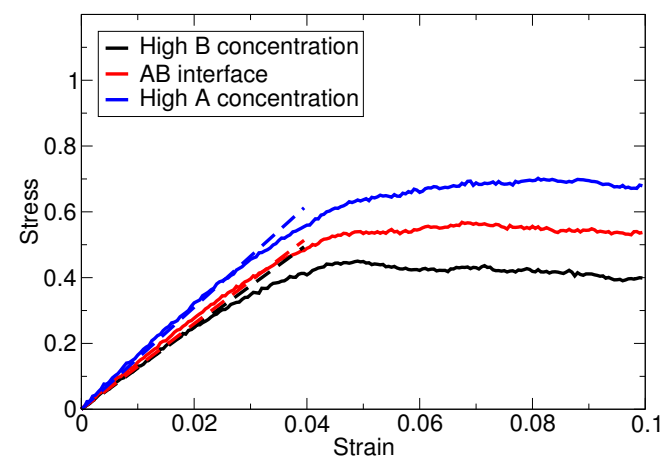

(d) $\varepsilon_{A A}=1.3$

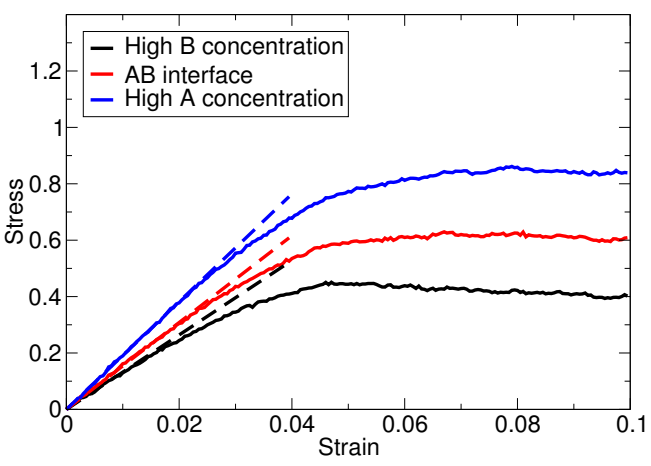

(f) $\varepsilon_{A A}=1.6$

Figure 3.17: Shear stress, regions: $k_{B} T=0.3$ and $k_{B} T=0.1$. The figures on the right side show stress for $k_{B} T=0.3$ and those on the left side for $k_{B} T=0.1$ for different $\varepsilon_{A A}$. The dashed lines represent the expected result for the shear stress in the linear regime. The shear stress measured while actually shearing the system is given by the solid line. Painting a more intricate picture than in figure 3.14, the shear stress is given for each of the three regions, high $\mathrm{A}$ concentration, $\mathrm{AB}$ interface, and high $\mathrm{B}$ concentration. 


\begin{tabular}{|l|l|l|l|l|l|l|}
\hline & \multicolumn{2}{|c|}{$A$-rich } & \multicolumn{2}{c|}{ Interface } & \multicolumn{2}{c|}{$B$-rich } \\
\hline$\varepsilon_{A A}$ & Tensor & NEMD & Tensor & NEMD & Tensor & NEMD \\
\hline & \multicolumn{5}{|c|}{$k_{B} T=0.3$} \\
\hline 1.1 & $13.2 \pm 2.9$ & $12.6 \pm 4.9$ & $12.5 \pm 0.7$ & $12.4 \pm 0.6$ & $12.5 \pm 3.2$ & $11.9 \pm 3.3$ \\
\hline 1.3 & $15.5 \pm 2.9$ & $15.8 \pm 2.1$ & $13.0 \pm 2.5$ & $13.8 \pm 0.7$ & $12.5 \pm 3.2$ & $12.5 \pm 0.8$ \\
\hline 1.6 & $19.1 \pm 1.2$ & $19.7 \pm 1.5$ & $15.4 \pm 1.2$ & $15.5 \pm 1.1$ & $13.2 \pm 1.8$ & $12.8 \pm 2.1$ \\
\hline & \multicolumn{5}{|c|}{$k_{B} T=0.1$} \\
\hline 1.1 & $16.7 \pm 4.0$ & $16.0 \pm 2.9$ & $15.5 \pm 1.0$ & $15.2 \pm 0.3$ & $16.0 \pm 4.0$ & $15.0 \pm 2.2$ \\
\hline 1.3 & $19.4 \pm 2.1$ & $17.8 \pm 2.3$ & $16.6 \pm 1.5$ & $16.5 \pm 1.1$ & $15.8 \pm 2.3$ & $15.0 \pm 0.6$ \\
\hline 1.6 & $22.9 \pm 1.5$ & $21.9 \pm 1.1$ & $18.0 \pm 1.4$ & $17.8 \pm 0.7$ & $15.8 \pm 2.1$ & $15.4 \pm 1.4$ \\
\hline
\end{tabular}

Table 3.7: Comparison of the shear modulus in $A$-rich regions, the interfacial regions, and $B$-rich regions as calculated from the local elastic modulus tensor with the shear modulus from NEMD simulations at $k_{B} T=0.3$ and $k_{B} T=0.1$. An $A$-rich box in the simulation volume contains at least ninety percent $A$ monomers, a $B$-rich box contains at least ninety percent $B$-monomers and the interface boxes fulfill neither condition.

\subsubsection{Correlation of Local Shear Modulus and Shear Stress}

After having looked at the influence the concentration of the different types of monomers in random block copolymer melts has on the shear and bulk modulus, we turn now to an analysis of the local elastic constants. We want to find a correlation for glassy polymer melts between the local shear modulus in the small boxes calculated from the elastic modulus tensor, as in equations (3.29), and the local shear stress when the system is sheared. We start with a homopolymer melt, $\varepsilon_{A A}=1.0$. Let us first look at the distribution of local shear moduli in the case of a homopolymer melt for $k_{B} T=0.1$ and $k_{B} T=0.3$. As we see in figure 3.18 , there are boxes with negative shear modulus. These are not unphysical as such [YPLdP05]. The distributions were fitted with Gaussian distributions, which resulted in the shear modulus, $G$, and its standard deviation, $\sigma_{G}$, in table 3.8 .

Now we go on to the correlation of local shear modulus from the elastic modulus tensor and the one calculated via the local shear stress. We follow the same procedure as in the previous subsection 3.4.1. For a measure of the correlation, we calculate the correlation coefficient of the $N_{b o x}$ measurements of $G$ and $G_{N E M D}$, using again the 
global strain with the local stress,

$$
R=\frac{\sum_{i=1}^{N_{b o x}}\left(G_{i}-\langle G\rangle\right)\left(G_{N E M D, i}-\left\langle G_{N E M D}\right\rangle\right)}{\sqrt{\sum_{i=1}^{N_{b o x}}\left(G_{i}-\langle G\rangle\right)^{2} \sum_{i=1}^{N_{b o x}}\left(G_{N E M D, i}-\left\langle G_{N E M D}\right\rangle\right)^{2}}}
$$

and calculate the $p$-value as an upper boundary of the probability of finding a distribution of values as in this measurement with no correlation between the quantities.

The results are in table 3.9. The results are very weakly correlated, so we are unable to reproduce the results by Yoshimoto et al. [Y $\left.\mathrm{YJVW}^{+} 04\right]$, who find a strong correlation, visualized by a scatter plot as in figure 3.19 . If there were a stronger correlation between the shear stress, equivalent to the shear modulus used for the calculation of the correlation coefficient, the ellipse in this figure would be tilted.

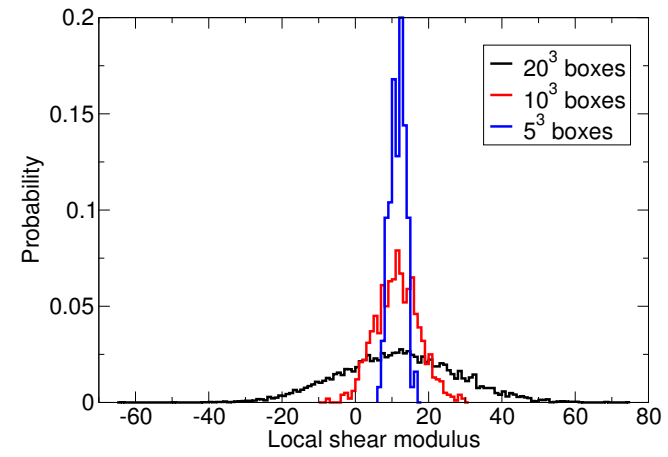

(a) $k_{B} T=0.3$

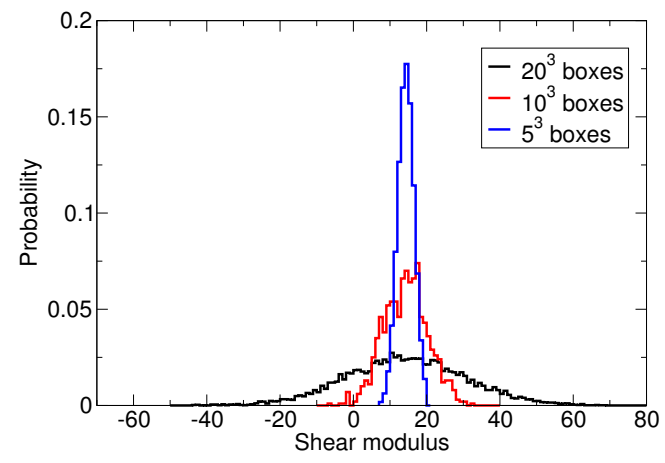

(b) $k_{B} T=0.1$

Figure 3.18: The distribution of the local shear modulus for different numbers of cubic boxes per simulation volume. For large numbers of boxes, which is equivalent to small boxes, we find negative values of the shear modulus. 


\section{Bulk Properties}

\begin{tabular}{|l|l|l|l|l|l|l|}
\hline & \multicolumn{3}{|c|}{$k_{B} T=0.3$} & \multicolumn{3}{c|}{$k_{B} T=0.1$} \\
\hline & $20^{3}$ boxes & $10^{3}$ boxes & $5^{3}$ boxes & $20^{3}$ boxes & $10^{3}$ boxes & $5^{3}$ boxes \\
\hline$G$ & 12.1 & 11.9 & 12.2 & 14.7 & 14.8 & 14.9 \\
\hline$\sigma_{G}$ & 15.9 & 5.9 & 2.3 & 16.2 & 6.1 & 2.3 \\
\hline
\end{tabular}

Table 3.8: Comparison of the fitted expectation value of the shear modulus, $G$, and its standard deviation, $\sigma_{G}$, according to the distribution of figure 3.18 for different numbers of boxes. A Gaussian distribution was used to fit the results.

\begin{tabular}{|l|l|l|l|l|l|l|}
\hline & \multicolumn{3}{|c|}{$k_{B} T=0.3$} & \multicolumn{3}{c|}{$k_{B} T=0.1$} \\
\hline & $20^{3}$ boxes & $10^{3}$ boxes & $5^{3}$ boxes & $20^{3}$ boxes & $10^{3}$ boxes & $5^{3}$ boxes \\
\hline$R$ & 0.022 & 0.049 & 0.013 & 0.055 & 0.073 & -0.031 \\
\hline$p$ & 0.050 & 0.125 & 0.882 & $<0.001$ & 0.02 & 0.730 \\
\hline
\end{tabular}

Table 3.9: Correlation between the local shear modulus as calculated from the elastic modulus tensor and the shear modulus calculated from NEMD simulations. $R$ is the correlation coefficient, while $p$ gives the upper boundary of the probability of finding this correlation with no correlation between the two observables. While $p$ is smaller than 0.05 for $k_{B} T=0.1$ and $20^{3}$ and $10^{3}$ boxes, the correlation is too small to call these quantities correlated. 


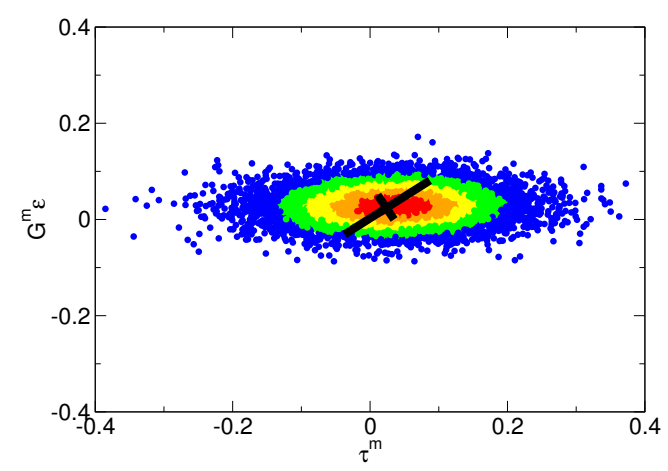

(a) $\epsilon_{4}=0.0025$

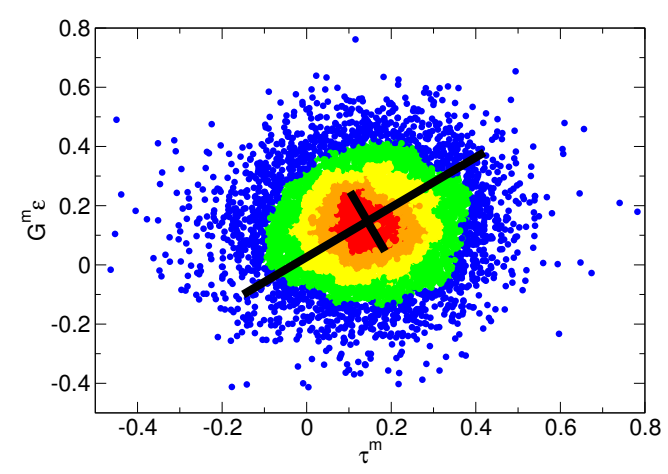

(b) $\epsilon_{4}=0.01$

Figure 3.19: Scatter plots of the measured shear stress, the abscissa, versus the expected shear stress from the local elastic modulus tensor, the ordinate. The temperature of the system is $k_{B} T=0.1$ and the simulation volume is subdivided into $20^{3}$ boxes. Each dot is for one box, while the coloring is used to indicate the density of the dots. Red indicates a high density, while blue means that the density of dots in the vicinity is low. The solid black lines drawn show the major and minor axis of an ellipse we would expect with a higher correlation between these two values. 



\title{
4 Selectively Attractive Substrate
}

\author{
"Cuiusvis hominis est errare, \\ nullius nisi insipientis \\ in errore perseverare"
}

(Marcus Tullius Cicero)

In this chapter, we examine the properties of a random block copolymer melt in contact with a selective substrate. The snapshots in figure 4.1 show the system for three different sets of parameters in MD and SCMF simulations. As for the bulk simulations, the agreement of the structure is clearly visible in the snapshots.

We start with an overview of previous work concerning the polymer-solid interfacial properties and theories, which predict structural properties of our system.

Subsequently, we discuss the potentials used, as well as structural and dynamical properties of the polymer-solid interface and look for signs of the interphase, which is one of the main goals of this work as described in the introduction, see chapter 1 The interphase is a region close to the substrate, which can span several $R_{\mathrm{e}}$, and in which the properties of the melt differ from the bulk and the interface. We continue with mapping the soft, coarse-grained model to the Lennard-Jones bead-spring model and observe the subsequent equilibration. In the end, we measure mechanical properties of the Lennard-Jones bead-spring model after quenching the melt below the glass transition temperature. 


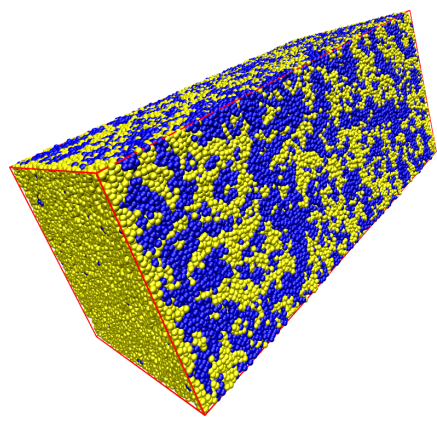

(a) $\varepsilon_{A A}=1.1$

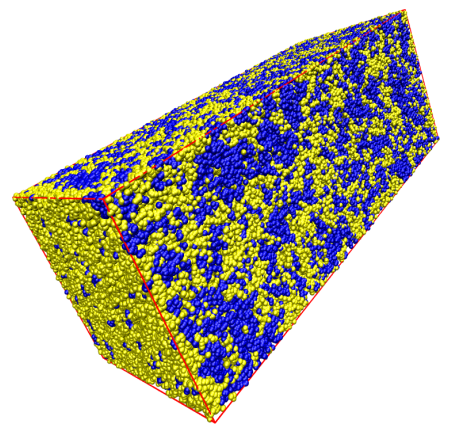

(d) $\chi_{0} N=30$

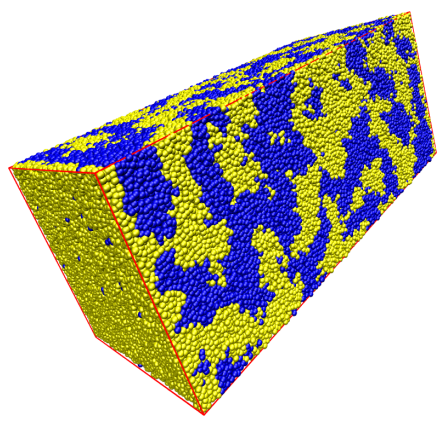

(b) $\varepsilon_{A A}=1.3$

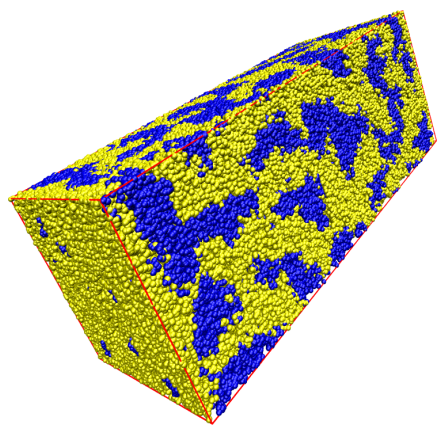

(e) $\chi_{0} N=100$

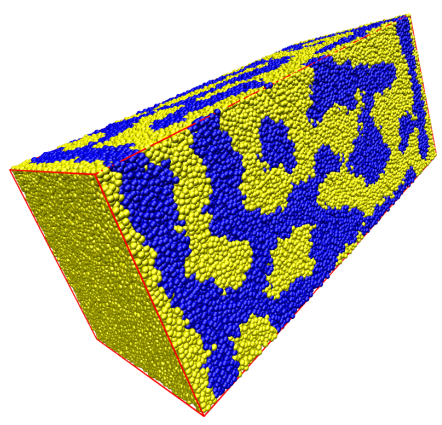

(c) $\varepsilon_{A A}=1.6$

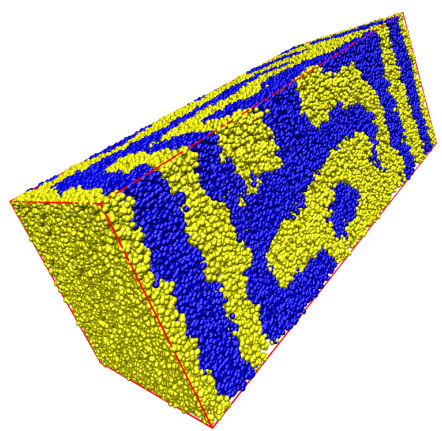

(f) $\chi_{0} N=240$

Figure 4.1: Snapshots for different values of the interaction parameter as indicated below each figure. The upper snapshots, (a) - (c), are from MD simulations while the lower figures, (d) - (f), show snapshots obtained by SCMF simulations. The substrates attract only $B$ monomers. As for the bulk simulations, see figure 3.1, the structures formed in contact with a substrate show a high similarity between the two different models. These figures were produced using VMD[HDS96].

\subsection{Theoretical Background}

The influence of contacts with substrates on random block copolymers has been studied for selectively attractive substrates using self consistent field equations [GC94]. Investigations in theory and simulation have probed the adsorption of random copolymer chains for a single chain $\left[\mathrm{BHM}^{+} 08\right]$, depending on the correlation of the sequence [PDS09], and for patterned substrates [SCB98, KKK06]. The influence of the sequence distribution of random copolymers on adsorption has been analyzed in a combined experimental and theoretical study by Jhon et al. [ $\left.\mathrm{JSG}^{+} 09\right]$. Experimentally, 


\subsection{Theoretical Background}

the adhesive properties and composition of random copolymers have been studied by Diethert et al. [DPWMB10]. SCMF simulations have been employed to simulate the influence of patterned substrates on structure formation for diblock copolymers $\left[\mathrm{SMK}^{+} 05, \mathrm{DMS}^{+}\right.$06, $\left., \mathrm{EMS}^{+} 07, \mathrm{DMS}^{+} 08\right]$ at these substrates.

For diblock copolymers, the ordering at the substrate when close to an order-disorder transition, has been investigated by Fredrickson [Fre87] who found a longer-range ordering at the substrate. Milner and Morse [MM96] managed to extract from the static structure factor of the composition of diblock copolymers in the bulk a prediction of the composition profile at the substrate.

For polymer glasses, Baschnagel and Binder [BB95] investigated the structural and dynamical properties of homopolymers which are influenced by a substrate. There they found signs of an interphase region.

Another interesting area of research is the limited resolution of coarse-grained models in contact with a wall, see Ramírez-Hernández et al. [RHDdP10] and Müller et al. [MSD $\left.{ }^{+} 11\right]$. The length scale of the interface region at the substrate is often on the order of or shorter than the typical resolution of coarse-grained models. Thus, the interfacial properties, for example the surface tension are not accurately reproduced by a coarse-grained model. In these papers, a modification of either the bonded interaction or the wall-potential is proposed to fix the discrepancies in surface tension and chain end density caused by the length scale of the discretization of a coarse-grained polymer model in contact with a wall.

\subsubsection{Silberberg's Argument}

The change of polymer conformations in a melt when confined by a reflecting wall was first treated by Silberberg [Sil82]. The idea behind his reasoning is that the conformation of chains near a wall can be derived from the properties of the bulk. We take a bulk polymer melt, and put a virtual plane into its middle. This virtual plane acts as a wall, therefore chains are not allowed to cross it. To this end, we change the chain conformations of each chain crossing this virtual plane. To describe this process, one should first designate one chain end as start and the other one as termination. The start is the chain end which is closer to the wall. Chains are now assigned to the side of the wall, where the starting end resides. The part of the chain which is on the other side is then reflected in the plane, so it is situated on the same side of the wall as the starting end. In this way, the conformation in the direction perpendicular to the wall 


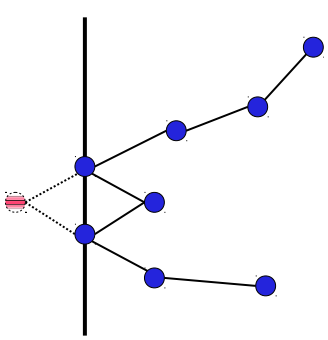

(a)

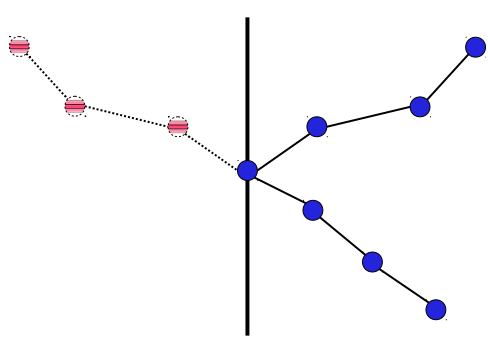

(b)

Figure 4.2: Conformations at wall. These two sketches show the change of the conformations by the virtual wall. Chains crossing the wall are folded to the side of the wall where the chain end is situated which is closest to the wall.

changes, while the density remains constant on average. This argument only holds for a non-interacting wall, and is only true in the case that the presence of the wall does not change the chain end distribution.

Assuming that the chain is Gaussian, Silberberg resorted to random walk statistics to solve this problem analytically. Since the virtual wall is flat, the problem is reduced to one dimension, the direction perpendicular to the wall. The number of chains in the bulk with $N$ segments, which start at $x_{0}$ and terminate at $x_{N}$ can be approximated by

$$
\Omega\left(x_{0}, x_{N}, N\right) \simeq \Omega^{\text {Tot }} \frac{2}{b \sqrt{2 \pi}} \exp \left(-\frac{\left(x_{N}-x_{0}\right)^{2}}{2 R_{\mathrm{eo}}^{2}}\right)
$$

where $\Omega^{\text {Tot }}$ is the total number of chains which start at $x_{0}$. The mean squared end-toend distance then yields the expected result of

$$
<R_{e}^{2}>=\int_{x_{0}}^{\infty} d x_{N}\left(x_{0}-x_{N}\right)^{2} \frac{2}{R_{\mathrm{eo}} \sqrt{2 \pi}} \exp \left(-\frac{\left(x_{N}-x_{0}\right)^{2}}{2 R_{\mathrm{eo}}{ }^{2}}\right)=R_{\mathrm{eo}}{ }^{2} .
$$

After reflecting the chains as described above, the number of chains to be found on the selected side of the virtual wall and starting at position $x$ is

$$
\begin{aligned}
\Omega^{\prime}\left(x_{0}=x, x_{N}, N\right)= & \frac{2 \Omega^{\text {Tot }}}{R_{\mathrm{eo}} \sqrt{2 \pi}}\left[\int_{x}^{\infty} d x_{N} \exp \left(-\frac{\left(x_{N}-x\right)^{2}}{2 R_{\mathrm{eo}}^{2}}\right)\right. \\
& \left.+\int_{-\infty}^{-x} d x_{N} \exp \left(-\frac{\left(x_{N}-x\right)^{2}}{2 R_{\mathrm{eo}}^{2}}\right)\right] \\
= & \Omega^{\text {Tot }}\left(2-\operatorname{erf}\left(2 \frac{x}{\sqrt{2} R_{\mathrm{eo}}}\right)\right) .
\end{aligned}
$$


where $\operatorname{erf}(x)$ is the error function. The number of chains terminating at position $x$ can be determined similarly as

$$
\Omega^{\prime}\left(x_{0}, x_{N}=x, N\right)=\Omega^{\text {Tot }} \operatorname{erf}\left(2 \frac{x}{\sqrt{2} R_{\mathrm{eo}}}\right) .
$$

Therefore, the introduction of a wall into the bulk melt does not change the local chain end density. We now want to look at the $x$-component of the mean squared end-to-end distance, $<R_{e}^{2}>{ }_{x}$. For this quantity, the sum of two integrals has to be evaluated,

$$
<R_{e}^{2}>_{x}=\frac{1}{2} \frac{2}{R_{\mathrm{eo}} \sqrt{2 \pi}}\left(I_{1}+I_{2}\right) .
$$

The factor $\frac{1}{2}$ stems from the fact that the calculation is performed for chain terminations and starts at $x$, so this is needed to counter the double-counting. The integrals are

$$
I_{1}=\int_{0}^{\infty} d x_{N}\left(x_{N}-x\right)^{2} \exp \left(-\frac{\left(x_{N}-x\right)^{2}}{2 R_{\mathrm{eo}}{ }^{2}}\right)
$$

for chains which start or terminate at $x$ and whose chain terminations are not affected by the wall,

$$
I_{2}=\int_{0}^{\infty} d x_{N}\left(x_{N}-x\right)^{2} \exp \left(-\frac{\left(x_{N}+x\right)^{2}}{2 R_{\mathrm{eo}}{ }^{2}}\right)
$$

for chains which start or terminate at $x$ and where the chain termination was on the other side of the wall.

The calculation of these integrals gives as a result

$$
\begin{aligned}
\frac{\left\langle R_{e}^{2}\right\rangle_{x}}{\left\langle R_{e}^{2}>\right.}= & {\left[1-4 \frac{x}{\sqrt{2} R_{\mathrm{eo}}} \frac{1}{\sqrt{\pi}} \exp \left(-\frac{x^{2}}{2 R_{\mathrm{eo}}{ }^{2}}\right) \ldots\right.} \\
& \left.+4 \frac{x^{2}}{2 R_{\mathrm{eo}}{ }^{2}}-4 \frac{x^{2}}{2 R_{\mathrm{eo}}{ }^{2}} \operatorname{erf}\left(\frac{x}{\sqrt{2} R_{\mathrm{eo}}}\right)\right] .
\end{aligned}
$$

\subsubsection{Linear Response Theory}

In this subsection, we look at the theoretical predictions for composition fluctuations of a random block copolymer melt in contact with a solid substrate. The idea of linear response theory in this case is to couple a local perturbation, a wall potential, to the composition profile. Since we know the static structure factor of the composition in the bulk, which is the Fourier transform of the composition profile, we know how this perturbation propagates in momentum space. This knowledge can be transformed back into real space, via a discrete Fourier transform, and gives us a composition 


\section{Selectively Attractive Substrate}

profile. We start our calculations with a work by Milner and Morse [MM96]. In this paper, they derived a response of a symmetric diblock copolymer melt to a selectively attractive wall potential. When describing a polymer melt confined by two substrates in the $\mathrm{x}$-direction, one can assume an isotropic behaviour in the directions parallel to the substrate, thus the problem is reduced to one dimension. The concentration profile of the melt can be expanded in a Fourier series, decoupling the parallel and the perpendicular coordinates:

$$
c\left(x, \vec{r}_{\|}\right)=\frac{1}{A d} \sum_{k_{x}, \vec{k}_{\|}} \tilde{c}\left(k_{x}, \vec{k}_{\|}\right) f_{k_{x}, \vec{k}_{\|}}\left(x, \vec{r}_{\|}\right),
$$

with $A$ being the area of the substrate, $d$ the separation of the substrates, $c(\vec{r})=$ $\left[\Phi_{A}-\Phi_{B}\right](\vec{r})$ is the concentration difference between $A$ and $B$ monomers in real space, $\tilde{c}(\vec{k})$ its Fourier transform, and

$$
f_{k_{x}, \vec{k}_{\|}}\left(x, \vec{r}_{\|}\right)=\sqrt{2} \exp \left(i \vec{k}_{\|} \vec{r}_{\|}\right) \cdot \cos \left(k_{x} x\right)
$$

where the $\vec{k}_{\|}$are arbitrary, two-dimensional vectors and $k_{x}=\pi m / d$, where $m$ is a positive integer. We are working in the regime $f=0.5$, which is the reason for $m \neq 0$, and the derivative with respect to $x$ at the boundaries vanishes, since we assume reflecting boundary conditions for a melt confined between two substrates. Now we proceed to derive the influence of the substrate on the concentration. The general expression for this coupling is the change in free energy due to the influence of the substrate

$$
\begin{aligned}
F_{\text {subs }} & =-\int d^{3} r c(\vec{r}) \cdot U_{\text {subs }}(\vec{r}) \\
& =-\int d x d^{2} r_{\|} c\left(x, \vec{r}_{\|}\right) \cdot U_{\text {subs }}(x)
\end{aligned}
$$

where the potential only depends on the distance from the substrate. With equations 4.11 and 4.12, this transforms into

$$
F_{\text {subs }}=-\frac{\sqrt{2}}{A d} \sum_{k_{x}, \vec{k}_{\|}} \tilde{c}\left(k_{x}, \vec{k}_{\|}\right) \int_{0}^{d} d x \cos \left(k_{x} x\right) U_{\text {subs }}(x) \int d^{2} r_{\|} \exp \left(i \vec{k}_{\|} \vec{r}_{\|}\right) .
$$

The potential we use in the SCMF simulations is proportional to $\exp \left(-\left(x / x_{0}\right)^{2} / 2\right)$, therefore an analytical solution for the integral above is only possible if the simulation box extends to infinity. This is not possible, however, the length scale of the potential, 


\subsection{Theoretical Background}

$x_{0}$, is $0.15 R_{\mathrm{eo}}$, which lets us approximate the integral over $x$ by its limit for $d \rightarrow \infty$ with only a very small error. Substituting the potential with

$$
U_{\text {subs }}(x)=\sqrt{\pi / 2} x_{0} g N \cdot \exp \left(-\left(x / x_{0}\right)^{2} / 2\right)
$$

where $g N$ is the interaction strength of the substrate, in equation 4.14 and evaluating the integral over $\mathrm{x}$, yields

$$
F_{\text {subs }}=-\frac{\sqrt{2}}{A d} \sum_{k_{x}, \vec{k}_{\|}} \tilde{c}\left(k_{x}, \vec{k}_{\|}\right) g \exp \left(-\left(k_{x} x_{0}\right)^{2} / 2\right) \int d^{2} r_{\|} \exp \left(i \vec{k}_{\|} \vec{r}_{\|}\right)
$$

The integral over the plane wave in the plane parallel to the substrate is zero when $\vec{k}_{\|} \neq 0$, the whole equation reduces to

$$
F_{\text {subs }}=-\frac{\sqrt{2}}{d} \sum_{k_{x}} \tilde{c}\left(k_{x}, 0\right) g \exp \left(-\left(k_{x} x_{0}\right)^{2} / 2\right) .
$$

The real space response of the melt to this potential is then the Fourier transform of the product of the compositional structure factor and the potential in momentum space

$$
c(x, 0)=\frac{2}{d} \sum_{k_{x}} \cos \left(k_{x} x\right) S\left(k_{x}\right) g \exp \left(-\left(k_{x} x_{0}\right)^{2} / 2\right)
$$

where $S\left(k_{x}\right)$ is the compositional structure factor introduced in chapter 3 , see equation (3.1). Milner and Morse used a simpler potential

$$
U_{s u b s, \delta}(x)=U_{0, \delta} \delta(x)
$$

in [MM96] with a potential strength $U_{0, \delta}$, which simplifies the calculation and has the following results:

$$
F_{\text {subs }}=-\frac{\sqrt{2}}{d} \sum_{k_{x}} \tilde{c}\left(k_{x}, 0\right) U_{0, \delta}
$$

and

$$
c(x, 0)=\frac{2}{d} \sum_{k_{x}} \cos \left(k_{x} x\right) S\left(k_{x}\right) U_{0, \delta} .
$$

We use this theory later to compare the predictions from the static structure factor of the composition with actual simulation results. 


\subsection{Properties of the Interface and Interphase}

In this section, we describe the systems used to simulate a random block copolymer melt in contact with a solid substrate and elaborate on their properties. In particular, we find a region which extends several $R_{e}$ from the substrate and shows properties different from the bulk system. This region, called the interphase, will be in the focus of this description together with the interface between polymer melt and solid substrate.

\subsubsection{Wall Potential}

The solid wall in the SCMF model is implemented by breaking the periodic boundary condition in one direction. These boundaries are supplanted by a hard wall which means that movement through these boundaries is no longer possible. In addition, to simulate the selectively attractive substrate, we modify the non-bonded interaction, equation 2.39], by a potential [DMS ${ }^{+} 08$ ]

$$
\mathcal{H}_{n b, \text { wall }}=\mathcal{H}_{n b}+\rho_{c} \int d^{3} r U_{\text {wall }}(\vec{r})\left(\hat{\phi}_{A}-\hat{\phi}_{B}\right) .
$$

Since coarse-grained models are not able to resolve the structure of solid substrates below their coarse-graining scale, [RHDdP10, $\left.\mathrm{MSD}^{+} 11\right]$, we choose a soft potential that only depends on the distance from the substrate

$$
U_{\text {wall }}(\vec{r})=\frac{g N R_{\mathrm{eo}}}{r_{\text {wall }}} \exp \left(-\frac{x^{2}}{2 r_{\text {wall }}^{2}}\right) .
$$

This potential is short-ranged, $r_{\text {wall }}=0.15 R_{\text {eo }}$, soft, and selectively attractive to one type of monomers. For a positive value of $g N$, it attracts monomers of type $B$ while repelling monomers of type $A$, and vice versa for negative values of $g N$.

For the Lennard-Jones model, we follow a similar strategy. Again, one direction of the simulation volume is no longer treated with periodic boundary conditions. Then we implement a wall of Lennard-Jones particles, which interacts differently with the two different types of monomers. We do not use explicit Lennard-Jones particles, but integrate over the whole substrate for a potential which is, as for SCMF, only dependent on the distance from the substrate. With the Lennard-Jones potential

$$
U_{L J}(r)=4 \varepsilon_{\text {wall }}\left(\left(\frac{\sigma}{r}\right)^{12}-\left(\frac{\sigma}{r}\right)^{6}\right)
$$


we are interested in the potential at a distance $x$ from the wall

$$
\begin{aligned}
U_{L J, 9-3}(x) & =\frac{4 \varepsilon_{\text {wall }}}{V_{p, \text { wall }}} \int_{x}^{\infty} d x^{\prime} \int_{0}^{\infty} d \rho \int_{0}^{2 \pi} d \phi \rho\left(\frac{\sigma^{12}}{\left(\rho^{2}+x^{\prime 2}\right)^{6}}-\frac{\sigma^{6}}{\left(\rho^{2}+x^{\prime 2}\right)^{3}}\right) \\
& =\frac{2 \pi \sigma^{3}}{3 V_{p, \text { wall }}} \varepsilon_{\text {wall }}\left(\frac{2}{15} \frac{\sigma^{9}}{x^{9}}-\frac{\sigma^{3}}{x^{3}}\right)
\end{aligned}
$$

where $V_{p, \text { wall }}$ is the volume of the unit cell of the wall's lattice. We set $V_{p, \text { wall }}$ to $\frac{2 \pi \sigma^{3}}{3}$ which is equal to a packing fraction of $\sqrt{2^{-3}} \approx 0.35$, close to the one of a diamond lattice, which is 0.34 [AM76]. Thus, the potential we use to simulate a substrate for the Lennard-Jones particles is

$$
U_{L J, w a l l}(x)=\varepsilon_{\text {wall }}\left(\frac{2}{15} \frac{\sigma^{9}}{x^{9}}-\frac{\sigma^{3}}{x^{3}}\right),
$$

also called the Lennard-Jones $9-3$ potential. Here the selectivity of the potential is not achieved through different interaction parameters, but the cutoff. For the substrate to be attractive, we chose a cutoff of $r_{c}=2.5 \sigma$, when we want it to be repulsive, we choose $r_{c}=0.715 \sigma$.

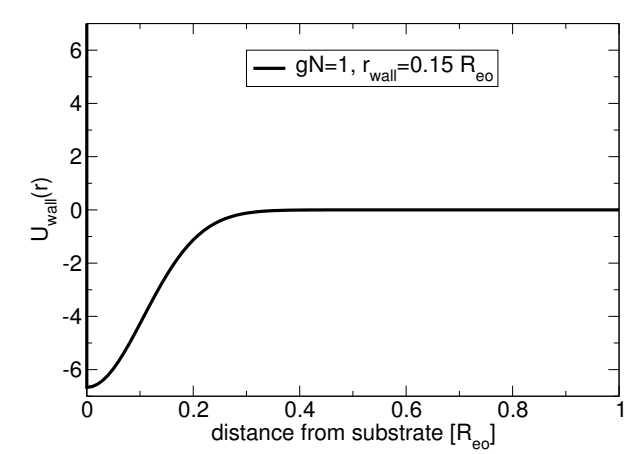

(a)

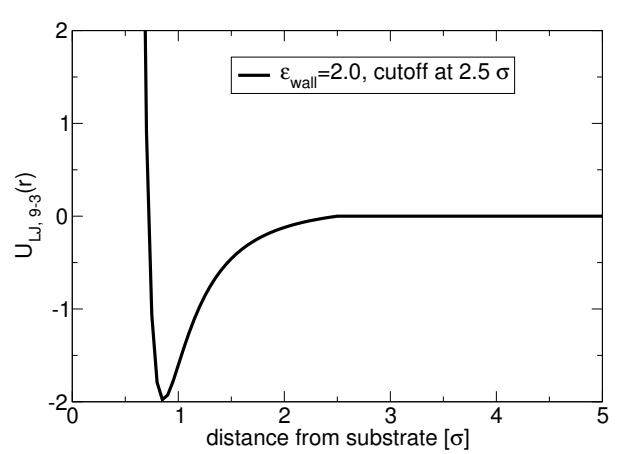

(b)

Figure 4.3: Potentials of the substrates. The two potentials for (a) SCMF and (b) MD simulations are plotted on the length scale of the simulation program. The length scale is $1 R_{\text {melt }}=10 \sigma=1.2 R_{\mathrm{eo}}$. 


\subsubsection{Composition Profile}

Now we want to find parameters for the interaction strength, $g N$ and $\varepsilon_{\text {wall }}$ which lead to a complete wetting of the preferred species. To this end, we look at the volume fraction as a function of the distance from the substrate. The procedure for SCMF simulations is the following. First we generate a starting configuration, using a homopolymer melt, $\alpha=0, \chi_{0} N=0, \kappa N=600$, in a box of dimension $8 \cdot 8 \cdot 16 R_{\mathrm{eo}}^{3}$, with 6 grid points per $R_{\mathrm{eo}}$. In the largest dimension, we implement a hard wall at the boundary, the other two dimensions have periodic boundary conditions. The simulation volume is filled with 8320 chains of 120 monomers each, and simulated for $1.5 \cdot 10^{5}$ time steps using smart Monte Carlo simultaneously employing the quasi-instantaneous field approximation.

We use this equilibrated homopolymer melt as the starting configuration for the random block copolymer in contact with a solid substrate. The parameters of these simulations are $\chi_{0} N=240$ with $\alpha=0.05, \chi_{0} N=100$ with $\alpha=0.02$, and $\chi_{0} N=30$ with $\alpha=0.01$. For the search of interesting systems, we choose

$$
g N \in\{-10 ;-5 ;-1 ;-0.5 ; 0 ; 0.5 ; 1 ; 5 ; 10\} .
$$

The simulation is then run for $6 \cdot 10^{5}$ time steps, after which we compare the volume fraction profile with the predictions of linear response theory, see figure 4.5 . For large enough distance between the attractive substrates, as used in our simulations, the difference between the two approaches, a short-range or a delta-function potential, for the linear response theory is negligible, as can be seen in figure 4.4. This becomes clear when we consider which values of $q$ contribute when we use a short-range-potential. These values of $q$ are much larger than the region of the peak, therefore their influence on the actual composition is very small. We average over the composition profiles of the two substrates in the simulation box. The choice of $g N=-1$ for substrates attracting $A$ monomers and $g N=1$ for substrates attracting $B$ monomers generates a composition profile which is very similar to the results of the linear response theory. In the disordered case, $\chi_{0} N=30$ in figures $4.5(\mathrm{a})$ and $4.5(\mathrm{~b})$, there is no long-range ordering of the melt. For $\chi_{0} N=100$ and $\chi_{0} N=240$, see figures 4.5(c) to 4.5(f), the behaviour is markedly different. The composition profiles in both cases exhibit a long-range order extending more than $2 R_{e}$ from the substrate. The composition profiles show oscillations which decay exponentially. This is a sign of the interphase, a longer-range ordering induced by the substrate, which extends further into the simulation volume than the interfacial order which is seen for the disordered state in figures 


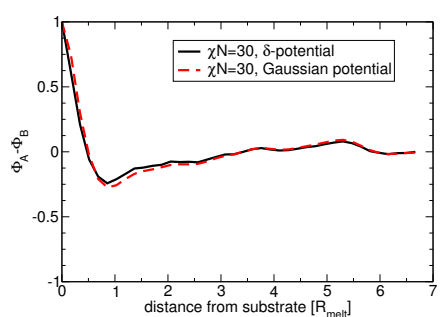

(a)

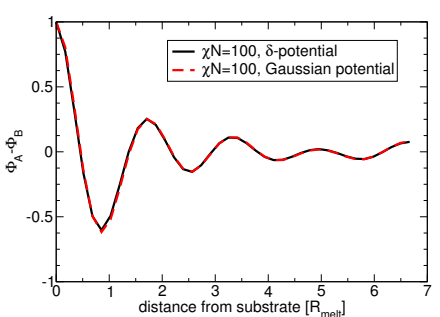

(b)

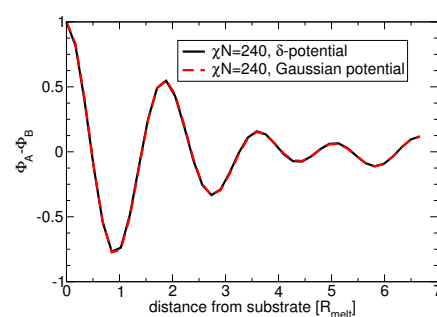

(c)

Figure 4.4: Linear response theory: potential shape. In these three figures the results for the linear response theory with a $\delta$-potential are compared with a Gaussian one. There are differences in Figure (a), with $\chi_{0} N=30, U_{0, \delta}=0.41$, and $g=0.5$, but in (b) with $\chi_{0} N=100, U_{0, \delta}=0.14$, and $g=0.16$ and (c) with $\chi_{0} N=240, U_{0, \delta}=0.08$, and $g=0.09$ there is no discernible difference between the results. We use the structure factors from figure 3.3 together with the linear response calculations in subsection 4.1.2.

\section{5(a) and 4.5(b).}

The simulation procedure for MD is very similar. Again, we start with a homopolymer melt which is confined in one direction by solid substrates, with periodic boundary conditions in the other two dimensions. The starting size of the simulation box is $136 \cdot 68 \cdot 68 \sigma^{3}$, with 8320 homopolymer chains of 60 monomers each placed randomly into the simulation volume. We start with a $N V E$ simulation over 100 time steps, where the maximal movement of a monomer is restricted to $0.05 \sigma$ per time step. We use this restriction to prevent the system from "blowing up". At the same time we ramp up the temperature linearly from $k_{B} T=0$ to $k_{B} T=1$. The wall potential is made repulsive by using a cutoff of $0.715 \sigma$ for the Lennard-Jones $9-3$ potential from equation (4.26). We proceed with a $N V T$ simulation at $k_{B} T=1$ for $10^{6}$ time steps after which we let the system equilibrate in a $N P_{t} T$ simulation at the same temperature and $P_{t}=0$ for $10^{7}$ time steps. The resulting configuration is then used as a starting configuration for the random block copolymer melt simulation.

The simulation of the random block copolymers continues in the $N P_{t} T$ ensemble at $k_{B} T=1$ and $P_{t}=0$ for $3 \cdot 10^{7}$ steps. We perform simulations with an attractive wall potential for $A$ monomers and for $B$ monomers. Starting with a value of $\varepsilon_{\text {wall }}=2.0$ we found it to be sufficient for a complete wetting of the substrate by the attracted type 


\section{Selectively Attractive Substrate}

of monomers in the case of $\varepsilon_{A A}=1.1$, see figures 4.6(a) and 4.6(b), and $\varepsilon_{A A}=1.3$, see figures 4.6(c) and 4.6(d). For $\varepsilon_{A A}=1.6$ this holds only when the substrate is attractive to $B$ monomers, see figures $4.6(\mathrm{e})$ and $4.6(\mathrm{f})$. Thus we decided to use a stronger interaction in the case of $\varepsilon_{A A}=1.6, \varepsilon_{\text {wall }}=3.0$. Again, the linear response theory gives us a good prediction of the composition profile close to the substrate. Furthermore, the interphase is clearly visible as well.

We can compare these results to experiments concerning diblock copolymer melts in contact with a selective substrate. If the temperature is below the value for orderdisorder transition in the bulk, diblock copolymers in experiments arrange themselves to form a lamellar phase, where the lamellæ is parallel to the substrate [ $\mathrm{MJR}^{+}$93]. This behaviour is observed in a mean-field approach with a Ginzburg-Landau free energy functional, too [TA01]. The distance between the interacting substrates has a strong influence on the temperature at which the order-disorder transition takes place [SAK ${ }^{+}$08], it rises for smaller distances. For temperatures above the order-disorder transition, linear response theory predicts exponentially decaying concentration oscillations [MM96] which has also been observed in experiments [ $\left.\mathrm{MRH}^{+} 97\right]$. 


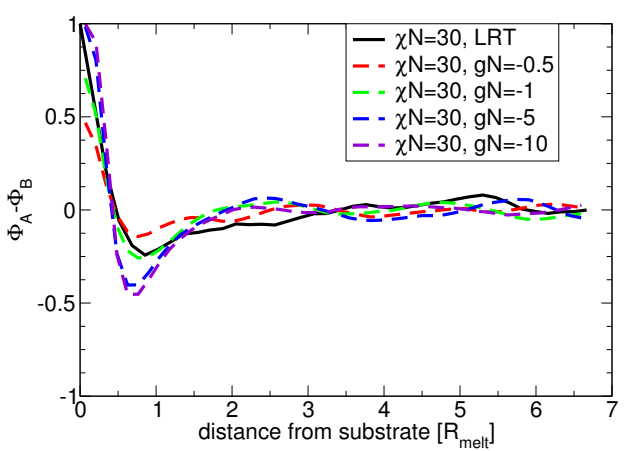

(a) $A$ monomers, $\chi_{0} N=30$

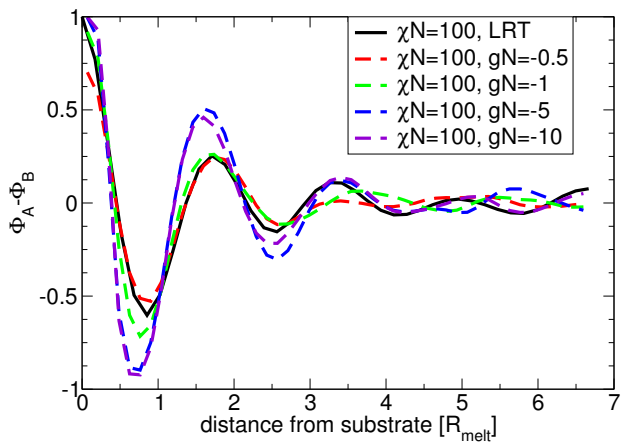

(c) $A$ monomers, $\chi_{0} N=100$

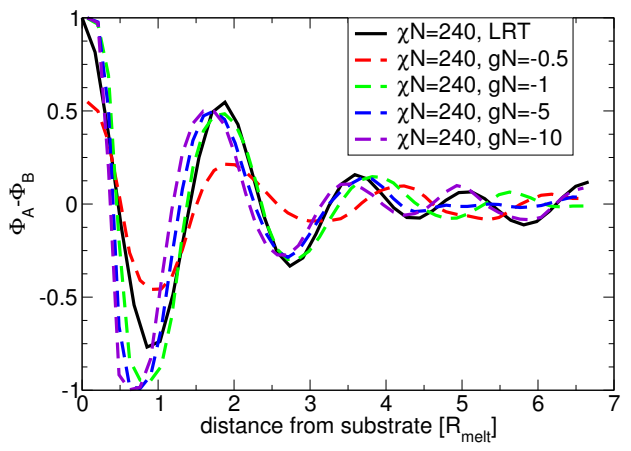

(e) $A$ monomers, $\chi_{0} N=240$

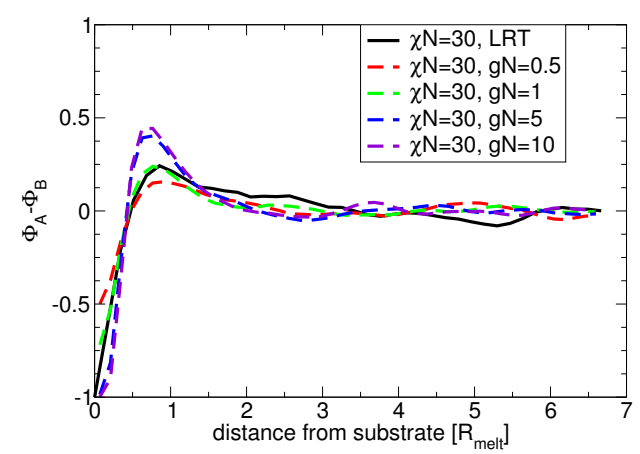

(b) $B$ monomers, $\chi_{0} N=30$

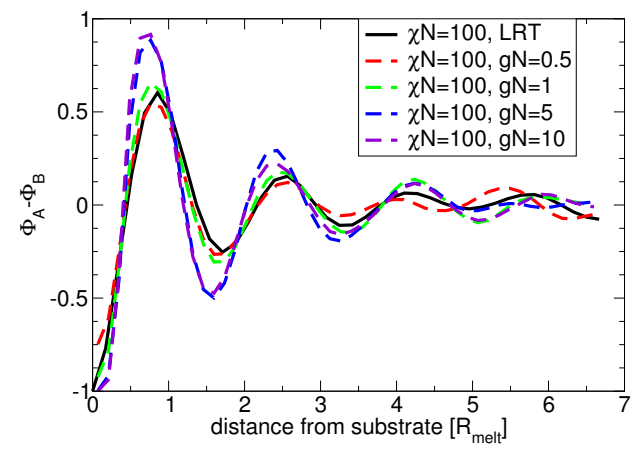

(d) $B$ monomers, $\chi_{0} N=100$

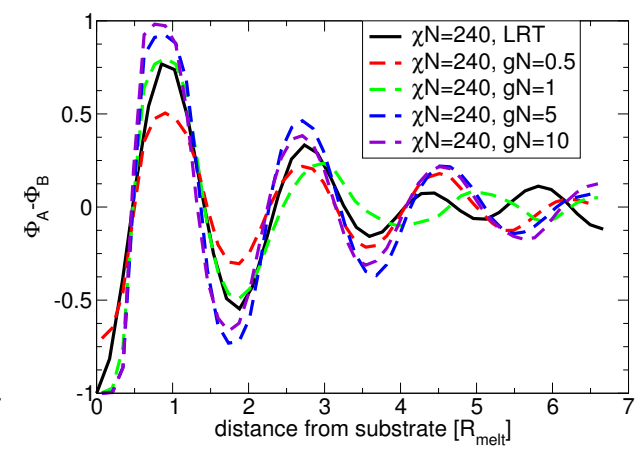

(f) $B$ monomers, $\chi_{0} N=240$

Figure 4.5: Structure of melt at substrate: SCMF simulations. These six figures show the results for the linear response theory with a $\delta$-potential, compared with SCMF simulations confined by a selectively attractive substrate for - from top to bottom $-\chi_{0} N=30, \chi_{0} N=100, \chi_{0} N=240$. The left figures show a substrate which is attractive to $A$ monomers while the right figures show a substrate attracting $B$ monomers. In all cases, $g N= \pm 1$ for the simulation results in a composition that is very similar to the results of the linear response theory. 


\section{Selectively Attractive Substrate}

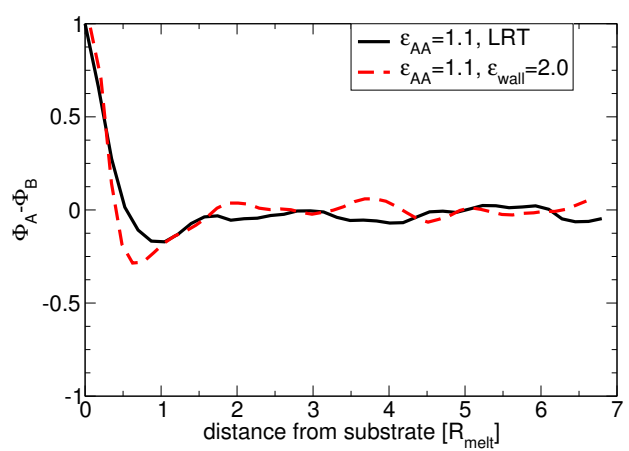

(a) $A$ monomers, $\varepsilon_{A A}=1.1$

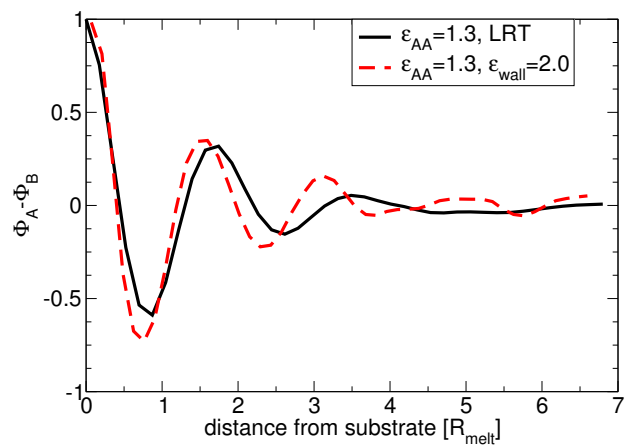

(c) $A$ monomers, $\varepsilon_{A A}=1.3$

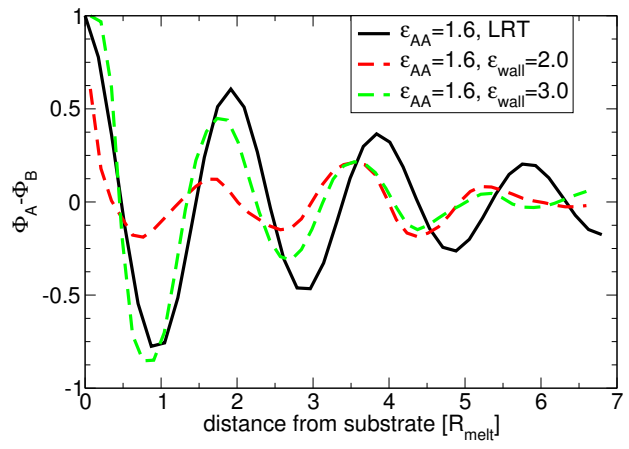

(e) $A$ monomers, $\varepsilon_{A A}=1.6$

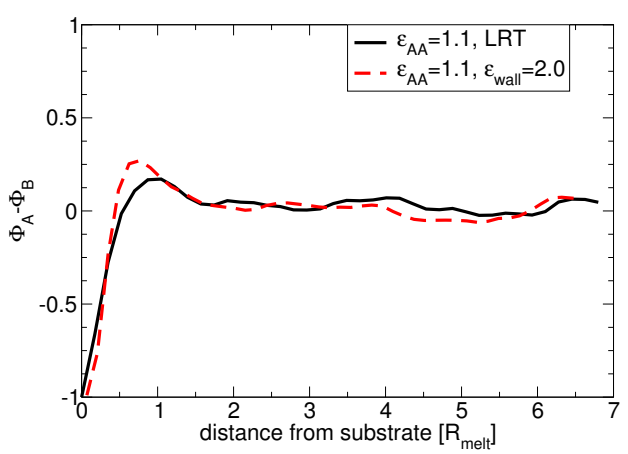

(b) $B$ monomers, $\varepsilon_{A A}=1.1$

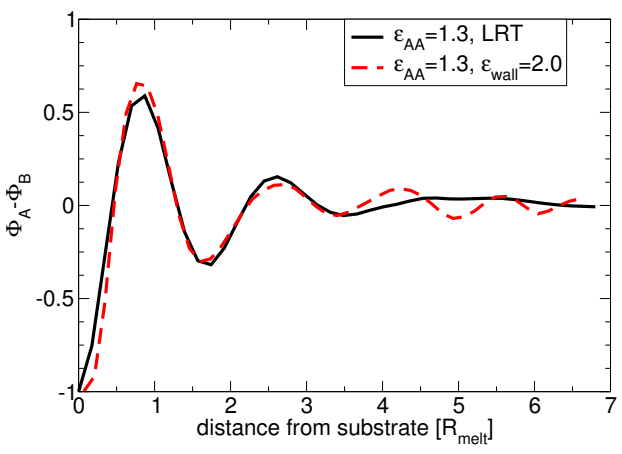

(d) $B$ monomers, $\varepsilon_{A A}=1.3$

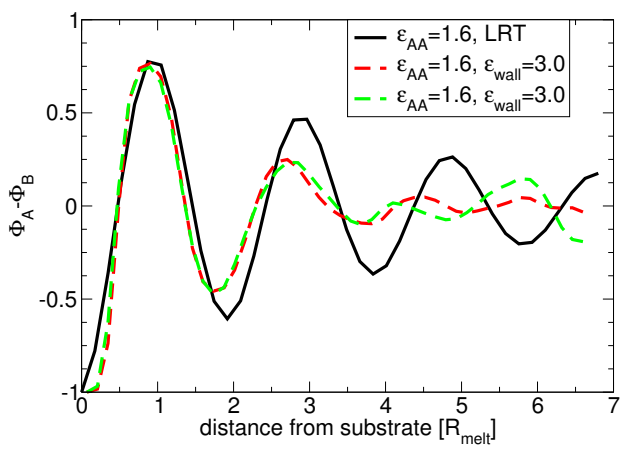

(f) $B$ monomers, $\varepsilon_{A A}=1.6$

Figure 4.6: Structure of melt at substrate: MD simulations. In these six figures the results for the linear response theory with a $\delta$-potential are compared with MD simulations confined by a selectively attractive substrate for - from top to bottom $-\varepsilon_{A A}=1.1$ and $\varepsilon_{\text {wall }}=2.0, \varepsilon_{A A}=1.3$ and $\varepsilon_{\text {wall }}=2.0$, and $\varepsilon_{A A}=1.6$ and $\varepsilon_{\text {wall }}=2.0$ and 3.0. The left figures show a substrate which is attractive to $A$ monomers while the right figures show a substrate attracting $B$ monomers. The composition of the MD simulation is similar to the linear response theory, for $\varepsilon_{A A}=1.6$ and an $A$-attractive substrate, we require a higher value of $\varepsilon_{\text {wall }}$ for a wetting of the substrate. 


\subsubsection{Segregation at the Substrate}

The composition profile describes where the different monomers can be found. Now we proceed to a coarser level, namely looking at the distribution of the chains themselves. The large number of different sequences, see equation (2.4), means that only comparably few chains of each sequence exist in the melt. Thus, trying to salvage information from the spatial distributions of each and every sequence is a daunting task. For this reason, we group the chains according to the number of $A$ blocks they contain, leaving us with only 7 different groups to look at. The homopolymers constitute two of these groups, leading to worse statistic in their cases. We sample the densities according to the position of the monomers, not the chains' centers of mass.

In the disordered case, the distribution of chains has been addressed by Kłos et al., [KRS10]. Their results for the distribution of $A$ homopolymers in a random block copolymer melt at a selective substrate agrees well with the disordered melt at $\chi_{0} N=$ 30 in figures 4.7(a) and 4.7(c). In the disordered melt, the segregation of the chains is very similar to the composition profile in figures 4.5(a) and 4.5(b), The polymers consisting mainly of the attracted type of monomer are enriched at the substrate, then depleted at a distance of $1 R_{e}$. For the polymers consisting mainly of the repulsed monomers, it is the other way around, while the polymers containing the same number of $A$ and $B$ monomers are depleted in the interface region. The asymmetry parameter is small, $\alpha=0.01$, so we do not expect and do not find a large deviation between $A$-attractive substrates, figure 4.7(a), and $B$-attractive substrates, figure 4.7(c), for the same absolute interaction strength of the substrate.

When we look at a higher incompatibility, $\chi_{0} N=100$, the behaviour near the substrate changes drastically. For a wall potential of $g N= \pm 1$, see figures $4.7(\mathrm{~d})$ and 4.7(f), we see composition fluctuations extending $3 R_{\text {melt }}$ into the bulk. This result is similar to the pure composition fluctuations we observed in figures 4.5(c) and 4.5(d). The ordering in the interphase therefore is not only relevant for the concentration of the monomers, but strongly influences the distribution of the chains as well. The slight asymmetry between the monomers, $\alpha=0.02$, shows itself in the sequence profiles.

For the highest value of the incompatibility we analyze, $\chi_{0} N=240$, the influence of the selectively-attractive substrate becomes even more pronounced. The ordering extends so far from the substrate that we suspect an interference between the both substrates. It extends at least $4 R_{\text {melt }}$ into the bulk for $g N= \pm 1$, see figures $4.7(\mathrm{~g})$ and 4.7(i). 


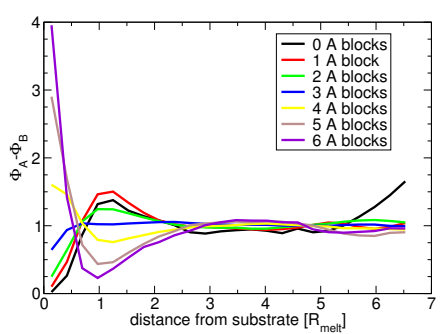

(a) $g N=-1, \chi_{0} N=30$

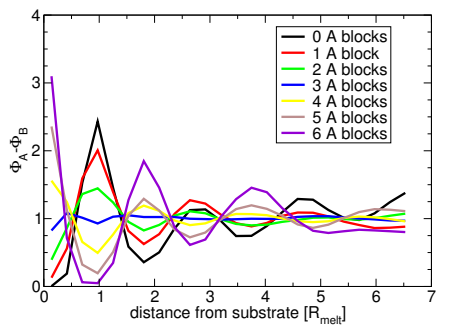

(d) $g N=-1, \chi_{0} N=100$

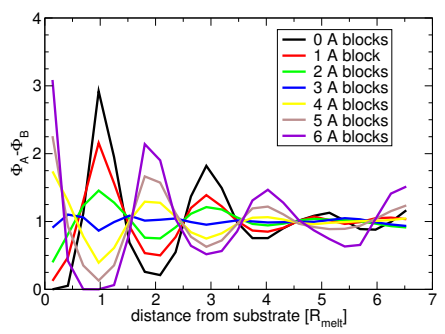

(g) $g N=-1, \chi_{0} N=240$

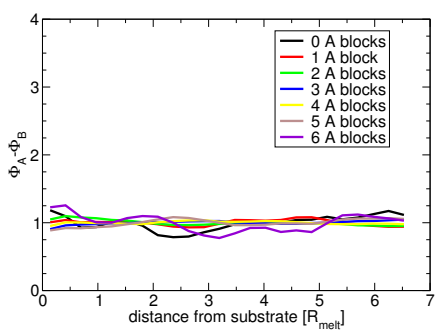

(b) $g N=0, \chi_{0} N=30$

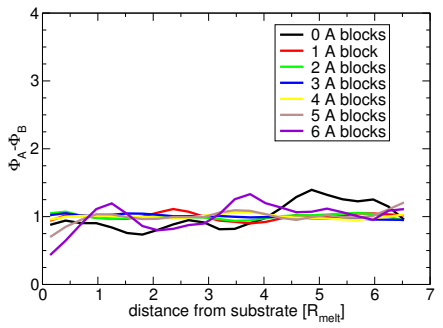

(e) $g N=0, \chi_{0} N=100$

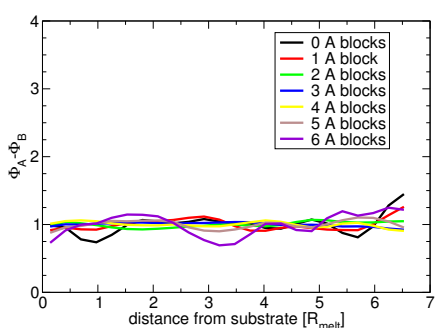

(h) $g N=0, \chi_{0} N=240$

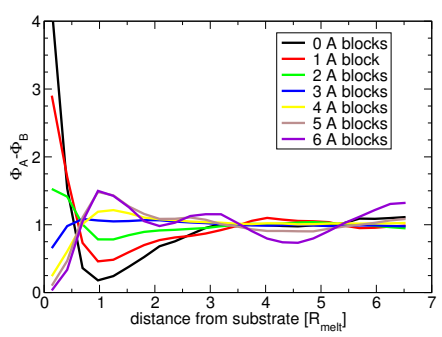

(c) $g N=1, \chi_{0} N=30$

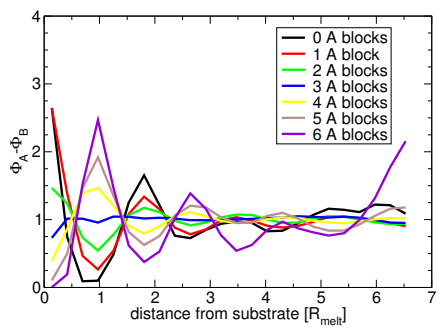

(f) $g N=1, \chi_{0} N=100$

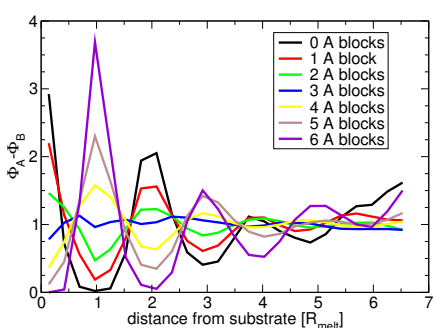

(i) $g N=1, \chi_{0} N=240$

Figure 4.7: Segregation at substrate. The segregation of different types of polymers is shown, sorted by the number of $A$ blocks the chain contains. For each group of polymers, the graph shows the number of their monomers found divided by the number of monomers expected in a homogeneous distribution. The simulation was performed - from top to bottom - for $\chi_{0} N=30$, $\chi_{0} N=100$, and $\chi_{0} N=240$. The substrate is attractive to $A$ monomers for the left figures, neutral for the middle figures, and attractive to $B$ monomers in the right figures. For $\chi_{0} N=100$ and $\chi_{0} N=240$, we see clear signals of the interphase. 


\subsubsection{Conformations at the Substrate}

When we analyzed the conformational properties of the different sequences in the bulk, see subsection 3.1.2, we found them to depend strongly on the sequence of the polymer. Resolving these two parameters, sequence and distance from the substrate, will be difficult as we can deduce from the depletion of certain sequences in specific distances from the substrate, see figure 4.7. We want to observe the change in the mean squared radius of gyration, $R_{g}^{2}$, divided into its two components parallel and perpendicular to the substrate. We have described the theory for a non-interacting substrate in subsection 4.1.1. It is uncomplicated to do the comparison for Silberberg's approach in a SCMF simulation. To this end, we take a bulk system of homopolymers, as used in the last chapter, and mirror all conformation in a virtual wall in the middle of the bulk. This changes the perpendicular part of the mean squared end-to-end distance. For comparison, we look at a homopolymer melt, confined by two reflecting walls, to determine the influence of the wall in our case, see figure 4.8(a). For a homopolymer melt, the change of conformations at a substrate has been investigated in simulations [KVY88, MBB01] and experiment [ [JPT $\left.{ }^{+} 00\right]$, which all observed a flattening of the chains at the substrate. The experiments focused on the parallel part of the radius of gyration as a function of the distance from the substrate. The profile of this quantity in our simulation with a neutral wall in figure 4.8(b) is similar to the ones in the literature.

From now on, the binning in the direction perpendicular to the substrate is performed according to the positions of the polymers' centers of mass.

The radius of gyration of a polymer is defined as

$$
R_{g}^{2}=\frac{1}{N} \sum_{i=1}^{N}\left(\vec{r}(i)-\vec{r}_{\text {com }}\right)^{2}
$$

where $\vec{r}_{\text {com }}$ is the center of mass of the polymer. When we take the x-direction as the non-periodic dimension of our simulation,

$$
R_{g, \perp}^{2}=\frac{1}{N} \sum_{i=1}^{N}\left(\vec{r}(i)_{x}-\vec{r}_{c o m, x}\right)^{2}
$$

and

$$
R_{g, \|}^{2}=R_{g}^{2}-R_{g, \perp}^{2} .
$$

For the disordered system, $\chi_{0} N=30$, we observe a flattening of the polymer conformations at the substrate, followed by them being extended perpendicular to the 


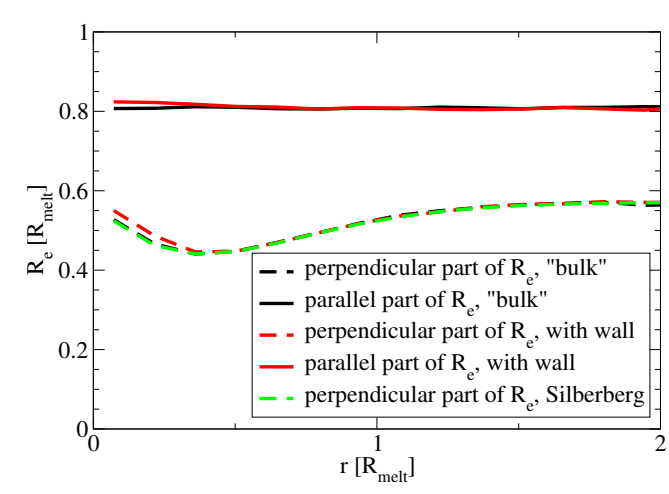

(a)

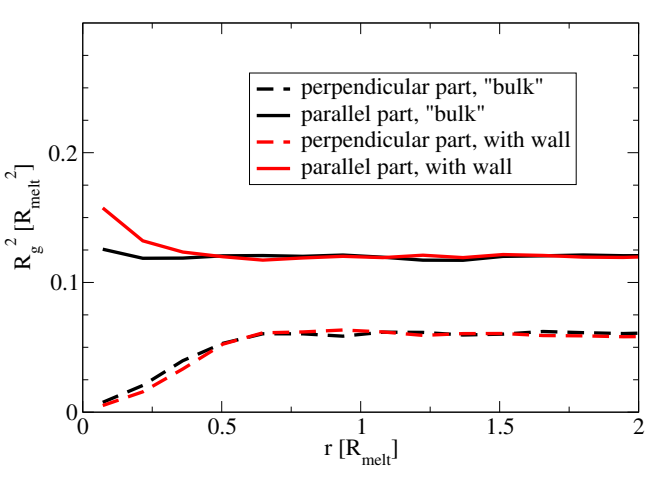

(b)

Figure 4.8: Profile of $R_{e}$ : Silberberg's argument. In figure (a) we compare the two parts of the root of the mean squared end-to-end radius, $R_{e}=\sqrt{\left\langle R_{e}^{2}\right\rangle}$, binned according to the chains' ends. We use three methods: the calculation according to Silberberg from subsection 4.1.1 ("Silberberg"), the SCMF simulation of a homopolymer melt in the bulk ("bulk"), where the chain conformations are mirrored in a virtual wall in the middle of the simulation box, and the SCMF simulation of a homopolymer melt confined by two reflective walls. The simulation in the bulk and Silberberg's argument for the perpendicular part of $R_{e}$ agree with each other, while a simple reflective wall causes deviations close to the wall. This is also seen in the parallel part of $R_{e}$, where the value for the simulations with a wall deviate from the bulk value close to it. In figure (b) we look at the mean square of the radius of gyration, binned according to the position of the chains' centers of mass. In this case, we find a much stronger deviation of the perpendicular part at the reflective wall when compared to the "bulk" value. There is also a much smaller number of chains found in the bin right at the wall.

substrate at $R_{\text {melt }}=0.5$. The peak is not strong, the profiles for $g N= \pm 1$ in figures 4.9(a) and 4.9(c) strongly resemble the melt in contact with a neutral substrate in figure 4.9(b), The conformations appear to be not as sensitive to the influence of the substrate as the compositional properties.

For the microemulsion-like structure of $\chi_{0} N=100$, the interphase is apparent in the profile of the radius of gyration as well, see figures 4.9(d) and 4.9(f). While the 
parallel component of $R_{g}^{2}$ shows that the extension of the polymers in parallel direction is smaller than the bulk value close to the substrate, the perpendicular components show peaks which mean that the polymers in this region of the peak are stretched in perpendicular direction. Thus, the formation of layers in the composition profile from figures 4.5(c) and 4.5(d) becomes visible in the conformation profile, too.

At the highest incompatibility, $\chi_{0} N=240$, we see an even stronger signal of the interphase in the perpendicular component of $R_{g}^{2}$ in figures $4.9(\mathrm{~g})$ and $4.9(\mathrm{~g})$, as well as in the parallel component. The stretching of the polymers across the layers in the interphase can be seen extending more than $3 R_{\text {melt }}$ into the simulation volume. The asymmetry between the two monomers, $\alpha$, has a large effect on this profile. For the $B$ attractive substrates, see figure 4.9(i), the second peak of the perpendicular component of $R_{g}^{2}$ is more pronounced than for the $A$-attractive substrate in figure $4.9(\mathrm{~g})$, 


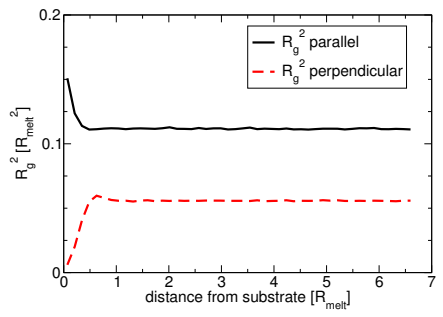

(a) $g N=-1, \chi_{0} N=30$

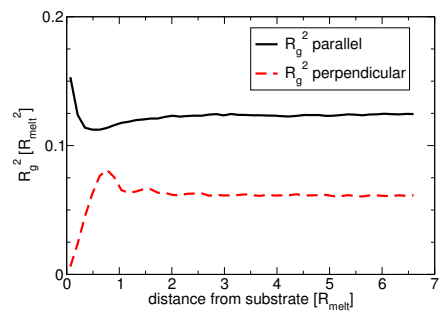

(d) $g N=-1, \chi_{0} N=100$

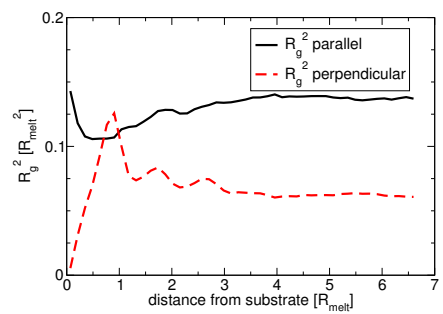

(g) $g N=-1, \chi_{0} N=240$

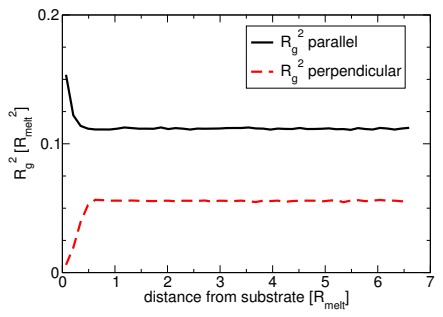

(b) $g N=0, \chi_{0} N=30$

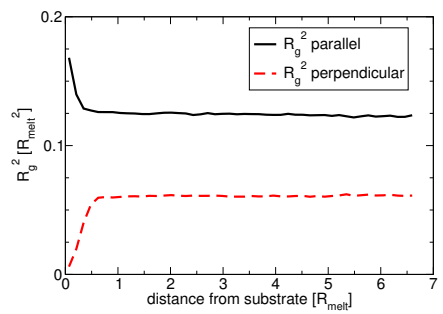

(e) $g N=0, \chi_{0} N=100$

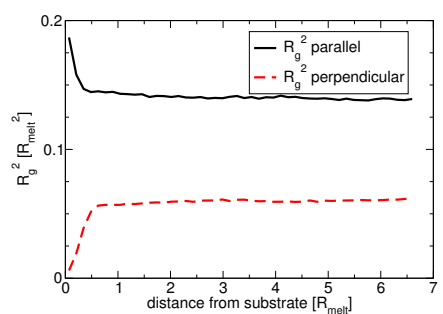

(h) $g N=0, \chi_{0} N=240$

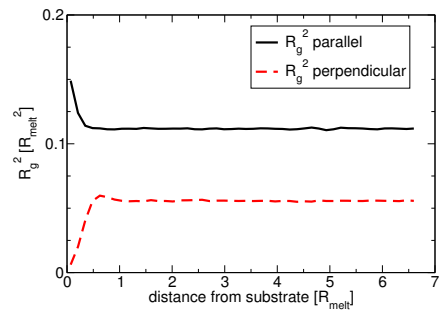

(c) $g N=1, \chi_{0} N=30$

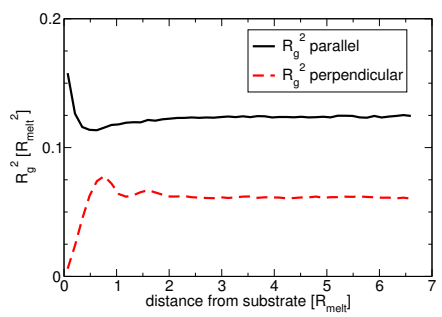

(f) $g N=1, \chi_{0} N=100$

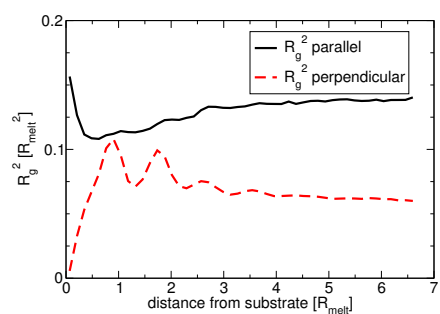

(i) $g N=1, \chi_{0} N=240$

Figure 4.9: Conformations at substrate. The parallel and perpendicular components of the squared radius of gyration are plotted as a function of the distance from the substrate. The interaction strength - from top to bottom - is $\chi_{0} N=30, \chi_{0} N=100$, and $\chi_{0} N=240$. The systems on the left have substrates which attract $A$ monomers, the center ones are confined by neutral substrates, and the systems on the left have substrates which attract $B$ monomers. We see the deformation of polymers in the interphase clearly in the case of attractive substrates and $\chi_{0} N=100,240$. 


\subsubsection{Dynamics at the Substrate}

For the dynamics at the substrate, we investigate the mean squared displacement of the centers of mass. Due to the system's anisotropy, we divide the MSD into the two components parallel and perpendicular to the substrate. Here, we differentiate the region close to the substrate into two parts which we designate as interphase and interface.

We define the perpendicular and parallel parts similarly to the definition of the radius of the gyration in the last subsection. The total MSD is defined as

$$
g_{3}(t)=\left\langle(\vec{r}(t)-\vec{r}(0))^{2}\right\rangle
$$

which we can divide again into the perpendicular

$$
g_{3, \perp}(t)=\left\langle\left(\vec{r}_{x}(t)-\vec{r}_{x}(0)\right)^{2}\right\rangle
$$

and parallel

$$
g_{3, \|}(t)=g_{3}(t)-g_{3, \perp}(t)
$$

parts.

For the definition of interface and interphase, we adhere to the composition profiles in figure 4.5. With the help of these profiles, we take the interface as extending $0.5 R_{\text {melt }}$ into the simulation volume, and the interphase from $0.5 R_{\text {melt }}$ to $2.4 R_{\text {melt }}$.

For the low incompatibility, $\chi_{0} N=30$, we notice a slowing-down of the dynamics perpendicular to the substrate in the interface, when comparing the neutral substrate in figure 4.10(b) with the selectively attractive substrates in figures 4.10(a) and 4.10(c). Since the substrate is attractive to one type of monomers, polymers containing this type prefer to stay in the interface region. The motion parallel to the substrate is neither slowed down nor enhanced when comparing interface with interphase.

With a higher incompatibility, $\chi_{0} N=100$, two new properties of the MSD turn up which are interrelated. A speed-up parallel to the substrate and a slowing-down of the perpendicular motion appear when we compare the motion of the polymer melt in contact with a neutral substrate in figure $4.10(\mathrm{e})$ to the motion with a selectively attractive substrate, see figures $4.10(\mathrm{~d})$ and $4.10(\mathrm{f})$. The formation of layers allows the chains to move faster parallel to the substrate in the interface than in the interphase, since they do not encounter domain boundaries which would slow them down. Perpendicular to the substrate, it is the other way around. The domain boundaries, which are very 


\section{Selectively Attractive Substrate}

pronounced in this direction, hinder this type of motion. This layering is visible in the concentration profile, see figures $4.5(\mathrm{c})$ and $4.5(\mathrm{~d})$.

When the incompatibility reaches $\chi_{0} N=240$, the higher value of the asymmetry parameter $\alpha=0.05$ influences the dynamics as well. At this point, the dynamics differ between the $A$-attractive substrate in figure $4.10(\mathrm{~g})$ and the $B$-attractive substrate in figure 4.10(i), First let us consider the similarities. The layering which extends further into the interphase, results in a slowing down of the perpendicular motion, when compared to the neutral substrate in figure 4.10(h), and an enhanced motion parallel to the substrate. However, while the perpendicular motion in the interface practically ceases for the $A$-attractive substrate, an escape of the interface is still possible for polymers when in contact with a $B$-attractive substrate. There is also less of a difference in the motion parallel to the substrate in the interphase for the $B$-attractive substrate, while the motion parallel to the substrate in the interface is still greater than in the interphase for the $A$-attractive substrate. The composition profiles in figures 4.5(e) and 4.5(f), and the segregation of sequences in figures 4.7(g) and 4.7(i) would not suggest such a great difference, while the $R_{g}^{2}$-profiles in figures 4.9(g) and 4.9(i) also show qualitatively different behaviour between the $A$ - and $B$-attractive substrates.

These results are summed up in table 4.1, where the apparent diffusivity is given, normalized by the bulk diffusivity.

The extreme slowing-down of the dynamics perpendicular to the substrate means that the diffusion of the polymers is hindered strongly once the compositional structure has been formed. This is in agreement with experimental findings by Diethert et al. [DPWMB10], who found the statistical copolymer melts in contact with a substrate, changed their composition profile very slowly after the solvent had evaporated. 

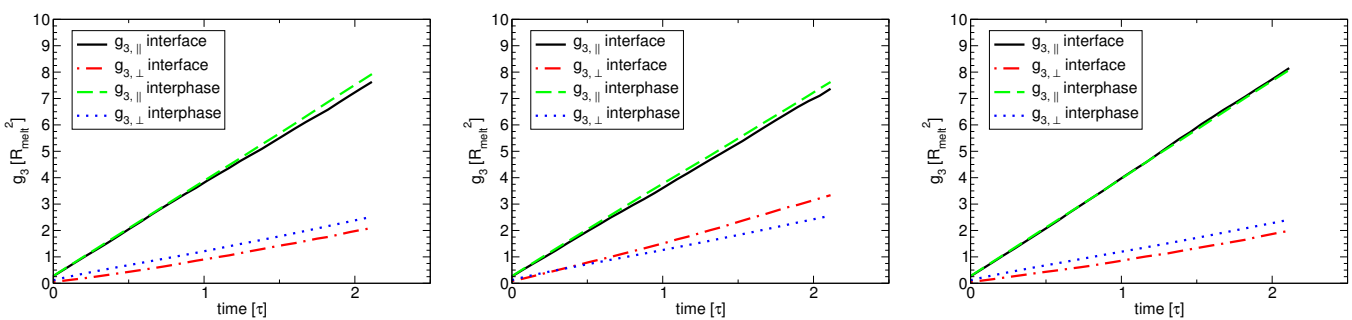

(a) $g N=-1, \chi_{0} N=30$

(b) $g N=0, \chi_{0} N=30$

(c) $g N=1, \chi_{0} N=30$
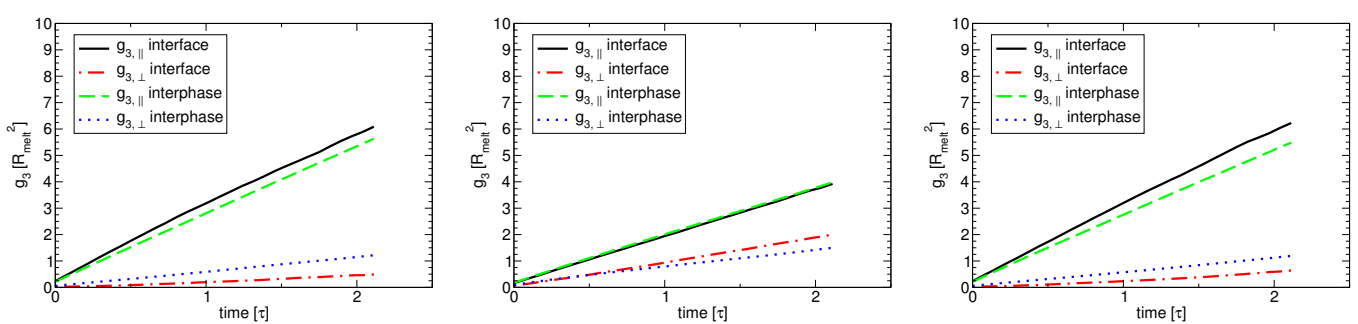

(d) $g N=-1, \chi_{0} N=100$

(e) $g N=0, \chi_{0} N=100$

(f) $g N=1, \chi_{0} N=100$
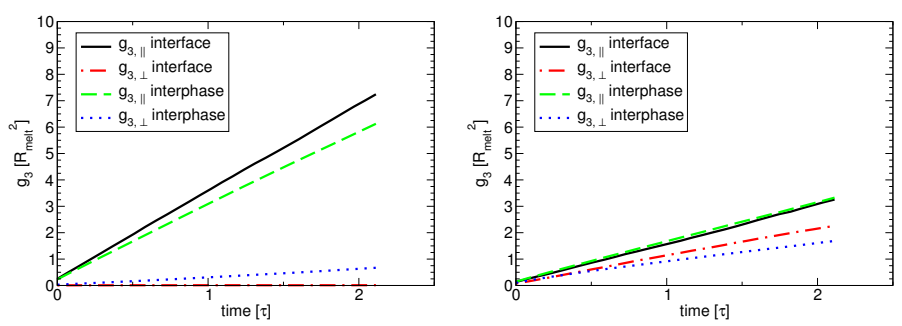

(g) $g N=-1, \chi_{0} N=240$

(h) $g N=0, \chi_{0} N=240$

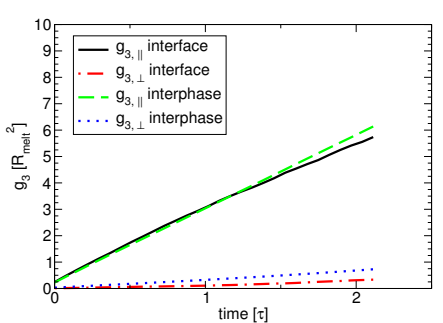

(i) $g N=1, \chi_{0} N=240$

Figure 4.10: MSD at substrate. The parallel and perpendicular components of the mean squared displacement are plotted for the interface and interphase. The interaction strength - from top to bottom - is $\chi_{0} N=30, \chi_{0} N=100$, and $\chi_{0} N=240$. The systems on the left have substrates which attract $A$ monomers, the center ones are confined by neutral substrates, and the systems on the left have substrates which attract $B$ monomers. When an interphase forms - figures (d), (f), (g), and (i) - there is a speed-up of the parallel dynamics in interface and interphase, combined with a slowingdown of the perpendicular dynamics. 


\begin{tabular}{|c|r|l|l|l|l|}
\hline \multirow{2}{*}{$\chi_{0} N$} & \multirow{2}{*}{$g N$} & \multicolumn{3}{|c|}{ Interface } & \multicolumn{2}{c|}{ Interphase } \\
\cline { 2 - 6 } & & parallel & perpendicular & parallel & perpendicular \\
\hline \multirow{3}{*}{30} & -1 & 1.0 & 0.6 & 1.0 & 0.6 \\
\cline { 2 - 6 } & 0 & 1.0 & 0.9 & 1.0 & 0.6 \\
\cline { 2 - 6 } & 1 & 1.1 & 0.5 & 1.1 & 0.6 \\
\hline \multirow{4}{*}{100} & -1 & 1.4 & 0.2 & 1.3 & 0.6 \\
\cline { 2 - 6 } & 0 & 0.9 & 0.9 & 0.9 & 0.7 \\
\cline { 2 - 6 } & 1 & 1.5 & 0.3 & 1.3 & 0.5 \\
\hline \multirow{3}{*}{240} & -1 & 1.8 & 0.002 & 1.5 & 0.3 \\
\cline { 2 - 6 } & 0 & 0.8 & 1.1 & 0.8 & 0.8 \\
\cline { 2 - 6 } & 1 & 1.4 & 0.2 & 1.5 & 0.3 \\
\hline
\end{tabular}

Table 4.1: Apparent diffusivity at a selective substrate. The apparent diffusivity is calculated by dividing the parallel component of the measured diffusivity by the two thirds of the diffusivity of the bulk random block copolymer melt at the same incompatibility $\chi_{0} N$, and the perpendicular component by one third of this diffusivity. 


\subsection{Mapping with a Selective Substrate}

For the mapping procedure from the soft, coarse-grained model to the Lennard-Jones bead-spring model, we follow the same procedure as in subsection 3.1.5. We decrease the chain discretization from $N=120$ to $N=60$ by taking the center of mass of two neighboring monomers of the soft, coarse-grained model as the monomer in the Lennard-Jones bead-spring model. The transformation of the coordinates is slightly different. Since the potential in the Lennard-Jones bead-spring model has a repulsive component, we map the " $\mathrm{x}$ "-coordinate by compressing the melt such that no monomer can be found within $1 \sigma$ of the substrate in the starting configurations of the LennardJones bead-spring model. Then we proceed with $N V E$ simulations with a limited movement per time step for 100 time steps. Following this is a simulation in the $N V T$ ensemble for $10^{6}$ time steps, and then a simulation in tensionless $N P_{t} T$ ensemble for up to $10^{7}$ time steps. We look at the concentration profile over this time frame.

Both the disordered system with $\varepsilon_{A A}=1.1 / \chi_{0} N=30$, see figure 4.11, and the highly segregated one with $\varepsilon_{A A}=1.6 / \chi_{0} N=240$, see figure 4.13 , reach a stable state within $0.12 \tau$. For the intermediate system with $\varepsilon_{A A}=1.3 / \chi_{0} N=100$, see figure 4.12, the time frame we look at appears to be insufficient to achieve or return to the long-range order of the interphase we observe in various quantities in this chapter.

The regions close to the substrates are the most interesting regions for the question of the equilibration after mapping, spanning from 0 to $4 R_{\text {melt }}$ and 9.6 to 13.6 $R_{\text {melt }}$. While there are fluctuations in the composition of the "bulk" region between the interphases, even after the equilibration of the system, the structure formed in the interphase, for values of $\varepsilon_{A A}$ that lead to microemulsion-like structures in the bulk, does not change on the observed time scale. Thus, the strong change in the right-hand interphase composition after mapping for a $B$-attractive substrate at $\varepsilon_{A A}=1.3$, see figure 4.12(b), means that we encounter a problem in this case. The equilibration after mapping for an $A$-attractive substrate and $\varepsilon_{A A}=1.3$, see the right interphase of figure 4.12(a), also shows a similar, but not quite as drastic behaviour.

At the same time, the lack of structure in the case of the disordered melt, $\varepsilon_{A A}=1.1$, and the highly ordered structure for the high incompatibility, $\varepsilon_{A A}=1.6$, facilitate the equilibration. 


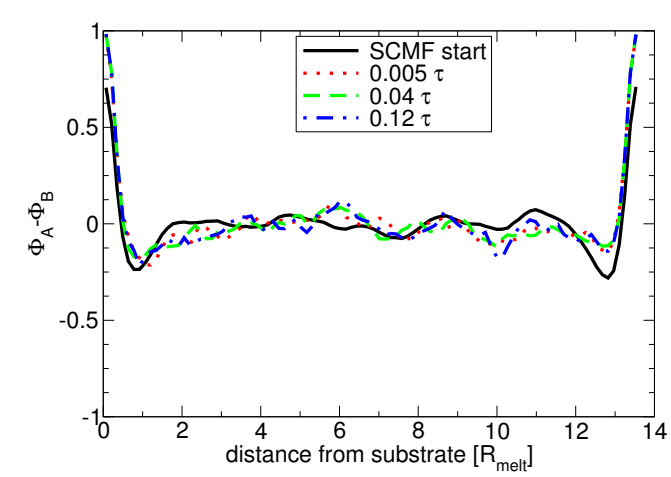

(a) $A$ monomers

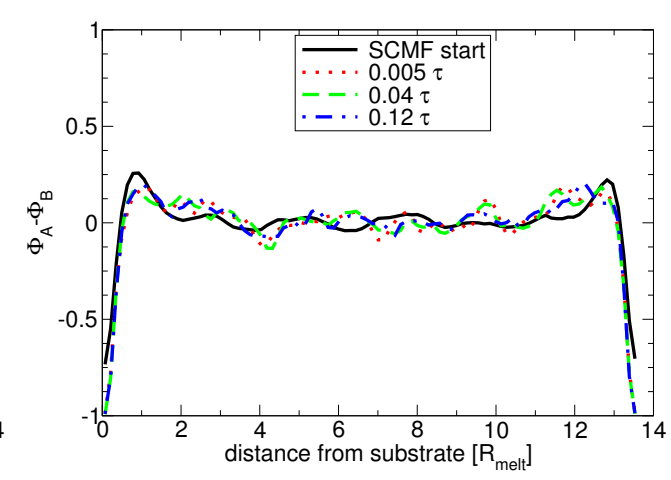

(b) $B$ monomers

Figure 4.11: Time evolution, mapping at substrate: $\varepsilon_{A A}=1.1$. We observe the time evolution when mapping from the soft, coarse-grained model with $\chi_{0} N=$ 30 to the Lennard-Jones bead-spring model with $\varepsilon_{A A}=1.1$. The left figure shows substrates attracting $A$-monomers, while the right figure is for substrates attracting $B$-monomers. In this parameter set we are still in the disordered phase so the system equilibrates quickly.

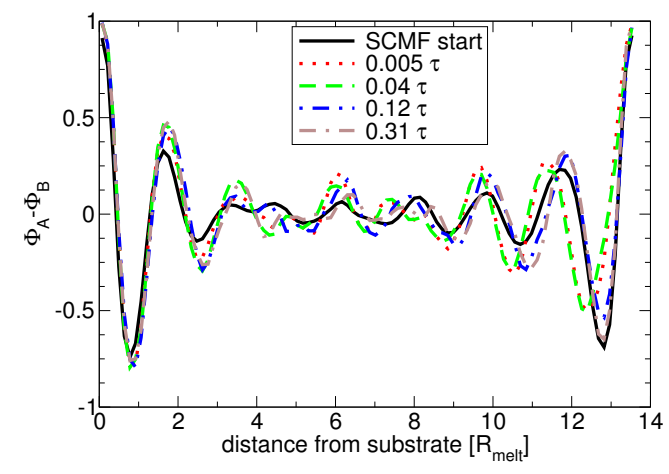

(a) $A$ monomers

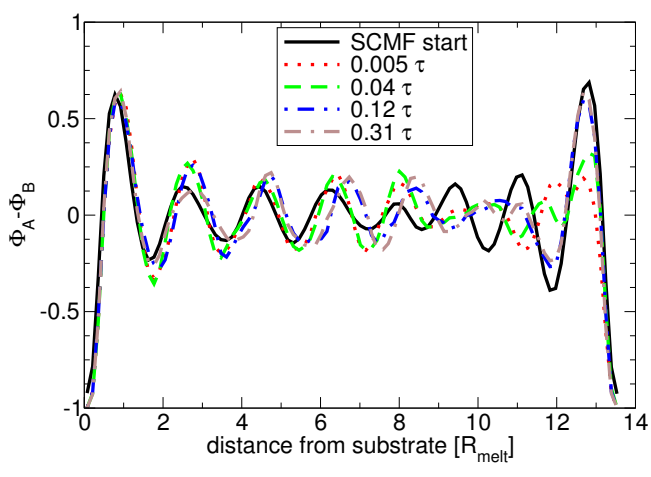

(b) $B$ monomers

Figure 4.12: Time evolution, mapping at substrate: $\varepsilon_{A A}=1.3$. We observe the time evolution when mapping from the soft, coarse-grained model with $\chi_{0} N=$ 100 to the Lennard-Jones bead-spring model with $\varepsilon_{A A}=1.3$. The left figure shows substrates attracting $A$-monomers, while the right figure is for substrates attracting $B$-monomers. We see in both figures that there is an intermediate change in the composition before the profile becomes stable. 


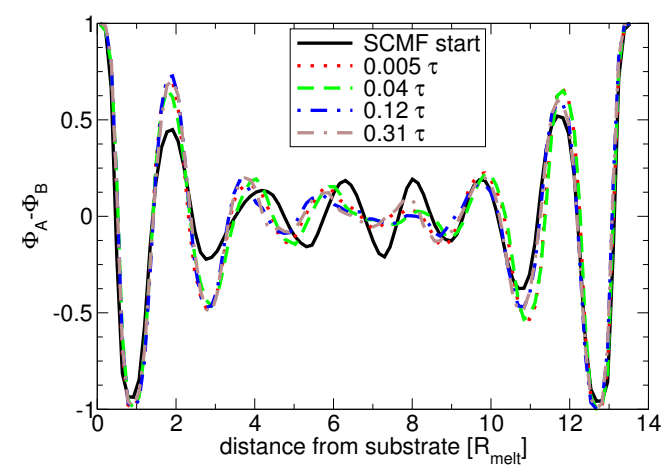

(a) $A$ monomers

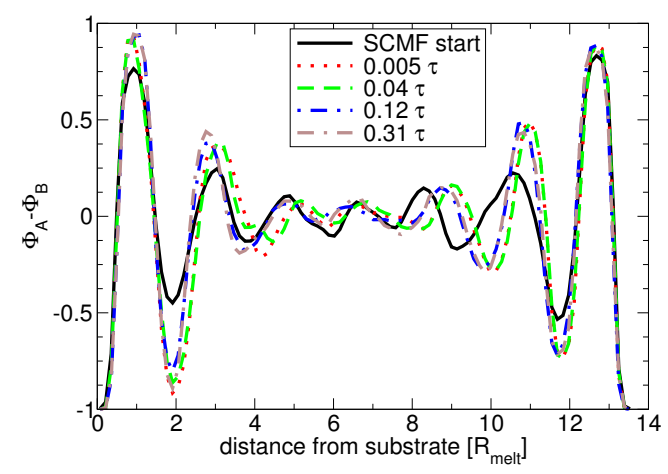

(b) $B$ monomers

Figure 4.13: Time evolution, mapping at substrate: $\varepsilon_{A A}=1.6$. We observe the time evolution when mapping from the soft, coarse-grained model with $\chi_{0} N=240$ to the Lennard-Jones bead-spring model with $\varepsilon_{A A}=1.6$. The left figure shows substrates attracting $A$-monomers, while the right figure is for substrates attracting $B$-monomers. The strong interaction and the distinct layering at the outset facilitate a fast equilibration of the system. 


\subsection{Mechanical Properties}

When comparing mechanical properties of the bulk random block copolymer melt in section 3.2, we unsuccessfully tried to correlate the shear modulus calculated from NEMD simulations with the shear modulus from the local elastic modulus tensor. Now we have a second opportunity to look at the local mechanical properties, this time depending on the distance from the substrate. We take the MD systems as prepared in 4.2.2 and quench them to $k_{B} T=0.3$. Then we let them age in the $N P_{t} T$ ensemble with $P_{t}=0$ for $4 \cdot 10^{6}$ time steps, and $3 \cdot 10^{6}$ time steps after switching to $N V T$.

For the measurement of the elastic properties as in equation 3.29, we take an average over $6 \cdot 10^{6}$ time steps every 200 time steps. One time step was found in section 3.1 to be equivalent to $4.8 \cdot 10^{-8} \tau$.

For the measurement of the shear modulus in NEMD simulations, we deform our system. With the " $x$ "-direction as the non-periodic direction, we shear the system by changing $\epsilon_{4}$. The strain is increased by $\Delta \epsilon_{4}=5 \cdot 10^{-4}$ every $10^{4}$ time steps and we calculate the stress for each of these intervals as an average with a step size of 200 time steps. In the linear regime up to a strain of $\epsilon_{4}=10^{-2}$, we calculate the shear modulus locally as described in subsection 3.2.3.

The calculations for both methods are performed in slabs parallel to the substrate. The width of each slab is $3.4 \sigma$, therefore we only have to define the subdivision of the volume differently than in the bulk case.

The results of these simulations are shown for $\varepsilon_{A A}=1.1$ in figure 4.14. In the vicinity of the substrate, both methods show similar results. They show the lower shear modulus at the substrate for a $B$-attractive substrate, see figure 4.14(b), In the case of an $A$-attractive substrate, see 4.14(a), there are two effects in the interface region which counteract each other. While the shear modulus in regions of high concentration of $A$-monomers is slightly higher, the density at the substrate is lower than in the bulk. Thus, the shear modulus in the interfacial region in this case is greater than for the $B$-attractive substrate but still differs from the bulk value.

For $\varepsilon_{A A}=1.3$, the results of the calculations of the shear modulus are presented in figure 4.15. In the vicinity of the substrate, the two methods agree well. The structure at the interface and in the interphase is represented by a lower shear modulus at the substrate for a $B$-attractive substrate, see figure $4.15(\mathrm{~b})$, followed by a higher shear modulus due to the higher concentration of $A$-monomers in the next layer. In the 
case of the $A$-attractive substrate, see 4.15(a), the interplay between lower density and higher shear modulus of the $A$-rich domains results in a value of the shear modulus in the interfacial region which is close to the bulk value.

The strongest interaction of $\varepsilon_{A A}=1.6$ leads to the best agreement of the two different measurements. The profiles of the shear modulus in both methods and for both types of substrate agree very well up to a distance of $2.5 R_{\text {melt }}$ from the substrate, see figure 4.16. The magnitude of the shear modulus here varies the strongest in the three simulations.

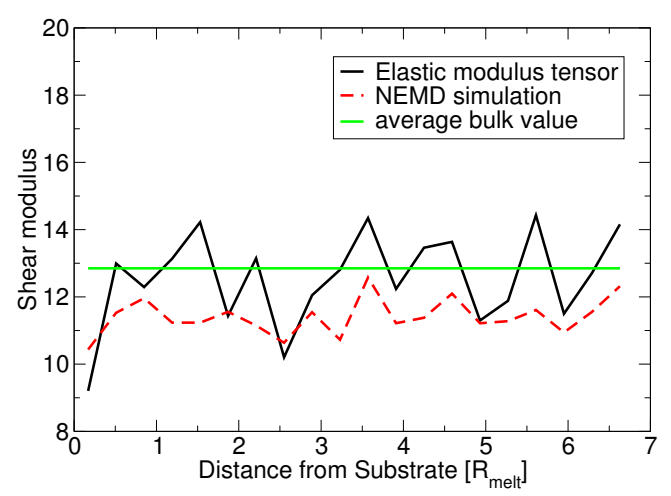

(a) $A$-attractive

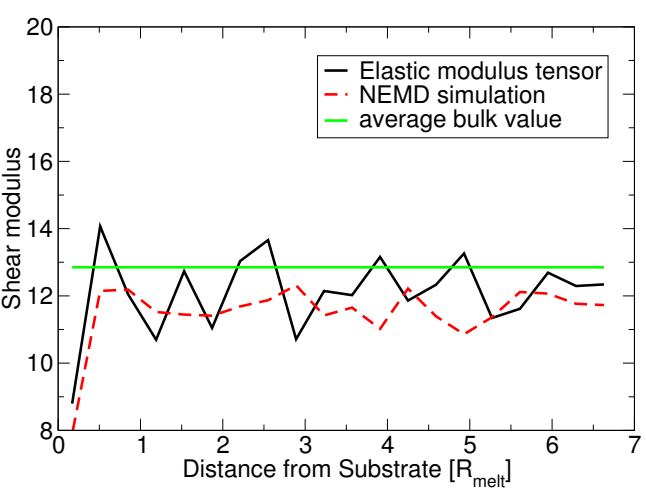

(b) $B$-attractive

Figure 4.14: Shear modulus profile: $\varepsilon_{A A}=1$.1. In these two figures we compare the results for the shear modulus, as calculated from the elastic modulus tensor and NEMD simulations for a random block copolymer melt in contact with a selectively attractive substrate. The simulations were performed at $k_{B} T=0.3, \varepsilon_{A A}=1.1$ and $\varepsilon_{\text {wall }}=2.0$. There is a drop of the shear modulus in both cases at the substrate, due to the lower density in this region. 


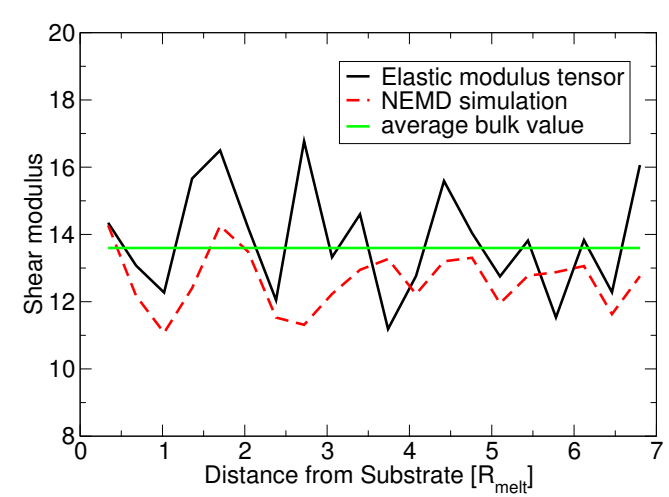

(a) A-attractive

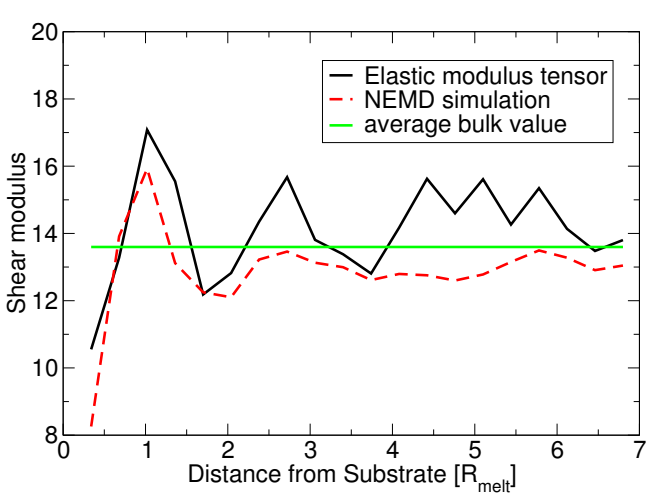

(b) $B$-attractive

Figure 4.15: Shear modulus profile: $\varepsilon_{A A}=1$.3. In these two figures we compare the results for the shear modulus, as calculated from the elastic modulus tensor and NEMD simulations for a random block copolymer melt in contact with a selectively attractive substrate. The simulations were performed at $k_{B} T=0.3, \varepsilon_{A A}=1.3$ and $\varepsilon_{\text {wall }}=2.0$. We see a signal of the interface region, as well as a hint of the interphase in the shear modulus profiles.

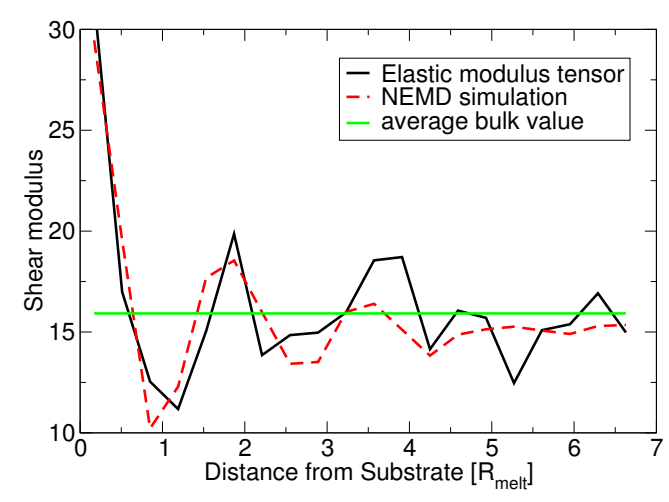

(a) A-attractive

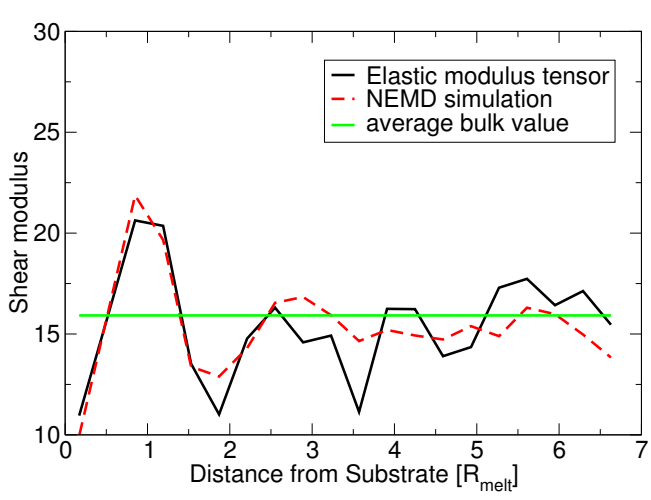

(b) $B$-attractive

Figure 4.16: Shear modulus profile: $\varepsilon_{A A}=1$.6. In these two figures we compare the results for the shear modulus, as calculated from the elastic modulus tensor and NEMD simulations for a random block copolymer melt in contact with a selectively attractive substrate. The simulations were performed at $k_{B} T=0.3, \varepsilon_{A A}=1.6$ and $\varepsilon_{\text {wall }}=3.0$. The layers of the interphase show up clearly in the profiles of the shear modulus. 


\title{
5 Conclusion and Outlook
}

\author{
"They wanted facts. Facts! \\ They demanded facts from him, \\ as if facts could explain anything." \\ (Joseph Conrad)
}

In this thesis, I have used computer simulations to investigate the single-chain properties, morphology, dynamics, and local mechanical properties of random block copolymer melts in the volume and in contact with a selective substrate.

In chapter 2 we have described the two distinctly different models and methods we have used to simulate random block copolymer melts. A soft, coarse-grained model in conjunction with SCMF simulations was used because of its computational efficiency, thus being able to equilibrate a system fast and explore the role of model parameters systematically, while the Lennard-Jones bead-spring model has slower, more accurate dynamics, and allows us to probe elastic properties due to the harsh repulsion of the monomers.

In chapter 3, we have investigated the bulk properties of random block copolymer melts with the help of the two aforementioned model/method combinations. We have found parameters for the two models which result in similar mesoscopic structure of and similar conformations in the random block copolymer melt. With increasing incompatibility of the two monomer types, both models form microemulsion-like structures. For a high value of the incompatibility in long-running SCMF simulations, we have found an indication of a lamellar phase, which is in accordance with mean-field calculations [FM91, FML92] and recent simulations [GKKC11]. The growing length scale of the domains with growing incompatibility is, however, in contradiction to the mean-field predictions [FM91, FML92]. The similarity of the mesoscopic structures of the two models can be used to significantly reduce the time needed for an equilibration in MD simulations. When using equilibrated SCMF configurations as starting configurations for MD simulations, the equilibration time is reduced by an order of 


\section{Conclusion and Outlook}

magnitude at the least. A comparison of the dynamic properties of the two models, the mean squared displacement and the end-to-end vector autocorrelation function, shows differences between the two models for pairs of parameters at which the structural properties agree. The slowing-down of the dynamics in the Lennard-Jones beadspring model is more pronounced than for the soft, coarse-grained model with rising incompatibility. We have introduced a method to calculate the global and local elastic properties based on the calculation of the local elastic modulus tensor [Lut88, Lut89]. When comparing experimental results to the global elastic properties of the LennardJones bead-spring model, the elastic properties from our simulations are three orders of magnitude too small. However, the relation between shear and bulk modulus is similar to the one observed in poly(methyl methacrylate) glasses. We have also investigated the local elastic properties of random block copolymer melts for different degrees of incompatibility. We see a clear difference of the elastic properties between $A$-rich, $B$-rich domains and their interfaces when the structure of the random block copolymer melt is microemulsion-like. However, we are not able to reproduce a correlation between the local shear stress and the local shear modulus in a sheared system, which had previously been found [ $\left.\mathrm{YJvW}^{+} 04\right]$.

The mapping between the two models, and the way to access elastic properties in the Lennard-Jones bead-spring model, provide a great opportunity to investigate the elastic properties of random block copolymer melts in even more detail in the future, for example under larger deformations.

Finding and describing properties of the interphase of a random block copolymer melt in contact with a selectively attractive substrate was the main aim of chapter 4. The linear response theory as used for diblock copolymer melts [MM96] has proven to be very effective in predicting the composition profile of the melt in contact with a selective substrate from the static structure factor of the composition of the bulk melt. The layer-like structure, which forms for incompatibilities that show microemulsionlike structures in the bulk, gives rise to a region called the interphase [BB95]. The interphase is even more pronounced when regarding the concentration of the different polymer species found in a random block copolymer melt, categorized according to the number of $B$-blocks they contain. Looking at the conformational profile of the interphase, we have found an indication of the interphase in observables like the radius of gyration which is perpendicular to the plane of the substrate. Especially for higher incompatibility of the monomers, the layering of the composition clearly influences 
the conformations of the polymers. Furthermore, we observe a speed-up of the motion parallel to the substrate in the interface and interphase regions, while the motion perpendicular to the substrate in these two regions is greatly reduced. The mapping from the soft, coarse-grained model to the Lennard-Jones bead-spring model in the case of a selective substrate is less straightforward than in the bulk. The low and high values of incompatibility show a speed-up of equilibration time, whereas the intermediate value, where a microemulsion-like structure with broad interfaces between domains forms in the bulk, takes longer to equilibrate due to strong fluctuations in the interphase. Finally, the local shear modulus in the interface and interphase deviates from the bulk values as well. The profile of the shear modulus, especially in the interface region, is the result of an intricate interplay of concentration of monomer types and the local density in this region.

The fractionation of chain types, dynamical and conformational changes in the interphase, as well as a change in elastic properties, give a very good starting point for the further investigation and characterization of this small but important region of polymers in contact with a selective solid substrate. The proportion of the interphase with respect to the whole volume can be enhanced by using nanoparticles, where each nanoparticle generates its own interphase. As we have seen from the analysis of the dynamics, it change significantly in the interface and interphase. We have worked with fixed sequences, but how would the dynamics change and be influenced by an (ir)reversible polymerization process? The influence of the coarse-grained model's coarseness on the interface [MSD $\left.{ }^{+} 11\right]$ or interphase properties is a topic which merits investigation for random block copolymers as well. 



\section{Bibliography}

$\left[\mathrm{AEG}^{+}\right.$03] R. Auhl, R. Everaers, G. S. Grest, K. Kremer, and S. J. Plimpton. Equilibration of long chain polymer melts in computer simulations. The Journal of Chemical Physics, 119(24):12718-12728, 2003.

[AM76] N. W. Ashcroft and N. D. Mermin. Solid State Physics. Harcourt, Orlando, 1976.

[AT87] M. P. Allen and D. J. Tildesley. Computer Simulation of Liquids. Clarendon Press, Oxford, 1987.

[BB95] J. Baschnagel and K. Binder. On the influence of hard walls on structural properties in polymer glass simulation. Macromolecules, 28(20):6808-6818, 1995.

$\left[\mathrm{BBD}^{+} 00\right]$ J. Baschnagel, K. Binder, P. Doruker, A. A. Gusev, O. Hahn, K. Kremer, W. L. Mattice, F. Müller-Plathe, M. Murat, W. Paul, S. Santos, U. W. Suter, and V. Tries. Bridging the gap between atomistic and coarsegrained models of polymers: Status and perspectives. In Advances in Polymer Science: Viscoelasticity, Atomistic Models, Statistical Chemistry, volume 152, pages 41-156. Springer-Verlag Berlin, 2000.

[BF99] F. S. Bates and G. H. Fredrickson. Block copolymers - designer soft materials. Physics Today, 52(2):32-38, 1999.

$\left[\mathrm{BHM}^{+}\right.$08] S. Bhattacharya, H.-P. Hsu, A. Milchev., V. G. Rostiashvili, and T. A. Vilgis. Adsorption of multiblock and random copolymer on a solid surface: Critical behavior and phase diagram. Macromolecules, 41(8):2920-2930, 2008. 


\section{Bibliography}

[BHMW11] B. Bae, T. Hoshi, K. Miyatake, and M. Watanabe. Sulfonated block poly(arylene ether sulfone) membranes for fuel cell applications via oligomeric sulfonation. Macromolecules, 44(10):3884-3892, 2011.

[Bin95] K. Binder, editor. Monte Carlo and Molecular Dynamics Simulations in Polymer Science. Oxford University Press, New York, 1995.

[BPBB99] C. Bennemann, W. Paul, J. Baschnagel, and K. Binder. Investigating the influence of different thermodynamic paths on the structural relaxation in a glass-forming polymer melt. Journal of Physics: Condensed Matter, 11(10):2179, 1999.

[BPBBD98] C. Bennemann, W. Paul, K. Binder, and Burkhard B. Dünweg. Molecular-dynamics simulations of the thermal glass transition in polymer melts: $\alpha$-relaxation behavior. Physical Review E, 57:843-851, 1998.

[CK88] I. Carmesin and K. Kremer. The bond fluctuation method: a new effective algorithm for the dynamics of polymers in all spatial dimensions. Macromolecules, 21(9):2819-2823, 1988.

[DB91] H. P. Deutsch and K. Binder. Interdiffusion and self-diffusion in polymer mixtures: A monte carlo study. The Journal of Chemical Physics, 94(3):2294-2304, 1991.

[DGP ${ }^{+}$99] C. Donati, S. C. Glotzer, P. H. Poole, W. Kob, and S. J. Plimpton. Spatial correlations of mobility and immobility in a glass-forming lennardjones liquid. Physical Review E, 60:3107-3119, 1999.

[DM06] K. C. Daoulas and M. Müller. Single chain in mean field simulations: Quasi-instantaneous field approximation and quantitative comparison with monte carlo simulations. The Journal of Chemical Physics, 125(18):184904, 2006.

[DMdP $\left.{ }^{+} 06\right]$ K. C. Daoulas, M. Müller, J. J. de Pablo, P. F. Nealey, and G. D. Smith. Morphology of multi-component polymer systems: single chain in mean field simulation studies. Soft Matter, 2:573-583, 2006. 
$\left[\mathrm{DMS}^{+}\right.$06] K. C. Daoulas, M. Müller, M. P. Stoykovich, S.-M. Park, Y. J. Papakonstantopoulos, J. J. de Pablo, P. F. Nealey, and Harun H. H. H. Solak. Fabrication of complex three-dimensional nanostructures from self-assembling block copolymer materials on two-dimensional chemically patterned templates with mismatched symmetry. Physical Review Letters, 96:036104, 2006.

$\left[\mathrm{DMS}^{+} 08\right]$ K. C. Daoulas, M. Müller, M. P. Stoykovich, H. Kang, J. J. de Pablo, and P. F. Nealey. Directed copolymer assembly on chemical substrate patterns: A phenomenological and single-chain-in-mean-field simulations study of the influence of roughness in the substrate pattern. Langmuir, 24(4):1284-1295, 2008.

[DPWMB10] A. Diethert, Y. Peykova, N. Willenbacher, and P. Müller-Buschbaum. Near-surface composition profiles and the adhesive properties of statistical copolymer films being model systems of pressure sensitive adhesive films. Applied Materials \& Interfaces, 2(7):2060-2068, 2010.

[DS04] Y. Ding and A. P. Sokolov. Comment on the dynamic bead size and kuhn segment length in polymers: Example of polystyrene. Journal of Polymer Science Part B: Polymer Physics, 42(18):3505-3511, 2004.

[EEV10] B. Ebeling, M. Eggers, and P. Vana. Ideal molecular weight distributions of multiblock copolymers prepared via raft polymerization. Macromolecules, 43(24):10283-10290, 2010.

[EMS ${ }^{+}$07] E. W. Edwards, M. Müller, M. P. Stoykovich, H. H. Solak, J. J. de Pablo, and P. F. Nealey. Dimensions and shapes of block copolymer domains assembled on lithographically defined chemically patterned substrates. Macromolecules, 40(1):90-96, 2007.

[Erm75] D. L. Ermak. A computer simulation of charged particles in solution. i. technique and equilibrium properties. The Journal of Chemical Physics, 62(10):4189-4196, 1975.

[Flo73] P. J. Flory. The challenge to macromolecular science. International Journal of Polymeric Materials, 2(4):265-269, 1973. 


\section{Bibliography}

[FM91] G. H. Fredrickson and S. T. Milner. Thermodynamics of random copolymer melts. Physical Review Letters, 67(7):835-838, 1991.

[FML92] G. H. Fredrickson, S. T. Milner, and L. Leibler. Multicritical phenomena and microphase ordering in random block copolymers melts. Macromolecules, 25(23):6341-6354, 1992.

[Fre87] G. H. Fredrickson. Surface ordering phenomena in block copolymer melts. Macromolecules, 20(10):2535-2542, 1987.

[FS02] D. Frenkel and B. Smit. Understanding Molecular Simulation. Academic Press, San Diego, second edition, 2002.

[GC94] L. Gutman and A. K. Chakraborty. Surface-induced ordering for confined random block copolymers. The Journal of Chemical Physics, 101(11):10074-10091, 1994.

[GK86] G. S. Grest and K. Kremer. Molecular dynamics simulation for polymers in the presence of a heat bath. Physical Review A, 33(5):36283631, 1986.

[GKKC11] A. A. Gavrilov, Y. V. Kudryavtsev, P. G. Khalatur, and A. V. Chertovich. Microphyse separation in regular and random copolymer melts by dpd simulations. Chemical Physics Letters, 503(4-6):277-282, 2011.

[GvHS ${ }^{+94]}$ D. W. Grijpma, R. D. A. van Hofslot, H. Supèr, A. J. Nijenhuis, and A. J. Pennings. Rubber toughening of poly(lactide) by blending and block copolymerization. Polymer Engineering \& Science, 34(22):1674-1684, 1994.

[HDS96] W. Humphrey, A. Dalke, and K. Schulten. VMD - Visual Molecular Dynamics. Journal of Molecular Graphics, 14:33-38, 1996.

$\left[\mathrm{HEH}^{+} 80\right]$ W. G. Hoover, D. J. Evans, R. B. Hickman, A. J. C. Ladd, W. T. Ashurst, and B. Moran. Lennard-jones triple-point bulk and shear viscosities. green-kubo theory, hamiltonian mechanics, and nonequilibrium molecular dynamics. Physical Review A, 22:1690-1697, 1980. 
[He175] E. Helfand. Theory of inhomogeneous polymers: Fundamentals of the gaussian random-walk model. The Journal of Chemical Physics, 62(3):999-1005, 1975.

$\left[\mathrm{HGK}^{+} 04\right]$ M. A. Hickner, H. Ghassemi, Y. S. Kim, B. R. Einsla, and J. E. McGrath. Alternative polymer systems for proton exchange membranes (pems). Chemical Reviews, 104(10):4587-4611, 2004.

$\left[\mathrm{HHG}^{+} 94\right]$ D. A. Hajduk, P. E. Harper, S. M. Gruner, C. C. Honeker, G. Kim, E. L. Thomas, and L. J. Fetters. The gyroid: A new equilibrium morphology in weakly segregated diblock copolymers. Macromolecules, 27(15):4063-4075, 1994.

[HK08] V. A. Harmandaris and K. Kremer. Quantitative study of polymer dynamics through hierarchical multi-scale dynamic simulations. arXiv, 0809.1518v1 [cond-mat.soft], 2008.

[HM02] J. Houdayer and M. Müller. Deviations from the mean-field predictions for the phase behaviour of random copolymers melts. Europhysics Letters, 58(5):660, 2002.

[HM04] J. Houdayer and M. Müller. Phase diagram of random copolymer melts: A computer simulation study. Macromolecules, 37(11):4283-4295, 2004.

[HM08] M. Hömberg and M. Müller. Generating multichain configurations of an inhomogeneous melt from the knowledge of single-chain properties. The Journal of Chemical Physics, 128:224911, 2008.

[HM10] M. Hömberg and M. Müller. Main phase transition in lipid bilayers: Phase coexistence and line tension in a soft, solvent-free, coarsegrained model. The Journal of Chemical Physics, 132:155104, 2010.

[HN81] K. M. Hong and J. Noolandi. Theory of inhomogeneous multicomponent polymer systems. Macromolecules, 14(3):727-736, 1981.

[Hoo85] W. G. Hoover. Canonical dynamics: Equilibrium phase-space distributions. Physical Review A, 31(3):1695-1697, 1985. 


\section{Bibliography}

[Hoo86] W. G. Hoover. Constant-pressure equations of motion. Physical Review A, 34(3):2499-2500, 1986.

$\left[\mathrm{JPT}^{+} 00\right]$ J. Kraus, P. Müller-Buschbaum, T. Kuhlmann, D. W. Schubert, and M. Stamm. Confinement effects on the chain conformation in thin polymer films. Europhysics Letters, 49(2):210-216, 2000.

[JSG ${ }^{+}$09] Y. K. Jhon, J. J. Semler, J. Genzer, M. Beevers, O. A. Gus'kova, P. G. Khalatur, and A. R. Khokhlov. Effect of comonomer sequence distribution on the adsorption of random copolymers onto impenetrable flat surfaces. Macromolecules, 42(7):2843-2853, 2009.

[KKK06] Y. A. Kriksin, P. G. Khalatur, and A. R. Khokhlov. Recognition of complex patterned substrates by heteropolymer chains consisting of multiple monomer articles. The Journal of Chemical Physics, 124(17):174904, 2006.

[Kle06] K. Kleiner. Assault on batteries. Nature, 441:1046-1047, 2006.

$\left[\mathrm{KLM}^{+}\right.$03] G. Karlström, R. Lindh, P.-Å. Malmqvist, B. O. Roos, U. Ryde, V. Veryazov, P.-O. Widmark, M. Cossi, B. Schimmelpfennig, P. Neogrady, and L. Seijo. Molcas: a program package for computational chemistry. Computational Materials Science, 28(2):222 - 239, 2003.

[KRS10] J. S. Kłos, D. Romeis, and J.-U. Sommer. Adsorption of random copolymers from a melt onto a solid surface: Monte carlo studies. The Journal of Chemical Physics, 132(2):024907, 2010.

[KVY88] S. K. Kumar, M. Vacatello, and D. Y. Yoon. Off-lattice monte carlo simulations of polymer melts confined between two plates. The Journal of Chemical Physics, 89(8):5206-5215, 1988.

[Lei80] L. Leibler. Theory of microphase separation in block copolymers. Macromolecules, 13(6):1602-1617, 1980.

[LMO $\left.{ }^{+} 09\right]$ C. Liang, T. Maruyama, Y. Ohmukai, T. Sotani, and H. Matsuyama. Characterization of random and multiblock copolymers of highly sul- 
fonated poly(arylene ether sulfone) for a proton-exchange membrane. Journal of Applied Polymer Science, 114(3):1793-1802, 2009.

[Lut88] J. F. Lutsko. Stress and elastic constants in anisotropic solids: Molecular dynamics techniques. Journal of Applied Physics, 64(3):1152-1154, 1988.

[Lut89] J. F. Lutsko. Generalized expressions for the calculation of elastic constants by computer simulation. Journal of Applied Physics, 65(8):29912997, 1989.

[Mat06] M. W. Matsen. Self-consistent field theory and its applications. In G. Gompper and M. Schick, editors, Soft Matter, Volume I: Polymer Melts and Mixtures, pages 87-178. Wiley-VCH, Weinheim, 2006.

[MBB01] C. Mischler, J. Baschnagel, and K. Binder. Polymer films in the normalliquid and supercooled state: a review of recent monte carlo simulation results. Advances in Colloid and Interface Science, 94(1-3):197-227, 2001.

$\left[\mathrm{MBO}^{+}\right.$09] Y. Meng, P. Bernazzani, P. A. O'Connell, G. B. McKenna, and S. L. Simon. A new pressurizable dilatometer for measuring the timedependent bulk modulus and pressure-volume-temperature properties of polymeric materials. Review of Scientific Instruments, 80(5):053903, 2009.

[MCH93] S. Melchionna, G. Ciccotti, and B. L. Holian. Hoover npt dynamics for systems varying in shape and size. Molecular Physics, 78(3):533-544, 1993.

[MGK99] M. Murat, G. S. Grest, and K. Kremer. Statics and dynamics of symmetric diblock copolymers: A molecular dynamics study. Macromolecules, 32(3):595-609, 1999.

[MJR ${ }^{+93]}$ A. M. Mayes, R. D. Johnson, T. P. Russell, S. D. Smith, S. K. Satija, and C. F. Majkrzak. Distributions of chain ends and junction points in ordered block copolymers. Macromolecules, 26(5):1047-1052, 1993. 


\section{Bibliography}

[MKS05] J. Mergheim, E. Kuhl, and P. Steinmann. A finite element method for the computational modelling of cohesive cracks. International Journal for Numerical Methods in Engineering, 63(2):276-289, 2005.

[MM96] S. T. Milner and D. C. Morse. Wetting description of block copolymer thin films. Physical Review E, 54(4):3793-3810, 1996.

[MP02] F. Müller-Plathe. Coarse-graining in polymer simulation: From the atomistic to the mesoscopic scale and back. ChemPhysChem, 3(9):754$769,2002$.

$\left[\mathrm{MRH}^{+}\right.$97] P. Mansky, T. P. Russell, C. J. Hawker, J. Mays, D. C. Cook, and S. K. Satija. Interfacial segregation in disordered block copolymers: Effect of tunable surface potentials. Physical Review Letters, 79:237-240, 1997.

$\left[\mathrm{MRR}^{+} 53\right]$ N. Metropolis, A. W. Rosenbluth, M. N. Rosenbluth, A. H. Teller, and E. Teller. Equation of state calculations by fast computing machines. The Journal of Chemical Physics, 21(6):1087-1092, 1953.

[MS94] M. W. Matsen and M. Schick. Stable and unstable phases of a diblock copolymer melt. Physical Review Letters, 72(16):2660-2663, 1994.

[MS05] M. Müller and G. D. Smith. Phase separation in binary mixtures containing polymers: A quantitative comparison of single-chain-in-meanfield simulations and computer simulations of the corresponding multichain systems. Journal of Polymer Science B Polymer Physics, 43:934958, 2005.

[MSD $\left.{ }^{+} 11\right]$ M. Müller, B. Steinmüller, K. C. Daoulas, A. Ramírez-Hernández, and J. J. de Pablo. Polymer-solid contacts described by soft, coarse-grained models. Physical Chemistry Chemical Physics, 13:10491-10502, 2011.

[NCH97] M. Nicodemi, A. Coniglio, and H. J. Herrmann. Frustration and slow dynamics of granular packings. Physical Review E, 55:3962-3969, 1997.

[NdlCC93] A. Nesarikar, M. Olvera de la Cruz, and B. Crist. Phase transitions in random copolymers. The Journal of Chemical Physics, 98(9):73857397, 1993. 
[NM02] J. R. Naughton and M. W. Matsen. Limitations of the dilution approximation for concentrated block copolymer/solvent mixtures. Macromolecules, 35(14):5688-5696, 2002.

[PBHK91] W. Paul, K. Binder, D. W. Heermann, and K. Kremer. Dynamics of polymer-solutions and melts - reptation predictions and scaling of relaxation-times. The Journal of Chemical Physics, 95:7726, 1991.

[PDS09] A. Polotsky, A. Degenhard, and F. Schmid. Random copolymer adsorption: Morita approximation compared to exact numerical simulations. The Journal of Chemical Physics, 131(5):054903, 2009.

[PdSK08] M. Praprotnik, L. delle Site, and K. Kremer. Multiscale simulation of soft matter: From scale bridging to adaptive resolution. Annual Review of Physical Chemistry, 59(1):545-571, 2008.

[Pli95] S. J. Plimpton. Fast parallel algorithms for short-range molecular dynamics. Journal of Computational Physics, 117:1-19, 1995.

[Rah64] A. Rahman. Correlations in the motion of atoms in liquid argon. Physical Review, 136(2A):A405-A411, 1964.

[Rap04] D. C. Rapaport. The Art of Molecular Dynamics Simulation. Cambridge University Press, Cambridge, 2nd edition, 2004.

[Ray88] J. R. Ray. Elastic constants and statistical ensembles in molecular dynamics. Computer Physics reports, 8(3):109-151, 1988.

[RC03] M. Rubinstein and R. H. Colby. Polymer Physics. Oxford University Press, Oxford, 2003.

[RDF78] P. J. Rossky, J. D. Doll, and H. L. Friedman. Brownian dynamics as smart monte-carlo simulation. The Journal of Chemical Physics, 69(10):4628-4633, 1978.

[RHDdP10] A. Ramírez-Hernández, F. A. Detcheverry, and J. J. de Pablo. Numerical simulation of gaussian chains near hard surfaces. The Journal of Chemical Physics, 133(6):064905, 2010. 


\section{Bibliography}

[RLM08] A. Roy, H.-S. Lee, and J. E. McGrath. Hydrophilic-hydrophobic multiblock copolymers based on poly(arylene ether sulfone)s as novel proton exchange membranes - part b. Polymer, 49(23):5037-5044, 2008.

[Roe94] R.-J. Roe. Short time dynamics of polymer liquid and glass studied by molecular dynamics simulation. The Journal of Chemical Physics, 100(2):1610-1619, 1994.

[SABW82] W. C. Swope, H. C. Andersen, P. H. Berens, and K. R. Wilson. A computer simulation method for the calculation of equilibrium constants for the formation of physical clusters of molecules: Application to small water clusters. The Journal of Chemical Physics, 76(1):637-649, 1982.

[SAK $\left.{ }^{+} 08\right]$ C. Shin, H. Ahn, E. Kim, D. Y. Ryu, J. Huh, K.-W. Kim, and T. P. Russell. Transition behavior of block copolymer thin films on preferential surfaces. Macromolecules, 41(23):9140-9145, 2008.

[SCB98] S. Srebnik, A. K. Chakraborty, and D. Bratko. Random heteropolymer adsorption on disordered multifunctional surfaces: Effect of specific intersegment interactions. The Journal of Chemical Physics, 109(15):6415-6419, 1998.

[SF79] J. M. H. M. Scheutjens and G. J. Fleer. Statistical theory of the adsorption of interacting chain molecules. 1. partition function, segment density distribution, and adsorption isotherms. The Journal of Physical Chemistry, 83(12):1619-1635, 1979.

[Sil82] A. Silberberg. Distribution of conformations and chain ends near the surface of a melt of linear flexible macromolecules. Journal of Colloid and Interface Science, 90(1):86-91, 1982.

[SMH $\left.{ }^{+} 11\right]$ B. Steinmüller, M. Müller, K. R. Hambrecht, G. D. Smith, and D. Bedrov. Properties of random block copolymer morphologies: Molecular dynamics and single-chain-in-mean-field simulations. $a c$ cepted by Macromolecules, 2011.

$\left[\mathrm{SMK}^{+}\right.$05] M. P. Stoykovich, M. Müller, S. O. Kim, H. H. Solak, E. W. Edwards, J. J. de Pablo, and P. F. Nealey. Directed assembly of block 
copolymer blends into nonregular device-oriented structures. Science, 308(5727):1442-1446, 2005.

[SND96] An-Chang Shi, Jaan Noolandi, and Rashmi C. Desai. Theory of anisotropic fluctuations in ordered block copolymer phases. Macromolecules, 29(20):6487-6504, 1996.

[SRB ${ }^{+}$07] T. D. Sewell, K. O. Rasmussen, D. Bedrov, G. D. Smith, and R. B. Thompson. Bidirectional mapping between self-consistent field theory and molecular dynamics: Application to immiscible homopolymer blends. The Journal of Chemical Physics, 127(14):144901, 2007.

[SS02] A. V. Subbotin and A. N. Semenov. Phase equilibria in random multiblock copolymers. The European Physical Journal E: Soft Matter and Biological Physics, 7:49-64, 2002.

[Sun98] H. Sun. Compass: An ab initio force-field optimized for condensedphase applicationsoverview with details on alkane and benzene compounds. The Journal of Physical Chemistry B, 102(38):7338-7364, 1998.

[SY05a] B. J. Sung and A. Yethiraj. Integral equation theory of random copolymer melts. Macromolecules, 38(5):2000-2008, 2005.

[SY05b] B. J. Sung and A. Yethiraj. Integral equation theory of random copolymer melts: Self-consistent treatment of intramolecular and intermolecular correlations. The Journal of Chemical Physics, 122(23):234904, 2005.

[SY05c] B. J. Sung and A. Yethiraj. Integral equation theory of randomly coupled multiblock copolymer melts: Effect of block size on the phase behavior. The Journal of Chemical Physics, 123(21):214901, 2005.

[TA01] Y. Tsori and D. Andelman. Diblock copolymer thin films: Parallel and perpendicular lamellar phases in the weak segregation limit. The European Physical Journal E: Soft Matter and Biological Physics, 5:605614, 2001. 10.1007/s101890170044. 


\section{Bibliography}

[TBM92] M. Tuckerman, B. J. Berne, and G. J. Martyna. Reversible multiple time scale molecular dynamics. The Journal of Chemical Physics, 97(3):1990-2001, 1992.

[TM05] C. A. Tyler and D. C. Morse. Orthorhombic $f d d d$ network in triblock and diblock copolymer melts. Physical Review Letters, 94:208302, 2005.

[TMBK97] M. E. Tuckerman, C. J. Mundy, S. Balasubramanian, and M. L. Klein. Modified nonequilibrium molecular dynamics for fluid flows with energy conservation. The Journal of Chemical Physics, 106(13):5615$5621,1997$.

[TWA ${ }^{+}$07] M. Takenaka, T. Wakada, S. Akasaka, S. Nishitsuji, K. Saijo, H. Shimizu, M. I. Kim, and H. Hasegawa. Orthorhombic fddd network in diblock copolymer melts. Macromolecules, 40(13):4399-4402, 2007.

[VB09] F. Varnik and K. Binder. Multiscale modeling of polymers at interfaces. International Journal of Materials Research, 2009/11:14941502, 2009.

[vdHMZ10] A. von der Heydt, M. Müller, and A. Zippelius. Sequence fractionation in symmetric random block copolymers. Macromolecules, 43:3161$3164,2010$.

[vdHMZ11] A. von der Heydt, M. Müller, and A. Zippelius. Three-phase coexistence with sequence partitioning in symmetric random block copolymers. Physical Review E, 83:051131, 2011.

[Ver67] L. Verlet. Computer "experiments" on classical fluids. i. thermodynamical properties of lennard-jones molecules. Physical Review, 159(1):98, 1967.

[vGB77] W. F. van Gunsteren and H. J. C. Berendsen. Algorithms for macromolecular dynamics and constraint dynamics. Molecular Physics: An International Journal at the Interface Between Chemistry and Physics, 34(5):1311-1327, 1977. 
[WBK $\left.{ }^{+} 11\right]$ H. Wagner, D. Bedorf, S. Küchemann, M. Schwabe, B. Zhang, W. Arnold, and K. Samwer. Local elastic properties of a metallic glass. Nature Materials, 10(6):439-442, 2011.

[WM09] J. Wang and M. Müller. Microphase separation of diblock copolymer brushes in selective solvents: Single-chain-in-mean-field simulations and integral geometry analysis. Macromolecules, 42(6):2251-2264, 2009.

[WSBM96] A. Werner, F. Schmid, K. Binder, and M. Müller. Diblock copolymers at a homopolymer-homopolymer interface: A monte carlo simulation. Macromolecules, 29(25):8241-8248, 1996.

[YDSN94] C. Yeung, R. C. Desai, A.-C. Shi, and J. Noolandi. Lower critical ordering temperature in diblock copolymer melts. Physical Review Letters, 72:1834-1837, 1994.

[YE77] S. C. Yeo and A. Eisenberg. Physical-properties and supermolecular structure of perfluorinated ion-containig (nafion) polymers. Journal of Applied Polymer Science, 21(4):875-898, 1977.

[YJvW $\left.{ }^{+} 04\right]$ K. Yoshimoto, T. S. Jain, K. van Workum, P. F. Nealey, and J. J. de Pablo. Mechanical heterogeneities in model polymer glasses at small length scales. Physical Review Letters, 93(17):175501, 2004.

[Yod98] R. Yoda. Elastomers for biomedical applications. Journal of Biomaterials Science, Polymer Edition, 9(6):561-626, 1998.

[YPLdP05] K. Yoshimoto, G. J. Papakonstantopoulos, J. F. Lutsko, and J. J. de Pablo. Statistical calculation of elastic moduli for atomistic models. Physical Review B, 71(18):184108, 2005.

[YW71] O. Yano and Y. Wada. Dynamic mechanical and dielectric relaxations of polystyrene below the glass temperature. Journal of Polymer Science Part A-2: Polymer Physics, 9(4):669-686, 1971.

[ZQZ $\left.{ }^{+} 10\right]$ G. Zhang, F. Qiu, H. Zhang, Y. Yang, and A.-C. Shi. Scft study of tiling patterns in abc star terpolymers. Macromolecules, 43(6):2981-2989, 2010. 
Bibliography

$\left[\mathrm{ZSVB}^{+}\right.$06] E. Zaccarelli, I. Saika-Voivod, S. V. Buldyrev, A. J. Moreno, P. Tartaglia, and F. Sciortino. Gel to glass transition in simulation of a valence-limited colloidal system. The Journal of Chemical Physics, 124(12):124908, 2006. 


\section{Liste der Veröffentlichungen}

1. B. Steinmüller, S. Leupold, ,Weighted finite energy sum rules for the omega meson in nuclear matter", Nuclear Physics A 778(3-4):195-216, 2006

2. B. Steinmüller, „Eine neue Initialisierung des Atomkerns für GiBUU“, Diplomarbeit, 2007

3. M. Müller, B. Steinmüller, K. C. Daoulas, A. Ramírez-Hernández, and J. J. de Pablo. „Polymer-solid contacts described by soft, coarse-grained models“, Physical Chemistry Chemical Physics, 13:10491-10502, 2011

4. B. Steinmüller, M. Müller, K. R. Hambrecht, G. D. Smith, and D. Bedrov. „Properties of random block copolymer morphologies: Molecular dynamics and Single-Chain-in-Mean-Field simulations“, accepted by Macromolecules, doi:10.1021/ma202311e, 2011 